\title{
GEOTRACES intercalibration of ${ }^{230} \mathrm{Th},{ }^{232} \mathrm{Th},{ }^{231} \mathrm{~Pa}$, and prospects for ${ }^{10} \mathrm{Be}$
}

\author{
Robert F. Anderson * , Martin Q. Fleisher ${ }^{1}$, Laura F. Robinson², R. Lawrence Edwards ${ }^{3}$, John A. Hoff ${ }^{3}$, S. Bradley \\ Moran ${ }^{4}$, Michiel Rutgers van der Loeff, Alexander L. Thomas 6 , Matthieu Roy-Barman 7 , and Roger Francois ${ }^{8}$ \\ ${ }^{1}$ Lamont-Doherty Earth Observatory of Columbia University, P.O. Box 1000, Palisades, NY 10964 USA \\ ${ }^{2}$ Department of Marine Chemistry and Geochemistry, Woods Hole Oceanographic Institution, Woods Hole, MA 02543 USA \\ and Department of Earth Sciences, University of Bristol, Bristol, UK \\ ${ }^{3}$ Department of Geology and Geophysics, University of Minnesota, Minneapolis, MN 55455 \\ ${ }^{4}$ Graduate School of Oceanography, University of Rhode Island, Narragansett, RI 02882-1197 USA \\ ${ }^{5}$ Alfred Wegener Institute for Polar and Marine Research, P.O. Box 120161, D-27515 Bremerhaven, Germany \\ ${ }^{6}$ Department of Earth Sciences, University of Oxford, South Parks Road, Oxford, OX1 3AN, UK \\ ${ }^{7}$ LSCE/IPSL Laboratoire CNRS/CEA/UVSQ, Domaine du CNRS, Avenue de la Terrasse, 91198 Gif-sur-Yvette Cedex, France \\ ${ }^{8}$ Department of Earth and Ocean Sciences, University of British Columbia, 6339 Stores Road, Vancouver, BC Canada V6T 1Z4
}

\begin{abstract}
Nineteen labs representing nine nations participated in the GEOTRACES intercalibration initiative that determined concentrations of ${ }^{232} \mathrm{Th},{ }^{230} \mathrm{Th},{ }^{231} \mathrm{~Pa}$, or ${ }^{10} \mathrm{Be}$ in seawater, suspended particles or sediments. Results generally demonstrated good agreement among labs that analyzed marine sediments. Two sets of seawater samples, aliquots of particulate material filtered in situ, and/or aliquots of biogenic sediments were distributed to participating labs. Internal consistency among participating labs improved substantially between the first and second set of seawater samples. Contamination was a serious problem for ${ }^{232} \mathrm{Th}$. Standard Niskin bottles introduced no detectable contamination, whereas sample containers, reagents, and labware were implicated as sources of contamination. No detectable differences in concentrations of dissolved ${ }^{232} \mathrm{Th},{ }^{230} \mathrm{Th}$, or ${ }^{231} \mathrm{~Pa}$ were observed among samples of seawater filtered through Nuclepore, Supor, or QMA (quartz) filters with pore diameters ranging between 0.4 and $1.0 \mu \mathrm{m}$. Isotope yield monitors equilibrate with dissolved Th in seawater on a time scale of much less than 1 day. Samples of filtered seawater acidified to a $\mathrm{pH}$ between 1.7 and 1.8 experienced no detectable loss of dissolved Th or Pa during storage for up to 3 years. The Bermuda Atlantic Time Series station will serve as a GEOTRACES baseline station for future intercalibration of ${ }^{232} \mathrm{Th}$ and ${ }^{230} \mathrm{Th}$ concentrations in seawater. Efforts to improve blanks and standard calibration are ongoing, as is the development of methods to determine concentrations of particulate nuclides, tests of different filtration methods, and an increasing awareness of the need to define protocols for reporting uncertainties.
\end{abstract}

Distributions of naturally occurring long-lived radionuclides provide quantitative information about the rates at which processes occur in the modern ocean (Francois 2007; Rutgers Van Der Loeff and Geibert 2008) while also providing insight into changes that have occurred in the past (Francois 2007; Henderson and Anderson 2003). As a consequence of their widespread use as tracers in ocean research, certain longlived radionuclides $\left({ }^{230} \mathrm{Th},{ }^{231} \mathrm{~Pa}\right)$ have been designated in the GEOTRACES Science Plan as "key" variables to be measured on all GEOTRACES ocean sections, whereas others $\left({ }^{232} \mathrm{Th},{ }^{10} \mathrm{Be}\right)$

*Corresponding author: E-mail: boba@Ideo.columbia.edu; phone: +1-845-365-8508

DOI 10.4319/lom.2012.10.179 are recommended to be measured where possible. Anticipating that concentrations of these nuclides will be determined by many labs worldwide throughout the duration of the GEOTRACES program, a substantial international intercalibration effort was organized to ensure accuracy and internal consistency throughout the global data set.

Analytical advances over the time span of a single generation have revolutionized the study of these radionuclides. The first reported measurements of dissolved ${ }^{230} \mathrm{Th}$ and ${ }^{231} \mathrm{~Pa}$ in open-ocean seawater involved the shipboard processing of sample sizes between 130 and 190 L. A volume this large was required to collect sufficient activity to determine nuclide concentrations by $\alpha$ decay counting techniques (Moore and Sackett 1964). Decay-counting methods remained the norm 
for the next two decades. Samples as large as $250 \mathrm{~L}$ were collected to study these nuclides (Nozaki et al. 1981; Nozaki and Nakanishi 1985). As an alternative to shipboard processing of large volumes of seawater, $\mathrm{MnO}_{2}$-impregnated absorbers were also employed to extract $\mathrm{Th}$ and $\mathrm{Pa}$ in situ (Anderson et al. 1983a, 1983b; Nozaki and Horibe 1983).

The transition from decay counting to the counting of atoms by mass spectrometry revolutionized the field, lowering sample size requirements by two orders of magnitude or more depending on the nuclide (Chen et al. 1986). Many of the labs participating in this intercalibration can now measure concentrations of ${ }^{230} \mathrm{Th}$ and ${ }^{232} \mathrm{Th}$ reliably using sample volumes of 1 to $2 \mathrm{~L}$ seawater. Some labs employ thermal ionization mass spectrometry (TIMS, e.g., Chen et al. 1986; Huh and Beasley 1987; Shen et al. 2003) whereas others use high-resolution inductively-coupled plasma mass spectrometry (ICP-MS, e.g., Choi et al. 2001; Fleisher and Anderson 2003; Shen et al. 2002). This substantial reduction in sample size represents a critical advancement that permits these nuclides to be measured routinely on GEOTRACES sections.

Although these nuclides have been studied for decades, procedures for collecting and processing large numbers of samples were relatively new and untested before GEOTRACES. A systematic intercalibration among a large number of labs had not been conducted previously, leaving some concern about the accuracy and internal consistency of prior results. Because all labs use isotope dilution, variable chemical recovery was not anticipated to be a problem. Furthermore, there is little industrial use of Th, and no industrial use of $\mathrm{Pa}$ or of ${ }^{10} \mathrm{Be}$, so contamination was also not expected to be a problem. Factors that were considered to be of potential concern included the following:

1) Calibration of chemical yield monitors (spike isotopes): The absence of readily available and universally accepted standards for the determination of these nuclides led to different strategies among participating labs for the calibration of chemical yield monitors (spikes). These strategies had not been compared systematically before the GEOTRACES initiative.

2) Sensitivity to filtration method: Thorium isotopes, ${ }^{231} \mathrm{~Pa}$, and ${ }^{10} \mathrm{Be}$ are highly insoluble in seawater. Thus, there was concern for loss of dissolved nuclides through sorption to filter material or to the filtration apparatus when determining dissolved nuclide concentrations.

3) Sample storage: Loss of these dissolved nuclides by precipitation or by sorption to container walls is another potential negative bias created by the insoluble nature of these nuclides. Previous studies have shown that dissolved Th and $\mathrm{Pa}$ are lost during storage of unacidified samples (Choi et al. 2001), and anecdotal accounts had suggested that dissolved Th could be lost over time even from acidified samples. Spiking samples at sea immediately following their collection would circumvent this problem. However, some nations impose strict restrictions on the use of artificial radionuclides aboard research vessels. Furthermore, the iso- tope of $\mathrm{Pa}$ used as a yield monitor $\left({ }^{233} \mathrm{~Pa}\right)$ has a half-life of only $27 \mathrm{~d}$. It is simply impractical to prepare a yield monitor before sailing, and then collect and process a large number of samples before ${ }^{233} \mathrm{~Pa}$ concentrations fall below detection limits. Storage of unspiked samples would be preferable provided this did not bias the results.

Perhaps the greatest surprise discovered during this intercalibration was the severity of the contamination problems that plagued the determination of ${ }^{232}$ Th concentrations in seawater. The foremost outcome of this study is to identify the critical nature of precautions to avoid contamination of seawater samples by extraneous sources of ${ }^{232} \mathrm{Th}$, and the importance of collecting blanks regularly during research cruises. By contrast, no serious problems were identified pertaining to sample storage or to different types of filter material, although additional tests of filtration methods constitute one of the recommendations for future work.

\section{Materials and procedures}

\section{Solicitation of participants}

Upon completion of the Science Plan in 2006, the GEOTRACES community turned its attention to intercalibration of methods as the next step in implementing the program. General information about the intercalibration process, together with a solicitation for participation by interested investigators, was distributed via the international GEOTRACES email list in July 2006, January 2007, and again in August 2007. Information about intercalibration was also posted on the GEOTRACES web site along with a list of all investigators interested in participating and the variable(s) that each investigator proposed to measure.

\section{Discussion of issues}

In July 2007, the authors of this paper contacted those investigators who had expressed an interest in participating in the intercalibration of ${ }^{230} \mathrm{Th},{ }^{232} \mathrm{Th},{ }^{231} \mathrm{~Pa}$, and/or ${ }^{10} \mathrm{Be}$. Investigators were asked to identify the nuclide(s) to be measured as well as the type(s) of samples to be analyzed (e.g., seawater, sediments, particles). Investigators were also polled about specific tests of sampling and analytical methods that could be made by the authors. Principal concerns that needed to be addressed before the international community could embark on a global study of these nuclides include the following.

1) Sample collection: With some exceptions (Moran et al. 1997, 2001, 2002), seawater samples for these nuclides have generally been collected using Niskin bottles or similar devices that are not specifically designed to avoid trace metal contamination. Tests had never been performed to compare Niskin bottles against trace metal-clean sampling systems. Consequently, intercalibration protocols were designed to allow a comparison between results obtained using Niskin bottles and results obtained using GO-Flo bottles (designed for trace metal sampling) as a byproduct of other tests described below.

2) Sample filtration: Filtration of seawater is necessary to determine operationally defined concentrations of dissolved 
nuclides, but no convenient or universally accepted procedure had been developed for samples as large as those required here (5 to $20 \mathrm{~L}$ ). Before GEOTRACES some labs had filtered seawater by gravity flow directly from Niskin bottles (e.g., Choi et al. 2001) whereas others transferred seawater to secondary containers for pressure filtration. Where GO-Flo bottles have been used previously to sample for these nuclides, seawater was filtered by pressurizing sample bottles with nitrogen (Moran et al. 1997, 2001, 2002). Still other investigators had limited their measurements to total (unfiltered) nuclide concentrations (e.g., Chase et al. 2003). Furthermore, there was no consensus about the best type of filter membrane to use. Following recommendations from the participants, the authors designed a system (described below in "Seawater samples and blanks") that allowed seawater to be pressure filtered directly from Niskin bottles. Pressure filtration was then used to test a variety of filter holders and filter membranes.

3) Processing at sea versus sample storage: Whether or not to spike and concentrate samples at sea was the topic that generated the most discussion. The principal argument in favor of processing samples at sea, at least through concentration by co-precipitation, is the desire to reduce the volume and weight of samples that must be shipped back to the lab (3000 to 5000 L water following a typical section cruise). Several concerns and arguments against processing at sea were discussed as well. For example, different labs use different carriers $\left[\mathrm{Fe}(\mathrm{OH})_{3}\right.$, $\left.\mathrm{MnO}_{2}, \mathrm{Mg}(\mathrm{OH})_{2}\right]$ to extract the nuclides from seawater. Furthermore, processing at sea requires the addition of ${ }^{229} \mathrm{Th}$ and ${ }^{233} \mathrm{~Pa}$ yield monitors. As noted above, some nations impose severe restrictions on the use of artificial radionuclides aboard research vessels, and some participants would not be allowed to use ${ }^{233} \mathrm{~Pa}$ on their cruises. All participants were concerned about the time frame for completion of sample analysis. Some cruises have a duration as much as two half lives of ${ }^{233} \mathrm{~Pa}$. More important, it is simply not possible to process hundreds of samples before the ${ }^{233} \mathrm{~Pa}$ has decayed below routine limits of detection. The option of $\gamma$ counting samples at sea after concentration by coprecipitation was considered as a strategy to determine the chemical yield of $\mathrm{Pa}$ before decay of ${ }^{233} \mathrm{~Pa}$. Following decay of the initial ${ }^{233} \mathrm{~Pa}$, the oxyhydroxide carrier could then be spiked with a second aliquot of ${ }^{233} \mathrm{~Pa}$ before completion of the analysis. Although processing of samples at sea was tested, in the end this proved to be a nonviable option because GEOTRACES cruises are so heavily subscribed that a sufficient number of berths are not available to accommodate the personnel who would be needed to collect and process the samples at sea. Consequently, samples for these nuclides collected during GEOTRACES cruises must be stored for processing in shore-based laboratories.

A rigorous comparison of replicate samples stored for variable periods of time had never been carried out. Choi et al. (2001) reported that dissolved $\mathrm{Th}$ and $\mathrm{Pa}$ were lost from unacidified samples during storage, and that external reproducibility was degraded following storage of acidified but unfiltered samples. Here, testing the quality of results obtained following storage of filtered and acidified samples was set as a high priority.

4) Particulate nuclide concentrations: A complete study of the marine biogeochemical cycles of ${ }^{230} \mathrm{Th},{ }^{232} \mathrm{Th}$, and ${ }^{231} \mathrm{~Pa}$ requires the collection and analysis of samples for their particulate as well as their dissolved concentrations. Tests for particulate ${ }^{10} \mathrm{Be}$ were not conducted because it was anticipated that concentrations of particulate ${ }^{10} \mathrm{Be}$ would be below detection limits given the partition coefficients reported by Chase et al. $(2002,2003)$ and the expected dissolved ${ }^{10} \mathrm{Be}$ concentrations. Based on the detection limits reported by many labs, it was estimated that a minimum of 100 to $150 \mathrm{~L}$ seawater must be filtered to measure concentrations of particulate ${ }^{231} \mathrm{~Pa}$. Samples of this size require in situ filtration, which was the purview of another intercalibration group led by investigators at the Woods Hole Oceanographic Institution (WHOI). Consequently, an agreement was negotiated with those investigators to provide filter material collected by in situ filtration for this intercalibration (see "Collection of particles" below). In addition, a large amount of siliceous ooze from the Southern Ocean was homogenized and distributed to participants to serve as a proxy for biogenic-rich particulate matter. Distribution of sediment samples also allowed for the participation by several labs that routinely analyze sediment samples, but which do not analyze seawater for these nuclides.

\section{Intercalibration samples}

Seawater and particulate material were collected during two intercalibration cruises aboard the RV Knorr: KN193-6, leg 2 (Bermuda to Norfolk, Virginia; July 2008) and KN195-8 (Honolulu, Hawaii to San Diego, California; May 2009).

\section{Collection of seawater}

Two rosette systems were used to collect seawater on each cruise: a standard rosette with 24 12-L Niskin bottles and the U.S. GEOTRACES sampling system (Cutter and Bruland 2012), which operates 24 12-L GO-Flo bottles. In addition, near-surface seawater was collected using the "GeoFish" towed sampling system designed at the University of California Santa Cruz.

The standard Niskin rosette was owned by the ship operators (WHOI) and its operation during these cruises followed standard ship protocols, including the use of steel hydrowire and closing of bottles on the up cast of the package. Niskin bottles were equipped with nylon-coated closure springs and Viton O-rings. When the Niskin rosette was used to test different filtration methods, all 24 bottles were closed at a single depth to provide replicate samples that were assumed to be from a homogeneous water mass. Typically, water from each Niskin bottle was filtered through a single filter, except in a few cases where it was necessary to change filters that had become clogged. The ship's rosette was also used to produce full water column profiles of the concentrations of ${ }^{230} \mathrm{Th}$, ${ }^{232} \mathrm{Th}$, and ${ }^{231} \mathrm{~Pa}$. There, three Niskin bottles were fired at each of eight depths to provide replicate samples (see "Baseline station-BATS" discussing baseline profiles). 
Water to be distributed to the labs participating in this intercalibration was collected near the surface $(5$ to $15 \mathrm{~m})$ using the GeoFish and from mid depth using the U.S. GEOTRACES sampling system. During the first cruise (KN193-6, Leg 2) mid-depth water was collected from $2000 \mathrm{~m}$ at the Bermuda Atlantic Time Series Station (BATS; roughly $75 \mathrm{~km}$ southeast of Bermuda at $\left.31^{\circ} 50^{\prime} \mathrm{N}, 64^{\circ} 10^{\prime} \mathrm{W}\right)$. Surface water was also collected near the BATS site. Similar protocols were followed during the second cruise (KN195-8), where middepth water was collected from $3000 \mathrm{~m}$ at a station located at $30^{\circ} \mathrm{N}$ and $140^{\circ} \mathrm{W}$ (also known as the SAFe station). At each location, five casts of the U.S. GEOTRACES system were used to collect $\sim 1000 \mathrm{~L}$ of mid-depth seawater for this intercalibration.

Water from GO-Flo bottles and from the fish was filtered through 10-inch Osmonics (Memtrex) cartridges containing sequential $0.45 \mu \mathrm{m}$ then $0.2 \mu \mathrm{m}$ poresize polycarbonate, track etched (PCTE) pleated filter membrane, with a total filtration surface area of 1.6 square meters. All filtrations were performed inside a portable clean van. Filtered water was pumped into two $500 \mathrm{~L}$ acid-cleaned fluorinated low density polyethylene tanks through PFA Teflon tubing using an all PFA Teflon diaphragm pump. The two tanks were connected with all Teflon tubing, valves, and fittings. Water was pumped continuously between the two 500-L tanks to produce and maintain a homogenized sample. Once the tanks were filled, the water was acidified to $\mathrm{pH} \sim 1.7$ using either Fisher Optima $\mathrm{HCl}$, Seastar $\mathrm{HCl}$, laboratory redistilled $\mathrm{HCl}$ or a combination of these sources. Individual aliquots of 1 to $20 \mathrm{~L}$ were drawn from the filtered, acidified, and homogenized seawater for distribution to participating labs.

Each participating lab provided its own precleaned sample containers, which were shipped to the cruise and back to the home institution inside double plastic bags to reduce the chance of contamination. Each lab was normally provided with three 10-L samples from each deep intercalibration depth and a single 10-L sample from the near-surface depth. Triplicate samples from the deeper intercalibration depth were intended to provide a measure of internal reproducibility. Some labs subdivided their 10 -L samples to provide a larger number of replicates.

\section{Biogenic sediments}

Silicious ooze sediment was collected by the LDEO authors in 1996 during a cruise of the RVIB Nathaniel B. Palmer (NBP9604 Station 3, Lat $61.95^{\circ} \mathrm{S}$ Lon $170.05^{\circ} \mathrm{W}$, water depth $3404 \mathrm{~m})$. Approximately $120 \mathrm{~g}$ dry weight of near-surface sediment recovered from the trimmings of extruded multi-cores was homogenized, freeze-dried, and distributed to participating labs. The sediment had an opal content of $\sim 71 \%$ and a $\mathrm{CaCO}_{3}$ content of $\sim 7 \%$.

\section{Collection of particles:}

To provide a sufficient mass of particulate material for the measurement of ${ }^{230} \mathrm{Th}$ and ${ }^{231} \mathrm{~Pa}$, and at the same time, obtain comparable samples from the same depth and time, investiga- tors at WHOI designed and built a trace metal clean pumprosette (Maiti et al. 2012). The rosette frame was made of epoxy-coated aluminum and deployed from a Kevlar wire. Up to eight battery-powered McLane pumps were operated simultaneously at a single depth, effectively providing samples of particulate material from the same water mass.

A PVC device was designed to subsample filters. For subsampling, a filter was placed on a base plate constructed of the same frit material as used in the McLane pump filter-holders. The base of the device was attached to a vacuum pump to hold the filters in place during subsampling, and the filters were sliced wet. A slotted PVC ring placed around the outside the filter allowed each filter to be subdivided into halves, quarters, or eighths with a ceramic knife. The base frit was replaced or acid washed between each set of filters. All processing was carried out under clean conditions in a high efficiency particulate air (HEPA) flow bench. Under shore-based lab conditions blank filters were sliced and weighed and the variability in weight between quarters was found to be less than $2 \%$, providing a measure of subsampling reproducibility. Subsamples from a single sample filter counted for ${ }^{234} \mathrm{Th}$ indicated intrafilter variability of $\sim 2.5 \%$, whereas differences between subsamples from filters housed in different pumps on the same rosette cast exhibited variability of between $15 \%$ and $20 \%$ (Maiti et al. 2012). Ultimately, this interpump variability places a practical limit on our ability to intercalibrate the determination of concentrations of particulate ${ }^{230} \mathrm{Th},{ }^{232} \mathrm{Th}$, and ${ }^{231} \mathrm{~Pa}$.

Particles were collected for the Th-Pa intercalibration using $142 \mathrm{~mm}$ diameter Supor polyethersulfone filters $(0.45 \mu \mathrm{m}$ pore diameter). During a cast each pump generally filtered between 400 and 600 L. Quarter-filters, sectioned as described above, were provided by investigators at WHOI and distributed by the authors to participating labs.

A blank filter (dip blank) for each McLane pump was produced by inserting an individual 142-mm Supor filter into a $53 \mu \mathrm{m}$ mesh bag (closed on all sides) and then placing the bag in a plastic box with holes drilled through its sides. Each pump had its own dip-blank box attached to its frame. Filters used for dip blanks were taken from the same batch as those used for the intercalibration samples. Dip-blank filters were sectioned following the same protocol that was used for the sample filters. Dip-blank filters were analyzed by participating labs to estimate the total procedural blank for particulate analysis. Although the dip blank filters do not provide a true procedural blank, it is thought that they provide a more accurate representation of the particulate blank than would be offered by a reagent blank alone.

\section{Participating labs}

A summary of the laboratories that submitted results for this intercalibration is provided in Table 1. Also reported are the variables reported by each lab as well as a summary of the principal features of the analytical procedures employed by each lab. 
Table 1. Labs participating in the $\mathrm{Th}, \mathrm{Pa}, \mathrm{Be}$ intercalibration.

\begin{tabular}{|c|c|c|c|c|c|c|}
\hline Principal investigator & Lab & Nation & $\begin{array}{c}\text { Sediments } \\
\text { Particles }\end{array}$ & $\begin{array}{c}\text { Diss. } \\
\text { Th, Pa }\end{array}$ & ${ }^{10} \mathrm{Be}$ & Methods \\
\hline Anderson, R. & LDEO & USA & $x$ & $x$ & & $1 \mathrm{a}, 2 \mathrm{a}, 3 \mathrm{~b}$ \\
\hline Andersson, $\mathrm{P}$. & LIG/NRM & Sweden & $x$ & $x$ & & $1 a, 2 b, 2 c, 3 a$ \\
\hline Chase, Z. & Oregon St. U & USA & $x$ & & & $2 a, 3 b$ \\
\hline Edwards, RL/Moran SB & Minnesota/Rhode Island & USA & $x$ & $x$ & & $1 a, 2 a, 3 c$ \\
\hline Francois, R. & U British Columbia & Canada & $x$ & $x$ & & $1 a, 2 a, 3 b$ \\
\hline Geibert, W. & U Edinburgh & UK & & & $x$ & $1 a, 2 a, 2 d, 3 d$ \\
\hline Frank, M. & IFM-GEOMAR & Germany & & & $x$ & $1 \mathrm{a}, 2 \mathrm{a}, 2 \mathrm{e}, 3 \mathrm{~d}$ \\
\hline Henderson, G. & Oxford U & UK & $x$ & $x$ & & $1 a, 2 a, 3 c$ \\
\hline Hong, G. & KORDI & Korea & $x$ & & & $2 a, 3 b$ \\
\hline Jeandel, C. & LEGOS & France & $x$ & $x$ & & $1 \mathrm{a}, 2 \mathrm{a}, 3 \mathrm{c}$ \\
\hline Marcantonio, F. & Texas A\&M U & USA & $x$ & $x$ & & $1 \mathrm{a}, 2 \mathrm{a}, 3 \mathrm{~b}$ \\
\hline Nagai, $\mathrm{H}$. & Nihon U & Japan & & & $x$ & $1 \mathrm{a}, 2 \mathrm{a}, 2 \mathrm{~d}, 3 \mathrm{~d}$ \\
\hline Okubo, A. & U Tokyo & Japan & $x$ & $x$ & & $1 a, 2 a, 3 b$ \\
\hline Pichat, S. & ENS-Lyon & France & $x$ & & & $2 a, 3 c$ \\
\hline Robinson, L. & WHOI & USA & $x$ & $x$ & & $1 a, 2 a, 3 c$ \\
\hline Roy-Barman, M. & LSCE & France & & $x$ & & $1 a, 2 a, 3 a, 3 c$ \\
\hline Rutgers vd Loeff, M. & AWI & Germany & $x$ & $x$ & & $1 \mathrm{a}, 2 \mathrm{a}, 3 \mathrm{~b}$ \\
\hline Scholten, J. & IAEA & Monaco & $x$ & $x$ & & $1 c, 2 a, 3 c$ \\
\hline Yamada, Y. & NIRS & Japan & $x$ & & & $2 a, 3 b$ \\
\hline
\end{tabular}

Preconcentration: $1 \mathrm{a}, \mathrm{Fe}(\mathrm{OH})_{3} ; 1 \mathrm{~b}, \mathrm{MnO}_{2} ; 1 \mathrm{c}, \mathrm{Mg}(\mathrm{OH})_{2}$

Purification: 2a, AG1 $\times 8$; 2b, UTEVA Eichrom; 2c, TRU Eichrom; 2d, AG50 × 8; 2e, Coprecipitation in the presence of EDTA

Detection: 3a, TIMS; 3b, SC-ICP-MS; 3c, MC-ICP-MS; 3d, accelerator mass spectrometry

\section{Procedures used at LDEO}

The LDEO authors were responsible for a number of tests recommended by the participants (See "Discussion of issues"). Therefore, the methods employed at LDEO are described here in some detail.

\section{Seawater samples and blanks}

Seawater samples were collected in low-density polyethylene cubitainers (either 1 gallon or $10 \mathrm{~L}$ volume; Hedwin Corp.) that had been cleaned by soaking in $10 \% \mathrm{HCl}$ (trace metal grade, Fisher Scientific) followed by Milli-Q water rinse. Blanks were prepared at sea by adding at least 2 L Milli-Q water to a cubitainer and then treating it as a sample.

Niskin bottles were sampled on the open deck of the ship while still in place on the rosette. Sampling under these conditions presented a potential source of contamination, but no alternative was available. Sampling was completed as rapidly as possible, and the sampling/filtration system was designed to minimize the potential for contamination from the shipboard environment. For unfiltered samples, water was drained via acid washed Teflon-lined Tygon tubing directly into the cubitainers. Filtered samples were collected by in-line filtration from pressurized Niskin bottles. Bottles were pressurized at 8-10 psi (54-70 kPa) with a system that distributed filtered air from a compressor via a manifold to each bottle through a fitting placed in its air vent. Each distribution line from the manifold was fitted with a valve so that each bottle could be pressurized or vented independently, while still allowing for the possibility to simultaneously filter all the bottles. Pressurized bottles were held closed with Irwin Quick-Grip bar clamps spanning the endcaps. No attempt was made to evaluate the blank contributed by the pressurization scheme. However, pressurization of Niskin bottles has been discontinued on subsequent U.S. GEOTRACES cruises in favor of gravity filtration through Acropak capsules.

The various filter types were precleaned in batches by soaking for several days in $10 \% \mathrm{HCl}$ (trace metal grade, Fisher Scientific), followed by repeated soaking in Milli-Q water until a neutral $\mathrm{pH}$ was measured. All of the shipboard filter handling, including loading into Savillex filter holders (47 $\mathrm{mm}$ or $90 \mathrm{~mm}$ ) was carried out inside a HEPA-filtered laminar flow bench located within the main lab of the ship. Filter holders were rinsed with Milli-Q water between each use. Teflon-lined Tygon tubing connected the filter holders to the petcocks of the Niskin bottles. Each filter holder was held in place directly above the mouth of the cubitainer, so that filtered water flowed directly into the cubitainer. The first few milliliters of filtrate were used to rinse the cubitainer and then discarded.

SAFe tanks were located inside an enclosure constructed with plastic sheeting held in place by a wooden frame. Water from the SAFe tanks was transferred to each cubitainer through a length of plastic tubing connected to the Teflon 
diaphragm that circulated water between the tanks. Sample containers (both those from LDEO and those provided by participating labs) were rinsed with water from the tank before filling. Labs participating in the intercalibration generally provided their own sample containers, each cleaned by the protocol normally used by the lab. Labs that requested samples after the completion of the first cruise were provided with seawater collected in cubitainers cleaned at LDEO and archived for future use.

Samples from the Niskin bottles were acidified to between pH 1.7 and 1.8 using Optima $\mathrm{HCl}(\sim 40 \mathrm{~mL}$ of 6 Normal $\mathrm{HCl}$ for a 10-L sample). All samples, whether collected from Niskin bottles or from the SAFe tank, were double bagged for shipment to their final destinations.

\section{Coprecipitation and digestion of seawater samples}

Unless otherwise noted, all of the acids and the ammonium hydroxide used at LDEO in the procedures outlined below were Optima Grade from Fisher Scientific.

1) At LDEO, each seawater sample was weighed (corrected for the tare weight of the cubitainer and for the weight of $6 \mathrm{~N}$ $\mathrm{HCl}$ added at sea), spiked with known (weighed) amounts of isotope yield monitors (about $20 \mathrm{pg}{ }^{229} \mathrm{Th}$ and $0.5 \mathrm{pg}$ ${ }^{233} \mathrm{~Pa}$ for each sample), and $100 \mu \mathrm{L}$ Fe carrier $\left(\mathrm{FeCl}_{3}, \sim 150\right.$ $\mathrm{mg} \mathrm{Fe} / \mathrm{mL}$, purified by repeated extraction into isopropyl ether) was added.

2) The $\mathrm{pH}$ was raised to between 8.0 and 8.5 with $\mathrm{NH}_{4} \mathrm{OH}$ to precipitate iron hydroxide, which was concentrated by settling and centrifugation before digestion in a sequence of $\mathrm{HNO}_{3}, \mathrm{HClO}_{4}$, and $\mathrm{HF}$. The $\mathrm{HClO}_{4}$ and $\mathrm{HF}$ eliminate organic matter and amorphous silicon that are carried by the iron hydroxide. All steps following centrifugation took place in a HEPA-filtered laminar bench.

3) The digested sample was taken up in dilute $\mathrm{HCl}$ and the iron hydroxide precipitation step was repeated.

4) The precipitate was dissolved in $\mathrm{HCl}$, heated to dryness at $120^{\circ} \mathrm{C}$, and dissolved in $1 \mathrm{~mL}$ concentrated $\mathrm{HCl}$ (heating at $50^{\circ} \mathrm{C}$ as needed to dissolve precipitate).

\section{Column chemistry}

Purification of Th and $\mathrm{Pa}$ was carried out by ion exchange using a series of three columns. All of the column chemistry was conducted using columns with a bed volume of $1 \mathrm{ml}$ BioRad AG1-X8 100-200 mesh anion resin ( $\mathrm{Cl}^{-}$form). Columns were polypropylene with $45 \mu \mathrm{m}$ porous polyethylene frits and a reservoir volume of $\sim 6 \mathrm{~mL}$.

\section{Step \#1 (Separation of Th and Pa)}

1) After preconditioning the column with concentrated $\mathrm{HCl}$, the sample was loaded onto the column in concentrated $\mathrm{HCl}(\sim 1 \mathrm{~mL}$, from above), which was collected along with $6 \mathrm{~mL}(4 \times 1.5 \mathrm{~mL}$ aliquots $)$ concentrated $\mathrm{HCl}$ rinse as the Th fraction.

2) Pa was eluted with $3 \times 2 \mathrm{ml}$ concentrated $\mathrm{HCl} / 0.13 \mathrm{~N} \mathrm{HF}$.

3) Iron was washed off the resin with $10-15 \mathrm{~mL}$ dilute $(\sim 1 \%)$ $\mathrm{HCl}$, and the column was held for Step 2 below.

4) The $\mathrm{Th}$ and $\mathrm{Pa}$ fractions were dried down overnight at $105^{\circ} \mathrm{C}$, after the addition of $0.5 \mathrm{~mL}$ of concentrated $\mathrm{HNO}_{3}$ and 2 drops of concentrated $\mathrm{HClO}_{4}$ to decompose any organics that eluted from the resin. Samples were heated to a small drop of $\mathrm{HClO}_{4}$. Step \#2 (Final purification of Th)

1) The Th fraction was converted to $\mathrm{HNO}_{3}$, heated to fuming $\mathrm{HClO}_{4}$, and taken up in $1 \mathrm{~mL} 8 \mathrm{~N} \mathrm{HNO}_{3}$, before loading onto the original ion exchange column that was reconditioned with $2 \times 2 \mathrm{~mL} 8 \mathrm{~N} \mathrm{HNO}_{3}$.

2) The beaker and column were rinsed with $4 \times 1 \mathrm{~mL} 8 \mathrm{~N}$ $\mathrm{HNO}_{3}$.

3) Th was eluted with $200 \mu \mathrm{L}$ concentrated $\mathrm{HCl}$, followed by $4 \times 1.5 \mathrm{~mL}$ concentrated $\mathrm{HCl}$.

4) Two drops of $\mathrm{HClO}_{4}$ and $0.5 \mathrm{~mL}$ concentrated $\mathrm{HNO}_{3}$ were added and the solution was dried overnight at $\sim 100^{\circ} \mathrm{C}$.

5) The following day, $250 \mu \mathrm{L}$ concentrated $\mathrm{HNO}_{3}$ (and a drop of $\mathrm{HClO}_{4}$ if the sample went dry overnight) were added and the solution was heated to a drop of fuming $\mathrm{HClO}_{4}$ at $180^{\circ} \mathrm{C}$. In preparation for analysis by ICP-MS, the sample was taken up in $1 \mathrm{~mL}$ of $1 \% \mathrm{HNO}_{3} / 0.1 \% \mathrm{HF}$, capped tightly and cooled. The small amount of HF was found to help reduce memory effects in the sample introduction system, including the Aridus desolvating nebulizer unit.

Step \#3 (Final purification of $\mathrm{Pa}$ )

1) A drop of $\mathrm{HClO}_{4}$ and $200 \mu \mathrm{L}$ concentrated $\mathrm{HNO}_{3}$ were added to the $\mathrm{Pa}$ fraction, which was heated at $180^{\circ} \mathrm{C}$ to fuming $\mathrm{HClO}_{4}$ to drive off all remaining $\mathrm{HF}$. The sample was taken up in a few drops of concentrated $\mathrm{HCl}$ and heated again to fuming $\mathrm{HClO}_{4}$ at $180^{\circ} \mathrm{C}$. Two drops of concentrated $\mathrm{HCl}$ were added and the beaker was cooled before adding $1 \mathrm{~mL}$ concentrated $\mathrm{HCl}$.

2) The column used for $\mathrm{Th}$ was reconditioned with $3 \mathrm{~mL}$ trace metal grade concentrated $\mathrm{HCl} / 0.13 \mathrm{~N} \mathrm{HF}, 2$ full reservoirs Milli-Q water, and finally $2 \times 2 \mathrm{~mL}$ concentrated Optima Grade $\mathrm{HCl}$.

3) The sample was loaded on the column in $1 \mathrm{~mL}$ concentrated $\mathrm{HCl}$, followed by six 1-mL concentrated $\mathrm{HCl}$ rinses, after which $\mathrm{Pa}$ was eluted with $3 \times 2 \mathrm{~mL}$ concentrated $\mathrm{HCl} / 0.13 \mathrm{~N} \mathrm{HF}$.

4) Two drops of $\mathrm{HClO}_{4}$ and $0.5 \mathrm{~mL}$ concentrated $\mathrm{HNO}_{3}$ were added and the solution was dried overnight at $\sim 100^{\circ} \mathrm{C}$.

5) Another drop of $\mathrm{HClO}_{4}$ and $200 \mu \mathrm{L}$ concentrated $\mathrm{HNO}_{3}$ were added as needed, the sample was dried at $100^{\circ} \mathrm{C}$ to a drop of $\mathrm{HClO}_{4}$ and take up in $0.75 \mathrm{~mL}$ of $1 \% \mathrm{HNO}_{3} / 0.1 \% \mathrm{HF}$.

\section{Filter digestion}

Supor polyethersulfone filters were used for the GEOTRACES intercalibration of particulate Th and $\mathrm{Pa}$ concentrations. Total digestion of these filters presented problems for several groups, so the method used at LDEO to successfully digest this filter material is described below.

\section{Lab equipment}

1) This procedure uses perchloric acid, so a hood certified for $\mathrm{HClO}_{4}$ use is absolutely required.

2) A hot plate capable of reaching $200-220^{\circ} \mathrm{C}$. 
3) $60 \mathrm{~mL}$ Savillex Teflon jars or equivalent.

4) Concave "White" Teflon watch glasses to fit the $60 \mathrm{~mL}$ Savillex jars.

\section{Laboratory procedure}

1) Weighed spikes, $100 \mu \mathrm{L}$ of Fe carrier (Section 3.2) and $5 \mathrm{~mL}$ concentrated $\mathrm{HNO}_{3}$ were added to the filter in a $60 \mathrm{~mL}$ Savillex jar.

2) The sample was heated overnight at $150^{\circ} \mathrm{C}$ in the Savillex jar covered with a white Teflon watch glass. $\mathrm{HNO}_{3}$ was added as needed to maintain volume.

3) The sample was allowed to cool, after which $3 \mathrm{~mL}$ concentrated $\mathrm{HClO}_{4}$ were added and the temperature was raised to $200^{\circ} \mathrm{C}$. The sample was heated uncovered until white $\mathrm{HClO}_{4}$ fumes appear.

4) The hotplate temperature was reduced to $180^{\circ} \mathrm{C}$, and the sample was covered again with the white Teflon watch glass. After about $30 \mathrm{~min}$, the $\mathrm{HClO}_{4}$ began to oxidize the Supor filter (as evidenced by foaming on the filter surface). Soon thereafter the oxidation reaction accelerated, sometimes becoming violent enough that some of the filter material splattered onto the watch glass and walls of the jar.

5) Upon completion of the oxidation, the walls and watch glass were rinsed with water into the jar, and the contents were heated to fuming $\mathrm{HClO}_{4}$ at $200^{\circ} \mathrm{C}$. If necessary, 1 or 2 $\mathrm{mL}$ additional $\mathrm{HClO}_{4}$ were added. About an hour was needed, depending on the amount of water and $\mathrm{HClO}_{4}$ added. Dissolution was complete when the solution was visibly free of any remaining filter material. Heating was continued until dense $\mathrm{HClO}_{4}$ fumes appeared.

6) The sample was allowed to cool briefly, after which 10 drops (200-300 $\mu \mathrm{L})$ concentrated HF were added to attack the silicate minerals and opal in the particles. The sample was heated until $\mathrm{HClO}_{4}$ fumes appeared and then an additional 5 drops of HF were added.

7) The sample was heated to the fuming $\mathrm{HClO}_{4}$ stage again, after which the jar walls were rinsed with concentrated $\mathrm{HNO}_{3}$.

8) Heating continued until less than $0.5 \mathrm{~mL}$ solution remained.

9) $0.5 \mathrm{~mL}$ concentrated $\mathrm{HCl}$ and then $5 \mathrm{~mL}$ water were added to the jar.

10) The sample was heated for a few minutes on the hot plate, then removed from the hot plate, covered, and cooled. The solution had a yellow/orange color from the iron, but it was free of visible solids.

At this point, the solution was transferred to a $50-\mathrm{mL}$ centrifuge tube with a few $5 \mathrm{~mL}$ water rinses of the sample jar. $\mathrm{NH}_{4} \mathrm{OH}$ was used to raise the $\mathrm{pH}$ to $\sim 8$ and iron hydroxide precipitated. After centrifuging, the $\mathrm{Fe}(\mathrm{OH})_{3}$ was washed in Milli$\mathrm{Q}$ water, centrifuged again, and dissolved in $\mathrm{HCl}$. From that point, the column chemistry was the same as that described above for seawater samples.

Note that step 4 involves a violent oxidation reaction. Test this procedure using a small piece of filter material before applying it to a complete sample.

\section{ICP-MS run information}

Analyses were made on a VG Elemental AXIOM Single Collector Magnetic Sector ICP-MS with a Resolving Power of $\sim 400$ to ensure the highest sensitivity. All measurements were done using a peak jumping routine in ion counting mode. A solution of SRM129, a natural U standard, was run to determine the mass bias correction (assuming that the mass fractionation for $\mathrm{Th}$ and $\mathrm{Pa}$ are the same as for $\mathrm{U}$ ). The concentration of $\mathrm{U}$ in the SRM129 solution was $100 \mathrm{pg} / \mathrm{mL}$.

An aliquot of the final Th solution, typically $100 \mu \mathrm{L}$, was diluted to $800 \mu \mathrm{L}$ with the LDEO "run solution" (1\% $\mathrm{HNO}_{3} / 0.1 \% \mathrm{HF}$ ) for the ${ }^{232} \mathrm{Th}$ measurement. The remaining $900 \mu \mathrm{L}$ of the final Th solution was used without further dilution for the ${ }^{230} \mathrm{Th}$ measurement. This scheme was more time consuming than measuring all of the Th isotopes at once, but it allowed ${ }^{232} \mathrm{Th}$ to be measured on the multiplier so there was no need to make a Faraday/multiplier gain correction for the ${ }^{232}$ Th analyses. SRM129 was run three times each day with both the $\mathrm{Th}$ and $\mathrm{Pa}$ analyses. Each sample measurement was bracketed by measurement of an aliquot of the run solution, used to correct for the instrument background count rates on the masses measured.

To correct for potential tailing of ${ }^{232} \mathrm{Th}$ into the minor Th and $\mathrm{Pa}$ isotopes, beam intensities were measured at the half masses above and below each mass for ${ }^{230} \mathrm{Th},{ }^{231} \mathrm{~Pa}$, and ${ }^{233} \mathrm{~Pa}$. Tailing under each minor isotope was estimated as the log mean intensity of the half masses on either side of each minor isotope.

For all results from LDEO, error bars represent the propagation of one sigma errors based on the standard deviation of five sequences of isotope ratios collected by ICP-MS, our estimated error in the ${ }^{229} \mathrm{Th}$ or ${ }^{233} \mathrm{~Pa}$ spike concentration, and the blank correction of the individual isotopes for each sample batch. Blanks processed concurrently with each batch of samples were used rather than a grand mean blank covering the entire intercalibration effort because blank levels were reduced significantly during the course of this work, especially for ${ }^{232} \mathrm{Th}$ (from $\sim 100 \mathrm{pg}$ initially to $\sim 10 \mathrm{pg}$ for the last samples processed). Blanks were reduced primarily by carrying out sample digestions and column chemistry inside a laminar flow bench. Further reduction of the blank was achieved by adopting a modified version of the procedure for cleaning anion exchange resin described by Andersson and Schöberg (2012). Acid cleaning the microcentrifuge tubes used as sample vials for the ICP-MS also helped reduce the blank.

Additional information about sampling and purification methods, as well as information about offline processing of ICP-MS data, is available on request from the LDEO authors.

\section{Developing a baseline station at BATS}

Two additional sets of results from the Bermuda Atlantic Time Series site were combined with those from the first GEOTRACES intercalibration cruise to establish a baseline station for future intercalibration of $\mathrm{Th}$ and $\mathrm{Pa}$ (see "Baseline station- 
BATS" below). One set of seawater samples collected before GEOTRACES was analyzed at the University of Minnesota. The other, collected subsequent to the GEOTRACES cruise, was processed at WHOI. Procedures employed in processing and analyzing the second set of samples are described in an accompanying paper (Auro et al. 2012). Methods used at the University of Minnesota are described below.

\section{Minnesota chemical procedures}

Two-liter seawater samples were collected in July 1993 and analyzed for ${ }^{230} \mathrm{Th}$ later that year. Chemical purification of Th for these samples was performed either at the U. Minnesota (by J. Hoff) or at WHOI (by K. Buesseler, R.A. Belastock, and S.B. Moran). Purified Th separates were all analyzed at the U. Minnesota.

Seawater samples were processed in clean rooms at WHOI or Minnesota using ultrapure reagents following similar separation procedures. Seawater samples were transferred on board the ship into acid-cleaned polyethylene bottles. Upon return to the lab, the samples were weighed, acidified by addition of $1 \mathrm{~mL}$ of concentrated $\mathrm{HCl}$, and spiked with $\sim 50 \mathrm{pg}$ of ${ }^{229} \mathrm{Th}$ tracer and $\sim 6 \mathrm{mg}$ of Fe carrier. The sample/spike mixture was gently heated $\left(\sim 75^{\circ} \mathrm{C}\right)$ for 2 or more days to ensure sample/spike equilibration. Thorium was coprecipitated with $\mathrm{Fe}(\mathrm{OH})_{3}$ by addition of $\mathrm{NH}_{4} \mathrm{OH}$ until the seawater solution reached a $\mathrm{pH}$ of 8 to 9 (Goldberg et al. 1963). The precipitate was allowed to settle for at least $1 \mathrm{~d}$ and then either vacuum filtered onto 0.45 $\mu \mathrm{m}$ Durapore filters (WHOI) or isolated using centrifugation (Minnesota). The filtrate was then dissolved in $\sim 1 \mathrm{~mL}$ of $8 \mathrm{~N} \mathrm{HNO}_{3}$ and transferred to a Teflon vial for separation chemistry. Chemical separation procedures for Th were modified from Chen et al. (1986) and have been summarized elsewhere (Moran et al. 1997). Two anion-exchange columns (BioRad AG 1-X8, 100-200 mesh) were used to separate $\mathrm{Th}$. The initial column contained an $800 \mu \mathrm{L}$ resin bed and the second column had a $175 \mu \mathrm{L}$ resin bed. For both columns, the same procedures were used, including preconditioning with 3 column volumes $(\mathrm{cv})$ of $6 \mathrm{~N} \mathrm{HCl}, 3 \mathrm{cv}$ of $\mathrm{H}_{2} \mathrm{O}$, and $3 \mathrm{cv}$ of $7 \mathrm{~N} \mathrm{HNO}_{3}$. Fe was eluted with $3 \mathrm{cv}$ of $7 \mathrm{~N}$ $\mathrm{HNO}_{3}$ and then the Th fraction was collected by adding 3 $\mathrm{cv}$ of $6 \mathrm{~N} \mathrm{HCl}$. The Th fraction was then dried and redis-

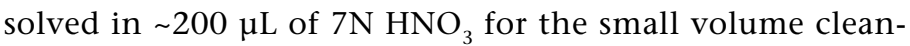
up columns. The final Th fraction was dried and taken up in $1 \mathrm{~N} \mathrm{HNO}_{3}$ for loading for TIMS.

Blanks

Chemistry blanks were processed with each batch of samples by adding Th spike, Fe carrier, $\sim 250 \mathrm{~mL}$ de-ionized water, and $1 \mathrm{~mL}$ concentrated $\mathrm{HCl}$ to a sample container and gently heating $\left(\sim 75^{\circ} \mathrm{C}\right)$ along with seawater samples for a minimum of $2 \mathrm{~d}$. Precipitation and separation chemistry followed that described above. Blanks for the 2-liter samples used to generate the baseline profile were $0.23 \pm 0.33 \mathrm{fg}\left(1.0 \pm 1.5 \times 10^{-5}\right.$ $\mathrm{dpm}){ }^{230} \mathrm{Th}$ and $8.2 \pm 5.9 \mathrm{pg}\left(2.0 \pm 1.6 \times 10^{-6} \mathrm{dpm}\right){ }^{232} \mathrm{Th}$ based on 13 measurements.

\section{Mass spectrometry}

TIMS Th analyses were performed on a Finnigan MAT 262 RPQ mass spectrometer, using a secondary electron multiplier in pulse counting mode. All filaments used for analyses were first loaded with a small amount of colloidal graphite and analyzed by TIMS at temperatures of $1750^{\circ}$ to $2050^{\circ} \mathrm{C}$ to monitor signals in the Th mass range. Filaments with count rates at masses 229 and 230 that were significantly higher than multiplier dark noise were discarded from use. All filaments had measurable 232 count rates. Those with rates higher than 500 cps were discarded. This count rate is equivalent to the beam produced by about $2 \mathrm{pg}\left(\sim 5 \times 10^{-7} \mathrm{dpm}\right)$ of ${ }^{232} \mathrm{Th}$. Correction for the 232 filament blank was included in the total chemistry blank. Samples were loaded onto the acceptable filaments using $\sim 0.1 \mathrm{~N} \mathrm{HNO}_{3}$, evaporated to dryness, covered with colloidal graphite, and then heated using a current of $\sim 2$ amps for $\sim 20 \mathrm{~s}$ to dry the graphite. Typical ion yields were $0.5 \%$.

\section{Assessment}

\section{Spiking at sea}

Sample integrity during transportation and storage was tested in two steps. The first step determined whether any bias was introduced by deferring the addition of chemical yield monitors until the samples (filtered and acidified) had been delivered to the shore-based laboratory. The second step, described below, determined whether or not there was a detectable change in the concentration of dissolved nuclides during storage of filtered and acidified (but unspiked) samples for periods of up to $3 \mathrm{y}$. All of the water used for these tests was drawn from the 1000-L of seawater, collected from $2000 \mathrm{~m}$ at the BATS site, and homogenized in the SAFe tanks (See "Collection of seawater") for the main intercalibration effort.

The first test consisted of three sets of samples, 12 in total. Set 1 included four samples that were spiked at sea. Nuclides were concentrated at sea by coprecipitation with $\mathrm{FeCl}_{3}$ carrier following the LDEO procedure described above. Iron hydroxide was concentrated aboard the ship by centrifugation and returned to LDEO in the centrifuge tubes. Set 2 included 5 samples that were spiked at sea, using the same isotope yield monitors that were used for Set 1, and then shipped to LDEO before the addition of $\mathrm{FeCl}_{3}$ carrier or any further processing. Set 3 included three samples that were not spiked until they were processed at LDEO. Set 3 was processed at the same time as Set 2. Sample sizes for Sets 2 and 3 were determined by weighing samples at LDEO. Sample sizes for Set 1 were of necessity determined by measuring their volume in a graduated cylinder at sea and, therefore, those sample sizes may have a larger but indeterminable uncertainty.

Concentrations of ${ }^{232} \mathrm{Th}$ (Fig. 1A) and ${ }^{230} \mathrm{Th}$ (Fig. 1C) are systematically lower in the samples for which the nuclides were concentrated by co-precipitation at sea (samples 1- 4 in Fig. 1 ). The reason for this offset is unknown. Concentrations of ${ }^{232} \mathrm{Th}$ and ${ }^{230} \mathrm{Th}$ in samples spiked at sea but co-precipitated at LDEO (samples 5-9) are indistinguishable from concentra- 


\section{BATS 2000 m Seawater}
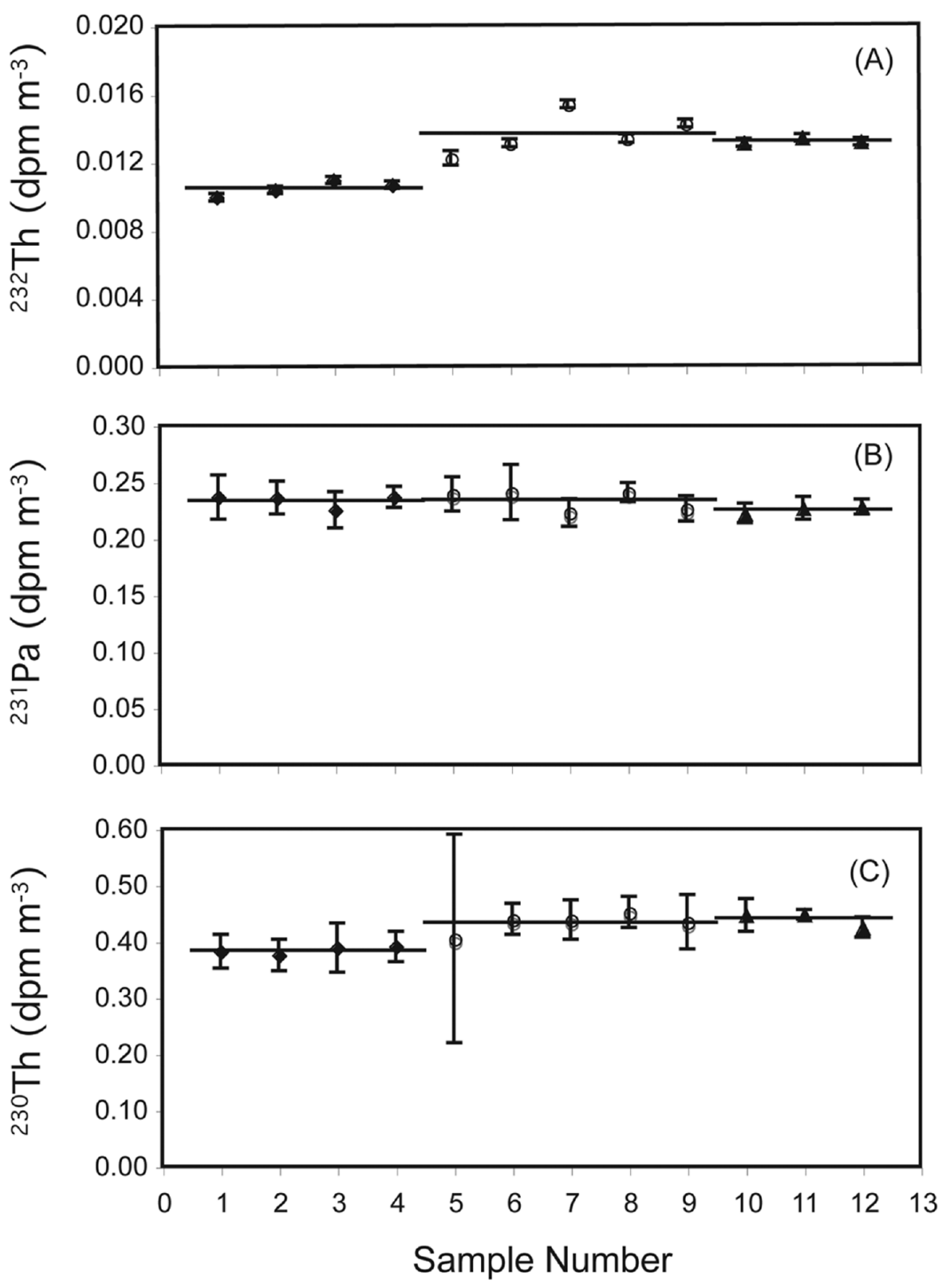

Fig. 1. Results from the test of sample storage effects during transport from the ship to the shore-based lab (LDEO). All samples were collected at a depth of $2000 \mathrm{~m}$ at the BATS site using the U.S. GEOTRACES carousel equipped with GO-Flo bottles. Water was filtered, and then acidified and homogenized in the $1000 \mathrm{~L}$ SAFe tank system (See "Collection of seawater"). Isotope yield monitors ( ${ }^{233} \mathrm{~Pa}$ and ${ }^{229} \mathrm{Th}$ ) were added to samples $1-9$ at sea. Isotope yield monitors were added to samples 10-12 when they were processed at LDEO. Iron carrier added to samples 1-4 was precipitated and concentrated at sea. Iron carrier was added to samples 5-12 when they were processed, together, at LDEO. Error bars represent the propagation of one sigma errors based on the mean and standard deviation of five sequences of isotope ratios collected by ICP-MS, the estimated error in the ${ }^{229}{ }^{2} \mathrm{Th}$ or ${ }^{233} \mathrm{~Pa}$ spike concentrations, and the blank correction of the individual isotopes for each sample batch. Horizontal lines represent mean values for each set.

tions in samples that were spiked after the cruise, in the lab at LDEO (samples 10-12). Therefore, we rule out an artifact created by delaying the spiking until the samples were processed at LDEO. An overestimation of the size of samples processed at sea (1-4) could account for lower Th concentrations in those samples, but the uniformity of ${ }^{231} \mathrm{~Pa}$ concentrations throughout all of the samples (Fig. 1B) is inconsistent with this explanation, and it is unlikely that sample volumes would be in error by as much as $10 \%$. Leaching of Th from sample containers during transport and storage is unlikely as well, 

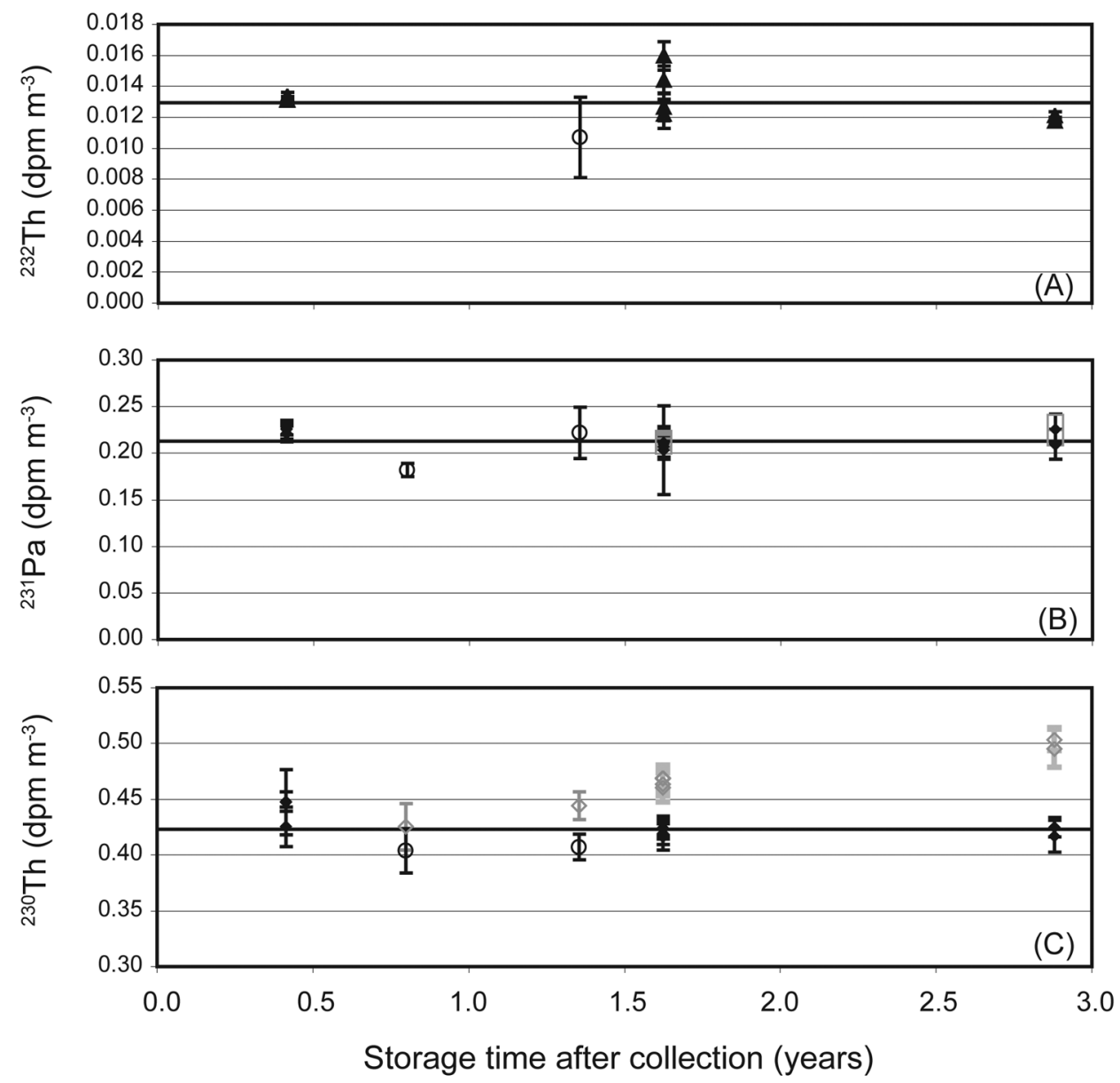

Fig. 2. Concentrations of $(A){ }^{232} \mathrm{Th},(B){ }^{231} \mathrm{~Pa}$, and $(\mathrm{C}){ }^{230} \mathrm{Th}$ determined after increasing duration of sample storage. All samples were collected at a depth of $2000 \mathrm{~m}$ at the BATS site. Solid symbols indicate samples that were collected using the U.S. GEOTRACES carousel equipped with GO-Flo bottles and homogenized in the 1000-liter SAFe tank. Open symbols indicate samples collected in Niskin bottles deployed on the ship's rosette. In (B) and (C), the black symbols have been corrected for ingrowth of ${ }^{231} \mathrm{~Pa}$ and ${ }^{230} \mathrm{Th}$, respectively, whereas the gray symbols have not been corrected. A high procedural blank during the analysis of the first Niskin sample precluded the determination of a meaningful ${ }^{232}$ Th concentration. The solid horizontal line indicates the mean concentration of each nuclide (corrected for ingrowth in B and C).

because this would require the source of leached Th to have a ${ }^{232} \mathrm{Th} /{ }^{230} \mathrm{Th}$ ratio similar to that of seawater, roughly two orders of magnitude smaller than the ratio in average crustal material, a more likely source of contamination. Furthermore, clean but unused sample containers were filled at sea with distilled water and processed as blanks. They showed no evidence for leaching of Th from the container material. Iron carrier and ammonia used to process samples at sea came from the same batches as the reagents used at LDEO, so contamination of these reagents is also unlikely to be responsible for the observed differences.

Unfortunately, variability among results for the main intercalibration exercise using samples from the 2000-m SAFe tank was so large (see "Discussion" below pertaining to Fig. 7) that they are of little help in determining which set of results in Fig. 1 is more accurate. Nevertheless, if one makes an informed subjective decision about the consensus values for ${ }^{232} \mathrm{Th}$ and ${ }^{230} \mathrm{Th}$ based on the main intercalibration samples (see text below related to Fig. 7), then results from sets 2 and 3 (samples 5-12 in Fig. 1) are more consistent with the consensus values than are the results from Set 1.

Whatever the cause of the lower Th concentrations in samples coprecipitated at sea (Set 1), results from this test demonstrate that $\mathrm{Th}$ is not lost from seawater samples acidified to $\mathrm{pH}$ $\sim 1.7$ during transport from the ship to the shore-based lab.

\section{Sample storage}

Analysis of samples collected during KN193-6 showed no systematic trend in concentration of ${ }^{230} \mathrm{Th},{ }^{232} \mathrm{Th}$, or ${ }^{231} \mathrm{~Pa}$ over a 3-y period following collection in July 2008 (Fig. 2). Thus, we conclude that samples may be stored for a period of at least 3 $\mathrm{y}$ without loss of Th or of $\mathrm{Pa}$ by precipitation or by sorption to container walls, provided that samples are filtered and acidified to $\mathrm{pH} \sim 1.7$, and provided that appropriate corrections for ingrowth are made.

Corrections for ${ }^{230} \mathrm{Th}$ ingrowth must be made if samples are stored for more than a few months (see Fig. 8 in Robinson et 
al. 2004). Concentrations of dissolved ${ }^{230} \mathrm{Th}$ in seawater are on the order of $0.01 \%$ of the level that would occur if ${ }^{230} \mathrm{Th}$ was in radioactive secular equilibrium with ${ }^{234} \mathrm{U}$ (this is an approximate value as the concentration of ${ }^{230} \mathrm{Th}$ increases with depth in the water column whereas the concentration of its parent, ${ }^{234} \mathrm{U}$, is relatively uniform throughout the ocean). Low concentrations of dissolved ${ }^{230} \mathrm{Th}$ in the ocean are a consequence of its high affinity for sorption to particle surfaces. Dissolved ${ }^{230} \mathrm{Th}$ concentrations in filtered and acidified samples increase at a rate of about $0.027 \mathrm{dpm} \mathrm{m}^{-3} \mathrm{y}^{-1}$ (or $\sim 0.57 \mathrm{fg} \mathrm{kg}^{-1} \mathrm{y}^{-1}$ ) due to ingrowth and the elimination of these removal processes (the actual rate varies from sample to sample depending on the salinity and concentration of uranium). The impact of ingrowth is evident in Fig. 2C where concentrations of ${ }^{230} \mathrm{Th}$ uncorrected for ingrowth (gray symbols) diverge from the corrected values (black symbols) by an amount that increases with storage time.

Ingrowth corrections were made assuming a constant ratio of dissolved uranium to salinity (Chen et al. 1986) and the measured salinity at each sample location (often using the CTD results rather than bottle salinity). However, there is evidence that the U/Salinity ratio may not be constant in some cases (Owens et al. 2011; Robinson et al. 2004). Errors introduced by assuming a constant U/Salinity ratio will be small for short sample storage times, but it may be necessary to measure the concentration of uranium when making ingrowth corrections for ${ }^{230} \mathrm{Th}$ if samples are stored for long periods of time.

Here we corrected concentrations of dissolved ${ }^{231} \mathrm{~Pa}$ for ingrowth as well although the correction, expressed as percent of the initial concentration, was much smaller than for ${ }^{230} \mathrm{Th}$. Ingrowth imposes a smaller factor for ${ }^{231} \mathrm{~Pa}$ because its concentrations in the deep sea are on the order of $0.2 \%$ of those that would occur in the case of radioactive secular equilibrium with ${ }^{235} \mathrm{U}$. Although still low, this is significantly greater than for ${ }^{230} \mathrm{Th}(\sim 0.01 \%)$, reflecting the lower affinity of Pa for sorption to most particulate phases (excepting biogenic opal and some metal oxide phases, e.g., Anderson et al. 1983a, 1983b). After 3 y storage, the ingrowth corrections were still at about the level of the overall analytical uncertainty in the measurements (see gray symbols in Fig. 2B).

\section{Niskin versus GO-Flo}

The storage experiment offered an opportunity to test for contamination by sampling with standard Niskin bottles. The primary samples for the storage tests were taken from the SAFe tanks, which were filled using five casts of the U.S. GEOTRACES rosette where all 24 GO-Flo bottles were tripped at a depth of 2000 m (See "Collection of seawater"). Results from two samples of seawater collected at $2000 \mathrm{~m}$ using the Niskin rosette and analyzed as part of this study are shown as open circles in Fig. 2. Neither Niskin sample shows evidence for contamination. If anything, concentrations of ${ }^{230} \mathrm{Th}$ and ${ }^{232} \mathrm{Th}$ are slightly lower in the Niskin samples, although the concentrations overlap within the reported analytical uncertainty. The lower Th concentrations in Niskin samples might indicate the loss of Th by sorp- tion to bottle walls, but it could also reflect natural variability created by the fact that the Niskin and GO-Flo casts were taken on different days and therefore do not reflect true replicate samples. A larger number of analyses would be necessary to evaluate this potential bias more rigorously.

\section{Spike equilibration}

The corollary to the question "how long can samples be stored?" is "how long must yield monitors equilibrate before samples can be processed?" Isotopes added in acidic solutions as yield monitors would be expected to have a chemical speciation very different from that of isotopes of the same element dissolved in seawater. Acidifying the seawater sample before addition of the yield monitors may partly compensate for this, but it is still unclear if the speciation is fundamentally different, or how much time is required for isotopes added as yield monitors to equilibrate with the isotopes present naturally in the sample.

Traditionally, the authors and other investigators have allowed at least a day for spikes to equilibrate with sample isotopes before extracting the nuclides from bulk seawater, and this was the case for samples coprecipitated at sea during the first intercalibration cruise (See "Spiking at sea"). To test whether this is sufficient, or even necessary, a suite of $1-\mathrm{L}$ aliquots of deep-sea water were spiked with a dual ${ }^{229} \mathrm{Th}$ and ${ }^{236} \mathrm{U}$ spike at the University of Oxford. Uranium and Th were extracted from the seawater by coprecipitation with iron hydroxide at various times after the spikes were added, purified, and analyzed by MC-ICP-MS. Results of these analyses (Fig. 3) indicate that the traditional one-day spike equilibration should be sufficient. If anything, the isotopes of "insoluble" thorium (Fig. 3A, B) seem to reach their final values very rapidly, whereas uranium (Fig. $3 \mathrm{C}$ ) may take up to a day to equilibrate, as indicated by the asymptotic approach toward final values of ${ }^{238} \mathrm{U}$ concentration. Rapid equilibration with a Th isotope yield monitor was also observed in a study of ${ }^{234} \mathrm{Th}$ (Wei and Hung 1993). Together, these results indicate that the common practice of allowing $1 \mathrm{~d}$ between spiking and coprecipitation is more than adequate for Th, but a full day may be necessary for spike equilibration when measuring $U$ concentrations in seawater.

\section{Test of different filters}

Historically, various types of filter material have been used by different labs when determining concentrations of dissolved trace elements and radionuclides, but no systematic test had been made to compare results for Th and Pa using different filter materials. Participants in this intercalibration felt that such a test was necessary to establish whether all labs should use the same type of filters, or if each lab could use its preferred filter type without introducing a significant bias.

Filter tests were conducted by tripping all 24 bottles on the Niskin rosette at a single depth. The entire contents of each Niskin bottle were filtered through a single filter, except in a few cases where Nuclepore membranes had to be changed in mid filtration due to clogging. Tests were conducted at two 


\section{Spike Equilibration}

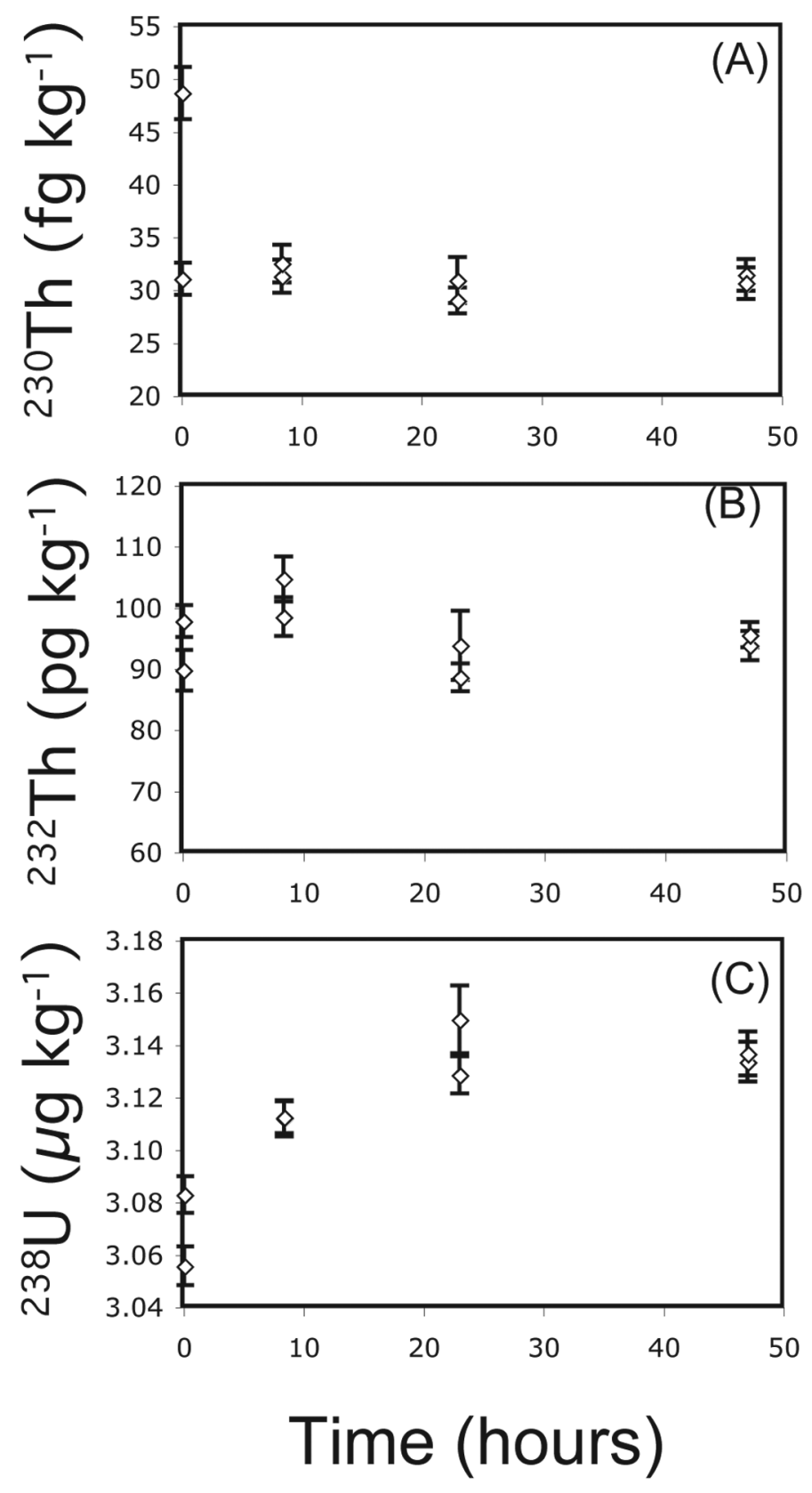

Fig. 3. Concentrations of (A) ${ }^{230} \mathrm{Th},(B){ }^{232} \mathrm{Th}$, and (C) ${ }^{238} \mathrm{U}$ determined at the University of Oxford as a function of time after a suite of $1-L$ aliquots of a deep-sea water sample were spiked with isotope yield monitors. The time axis quantifies the interval between spike addition and coprecipitation.

locations during KN193-6: (1) at a depth of $2000 \mathrm{~m}$ at the BATS station, corresponding to the location of water collected for intercalibration, and roughly corresponding to the mid depth minimum in particle concentration, and (2) at a depth of $635 \mathrm{~m}$ at a site over the continental slope $\left(37^{\circ} 2.0^{\prime} \mathrm{N}\right.$, $74^{\circ} 23.87^{\prime} \mathrm{W}$ ) where the concentration of particles was expected to be greater than at the BATS site. Particle concentrations at these two sites were expected to span the approximate range of concentrations in subsurface waters on openocean sections. Although dry weights of particle mass were not determined, transmissometer profiles collected during full depth casts at each station confirmed that the 2000-m depth at the BATS station was close to the mid depth particle minimum, and that concentrations of particles at the slope station were much greater than at the BATS station (data not shown). A smaller selection of filter types was compared at the slope station than at the BATS site.

Filtration through all types of filters reduced the concentrations of ${ }^{230} \mathrm{Th}$ and ${ }^{232} \mathrm{Th}$ substantially compared to unfiltered samples at both sites (Figs. 4 and 5). Concentrations of ${ }^{231} \mathrm{~Pa}$ were not significantly lower in filtered samples, consistent with the lower affinity of Pa for particles compared to Th. A larger fraction of the total (unfiltered) Th concentration was associated with filterable particles (i.e., unfiltered minus average filtered Th concentration) at the slope station than at the BATS site $\left(18 \%\right.$ of ${ }^{230} \mathrm{Th}$ at BATS; $37 \%$ of ${ }^{230} \mathrm{Th}$ at the slope station), as expected given the greater abundance of particles at the slope site. A larger fraction of the total concentration of ${ }^{232} \mathrm{Th}$ was associated with filterable particles than for ${ }^{230} \mathrm{Th}$ ( $31 \%$ of ${ }^{232} \mathrm{Th}$ at the BATS site versus $18 \%$ for ${ }^{230} \mathrm{Th}$ ), which is also expected given that there is no in situ production of dissolved ${ }^{232} \mathrm{Th}$ by radioactive decay of a parent isotope.

Among the standard materials that were tested as individual filters of 47 or $90-\mathrm{mm}$ diameter (Nuclepore, QMA, Supor) there was no detectable difference in the concentrations of any of the three nuclides (Figs. 4 and 5). Furthermore, there was no systematic difference between pore diameters ( 0.4 $\mu \mathrm{m}$ versus $1.0 \mu \mathrm{m}$ for Nuclepore; $0.45 \mu \mathrm{m}$ versus $0.8 \mu \mathrm{m}$ for Supor). This latter comparison suggests that most of the particulate Th that is retained by $0.4 \mu \mathrm{m}$ pore diameter filters, as estimated by comparing Th concentrations in filtered and in unfiltered samples, resides on particles greater than $1.0 \mu \mathrm{m}$ in diameter. By contrast, concentrations of Th (both ${ }^{232} \mathrm{Th}$ and ${ }^{230} \mathrm{Th}$ ) were systematically (albeit slightly) lower in samples filtered through Osmonics cartridges with $0.22 \mu \mathrm{m}$ pore diameter membranes. Whether the lower Th concentrations in samples filtered through the Osmonics cartridges reflect the presence of a significant fraction of Th on particles between 0.2 and $0.4 \mu \mathrm{m}$ in diameter, or reflect sorption of dissolved Th to the filter cartridges, cannot be assessed with the existing information. The Osmonics cartridge used here was of the same type used to filter $1000 \mathrm{~L}$ seawater when filling the SAFe tanks (1.6 square meters effective surface area; see above), and thus it was much larger than the filters and filter holders used for the other filtration tests (either 47-mm or $90-\mathrm{mm}$ diameter). Therefore, dissolved Th may have been lost by sorption to the Osmonics cartridge or to its filter membrane. Also, the dead volume inside the Osmonics cartridge was more than $1 \mathrm{~L}$, and it is possible that the cartridge was flushed insufficiently before sample collection com- 

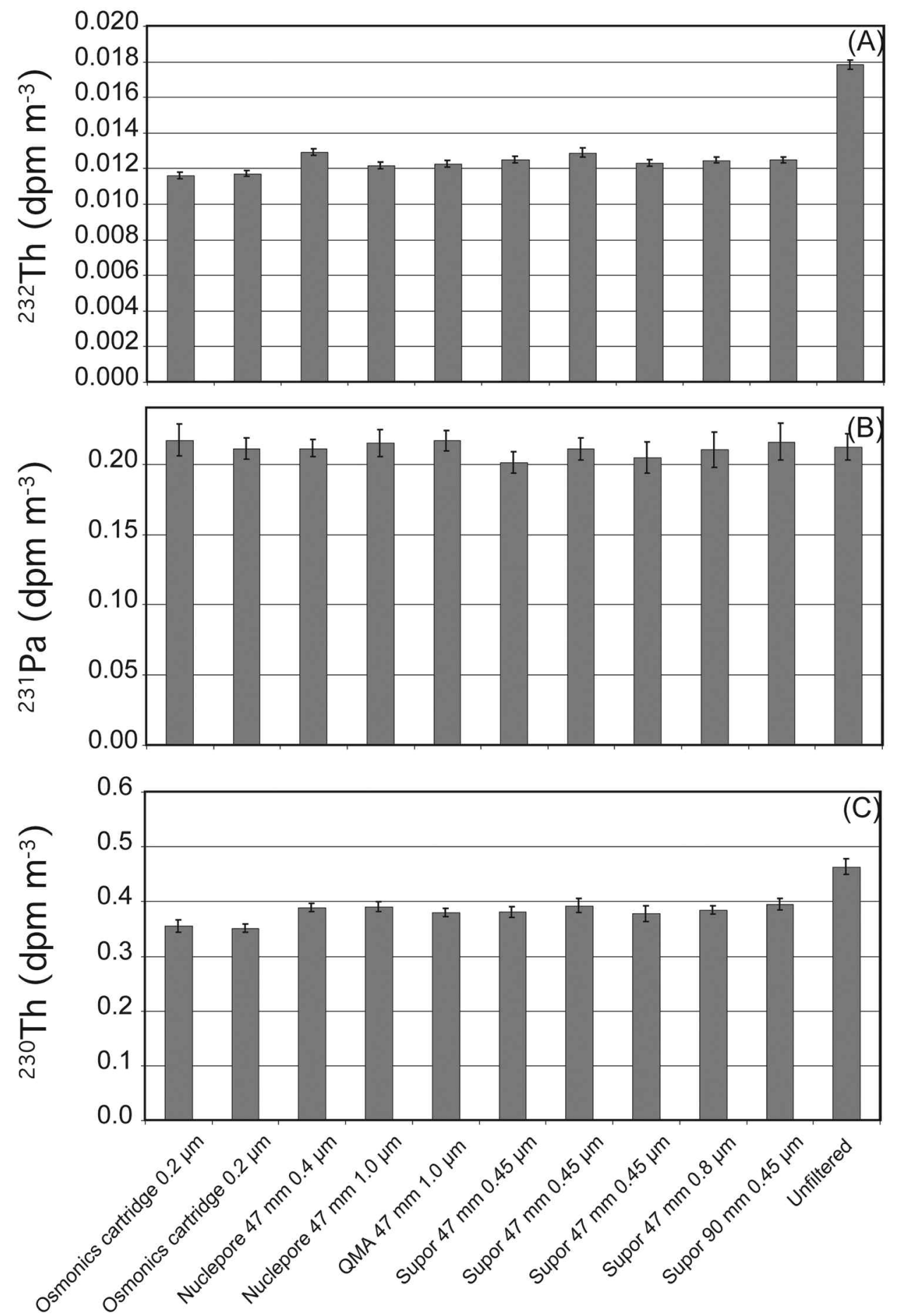

Fig. 4. Concentrations of $(A){ }^{232} \mathrm{Th},(B){ }^{231} \mathrm{~Pa}$, and $(\mathrm{C}){ }^{230} \mathrm{Th}$ determined by filtering seawater collected in individual Niskin bottles that were all closed at a depth of $2000 \mathrm{~m}$ on the same cast at the BATS site. The type of filter used in each case is indicated across the bottom, along with filter diameter (mm) and nominal pore size $(\mu \mathrm{m})$.

menced. Although cartridges as large as this might not be used to filter routinely from Niskin bottles, intermediate-sized filtration cartridges may be used on certain cruises to filter all samples consecutively in a clean van before distributing the unfiltered samples. This is an excellent way to filter large vol- umes of samples under the cleanest possible conditions, similar to the method used for trace metals. Consequently, we recommend that future studies should be carried out to determine the fraction of $\mathrm{Th}$ in the deep sea that resides on particles smaller than $0.4 \mu \mathrm{m}$ in diameter, and to determine if 

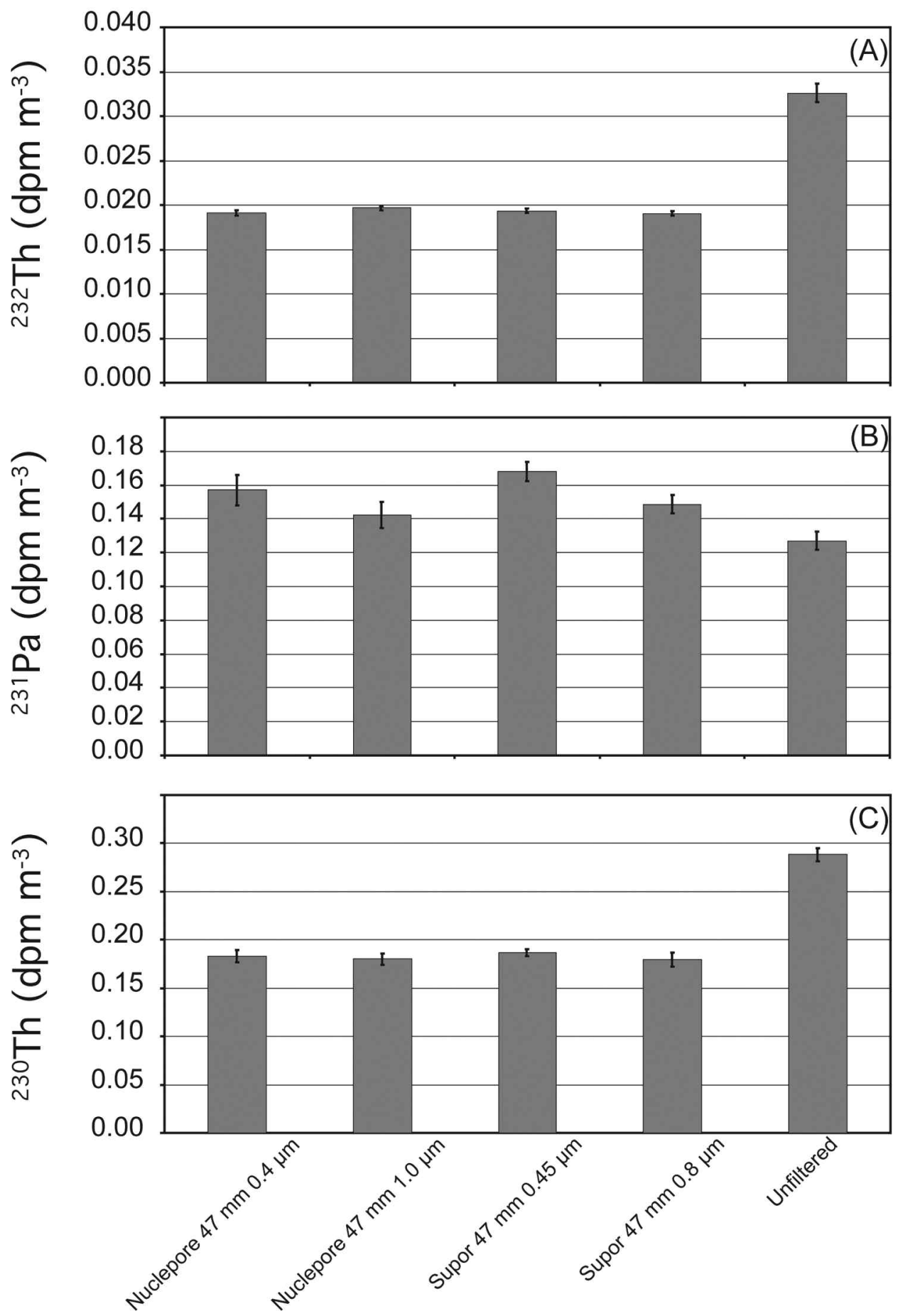

Fig. 5. Concentrations of $(A){ }^{232} \mathrm{Th},(B){ }^{231} \mathrm{~Pa}$, and (C) ${ }^{230} \mathrm{Th}$ determined by filtering seawater collected in replicate Niskin bottles from a depth of $635 \mathrm{~m}$ at a site on the continental slope off the east coast of the U.S. $\left(37^{\circ} 2.0^{\prime} \mathrm{N}, 74^{\circ} 23.87^{\prime} \mathrm{W}\right)$. The type of filter used in each case is indicated across the bottom along with filter diameter $(\mathrm{mm})$ and nominal pore size $(\mu \mathrm{m})$.

sampling biases are introduced when using large filtration cartridges.

Concentrations of dissolved nuclides obtained by filtration through quartz (QMA) filters were not significantly different from those obtained with the individual (47 or $90 \mathrm{~mm}$ ) mem- brane filters for any of the three nuclides (Fig. 4). This is somewhat surprising because $\mathrm{Pa}$ is known to have a high affinity for sorption to glass and to amorphous silica (e.g., many labs use silica gel to adsorb ${ }^{233} \mathrm{~Pa}$ when milking it from ${ }^{237} \mathrm{~Np}$ ). Further testing would be necessary before we would confidently con- 


\section{Siliceous Ooze Sediments}
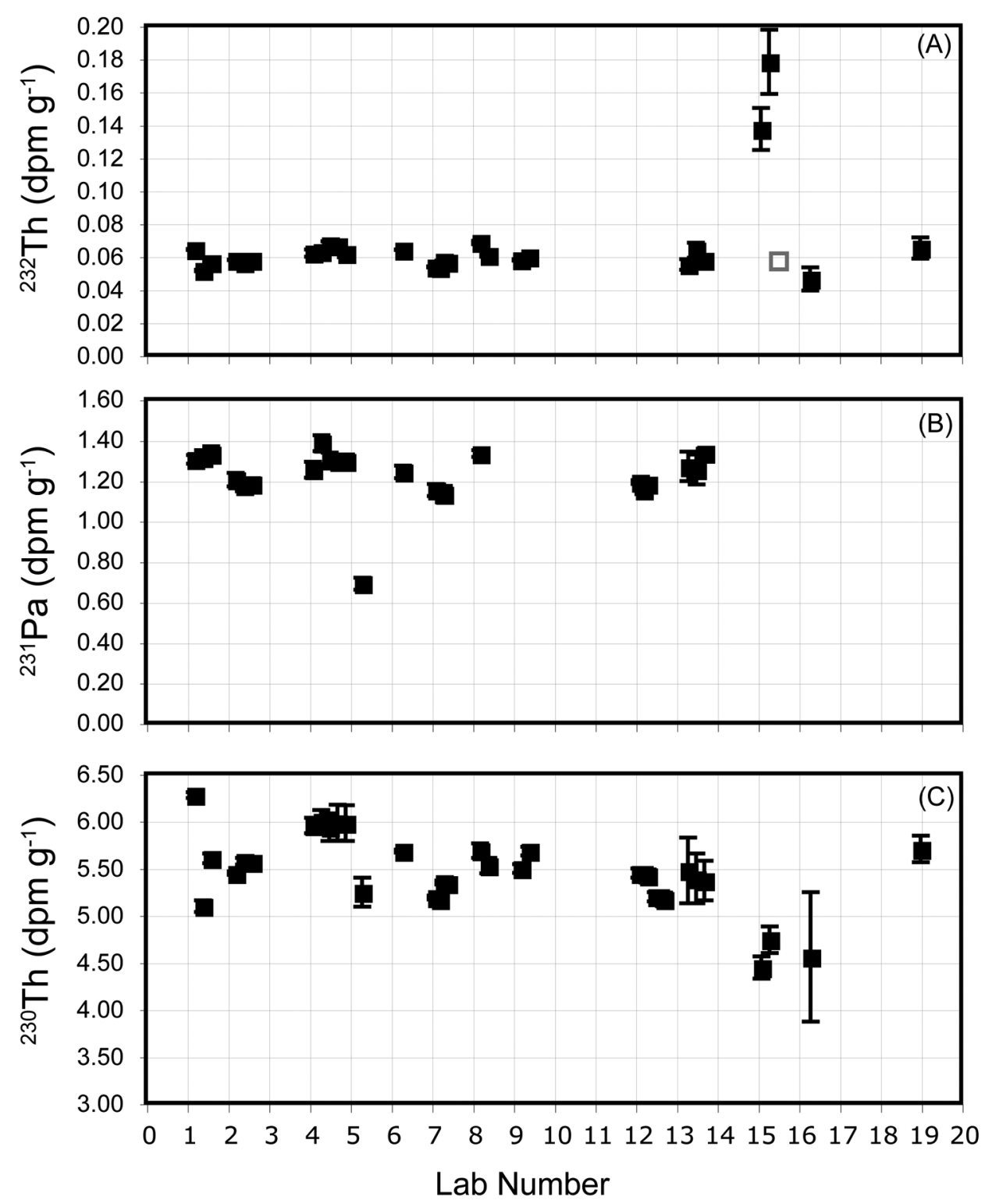

Fig. 6. Concentrations of $(A){ }^{232} \mathrm{Th},(B){ }^{231} \mathrm{~Pa}$, and (C) ${ }^{230} \mathrm{Th}$ determined by analyzing aliquots of homogenized siliceous ooze from the Southern Ocean. Participating laboratories are identified anonymously by Lab Number across the bottom. The hollow gray symbol for Lab 15 in (A) represents the result from reanalysis of the sample by ICP-MS subsequent to the original analysis by $\alpha$ spectrometry.

clude that QMA filters may be used to determine concentrations of dissolved Th and Pa on GEOTRACES cruises.

\section{Intercalibration-Sediment analyses}

The most comprehensive assessment of intercalibration among labs that measure $\mathrm{Th}$ and $\mathrm{Pa}$ was provided by the analysis of siliceous ooze sediments. Concentrations of ${ }^{232} \mathrm{Th}$ agreed nearly as well between labs as between replicated analyses from a single lab with the exception of two high values reported initially by Lab 15 using $\alpha$ spectrometry (Fig. 6A). When Lab 15 reanalyzed the sample by ICP-MS (hollow gray symbol in Fig. 6A) the ${ }^{232} \mathrm{Th}$ concentration fell into line with the values from other labs. The source of the high ${ }^{232} \mathrm{Th}$ values remains undetermined, but it may involve an insufficient tail correction in the $\alpha$ spectrum. Excluding these high values, the mean and standard deviation of lab mean values (i.e., averaging for each lab where replicate results were reported) is 0.059 $\pm 0.005 \mathrm{dpm} / \mathrm{g}(0.242 \pm 0.021 \mu \mathrm{g} / \mathrm{g} ; 9 \% 1 \mathrm{sigma})$.

Reported concentrations of ${ }^{230} \mathrm{Th}$ vary by as much as $20 \%$ (full range of values) among labs (Fig. 6C), more than would be expected given the agreement for ${ }^{232} \mathrm{Th}$. That is, the variability does not seem to be due to differences among labs in the calibration of the ${ }^{229} \mathrm{Th}$ yield monitors, with the possible 

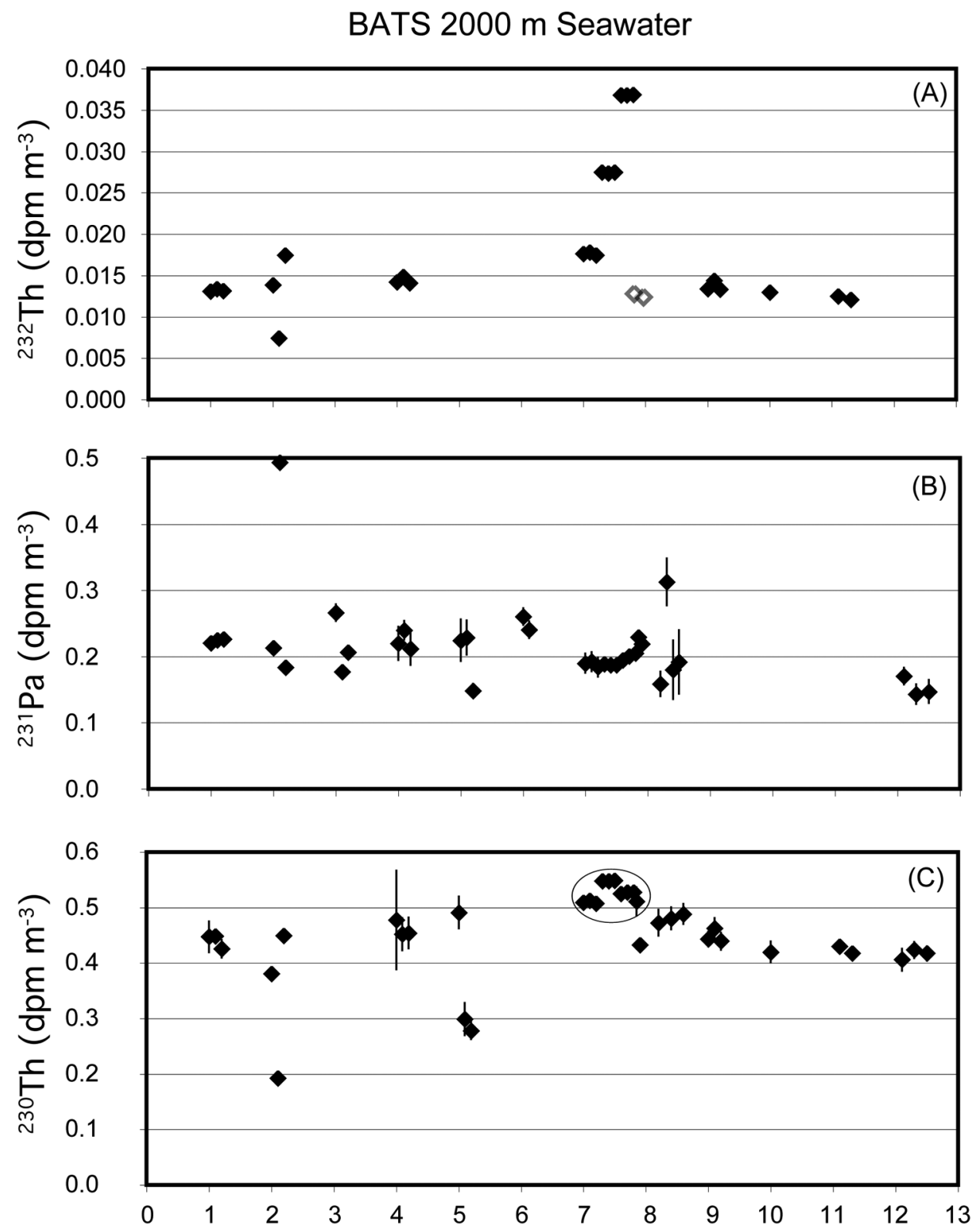

\section{Lab Number}

Fig. 7. Concentrations of (A) ${ }^{232} \mathrm{Th},(B){ }^{231} \mathrm{~Pa}$, and (C) ${ }^{230} \mathrm{Th}$ determined by analyzing aliquots of homogenized seawater collected from a depth of 2000 $\mathrm{m}$ at the BATS site during the first intercalibration cruise. Samples were drawn from the 1000-L SAFe tank system that had been filled using repeated casts of the U.S. GEOTRACES carousel, equipped with GO-Flo bottles. Water was filtered as it was transferred from the GO-Flo bottles to the tanks and acidified to a $\mathrm{pH} \sim 1.7$. Hollow gray points for Lab 7 in (A) are from 1-L samples that were archived separately during the cruise and analyzed by Lab 7 subsequent to the discovery of Th contamination in their larger samples (see "Intercalibration-Seawater analyses"). Circled results in (C) indicate samples that were flagged by Lab 7 as being contaminated, corresponding to their high ${ }^{232}$ Th values (but not circled in A). Lab numbers are unchanged from Fig. 6.

exception of Lab 16 for which the reported ${ }^{232} \mathrm{Th}$ and ${ }^{230} \mathrm{Th}$ values were each about $20 \%$ less than the respective means of the other labs. Including results from all labs, the mean and standard deviation of lab mean values is $5.38 \pm 0.42 \mathrm{dpm} / \mathrm{g}(7.7 \%$ 1 sigma). Excluding the low mean values from Labs 15 and 16, the mean and standard deviation of the lab mean values for the remaining 11 labs becomes $5.53 \pm 0.22 \mathrm{dpm} / \mathrm{g}(4 \% 1$ sigma). Agreement for ${ }^{231} \mathrm{~Pa}$ (Fig. 6B) is similar to that for ${ }^{230} \mathrm{Th}$ with the exception of one lab (5) that reported a ${ }^{231} \mathrm{~Pa}$ concentration that was $55 \%$ of the mean value from other labs. Excluding this one low value, the mean and standard deviation of lab mean values is $1.25 \pm 0.07 \mathrm{dpm} / \mathrm{g}$ (6\% 1 sigma). 
Discussion of these results among group participants raised questions about the homogeneity of the siliceous ooze, given that variability among results from a single lab for ${ }^{230} \mathrm{Th}$ (e.g., Lab 1) is similar to the variability between all labs (Fig. 6C). Intra-lab variability is not the result of spike calibration or other systematic errors. If a large portion of the intra-lab variability evident in Fig. 6C is due to sample heterogeneity, then this is a concern for anyone measuring these nuclides in sediments. The siliceous ooze was homogenized more thoroughly than is common during the analysis of marine sediments in anticipation that it would be used for intercalibration. It would be a concern if variability among aliquots of as much as $20 \%$ survived this level of homogenization. In response to this concern about sample heterogeneity, the LDEO group prepared a mixed isotope solution containing ${ }^{232} \mathrm{Th},{ }^{230} \mathrm{Th}$, and ${ }^{231} \mathrm{~Pa}$ that could be analyzed as a working standard. Results from that solution are described in "Intercalibration-working standards" below.

\section{Intercalibration-Seawater analyses}

Initial intercalibration results from seawater collected during the first cruise (KN193-6, BATS site) revealed unexpected levels of contamination by sources of ${ }^{232} \mathrm{Th}$. Further investigation indicated that contamination was sometimes derived from sample containers and sometimes from laboratory procedures (see also accompanying papers by Andersson and Schöberg 2012; Auro et al. 2012). As noted above, there was no indication of contamination from the Niskin sampling system used at the BATS site.

Triplicate analyses by Lab 7 of each of three samples from $2000 \mathrm{~m}$ at the BATS site indicated that Th contamination was present in sample containers (Fig. 7A). Internal consistency within each set of triplicate samples, together with the large differences in ${ }^{232} \mathrm{Th}$ concentration between individual samples, is consistent with contamination of the sample containers rather than the random introduction of contamination during processing of the samples. After identifying this problem, Lab 7 analyzed samples that had been collected in a different set of containers (smaller containers also provided by Lab 7) and obtained ${ }^{232} \mathrm{Th}$ concentrations (hollow gray symbols in Fig. 7A) close to those from other labs. By plotting ${ }^{232} \mathrm{Th} /{ }^{230} \mathrm{Th}$ ratios versus ${ }^{232} \mathrm{Th}$ concentrations Lab 7 was able to establish that the source of contamination had a crustal ${ }^{232} \mathrm{Th} /{ }^{230} \mathrm{Th}$ ratio (not shown). Lab 8 also encountered ${ }^{232} \mathrm{Th}$ contamination. Their blanks were so large that they precluded reporting ${ }^{232} \mathrm{Th}$ concentrations during the first set of intercalibration tests (BATS seawater).

Contamination introduced a smaller but still significant bias in ${ }^{230} \mathrm{Th}$ concentrations, as is evident by circled values from Lab 7 in Fig. 7C, which are greater than ${ }^{230}$ Th concentrations reported by other labs. These results were flagged as contaminated by Lab 7 at the time that they were first reported.

An important lesson from this experience is that sample containers may be a significant source of contamination. Blanks must be determined using sample containers that are cleaned and handled by the same procedure as the samples. A weakness of this intercalibration exercise is that empty sample containers from each lab were not processed at sea as blanks. We recommend that blanks be processed regularly at sea. On U.S. GEOTRACES section cruises, for example, a seawater blank is processed at each station by adding 2 to 3 L Milli-Q water to a sample container and treating it as a sample.

Discovery of the unexpected contamination problems described above, as well as others that were later identified, stimulated an extensive discussion and collaboration among participants to locate sources of contamination and to eliminate them. Investigators must be cautious about checking blanks, especially when changes are made in analytical methods. For example, the LDEO authors of this manuscript discovered that a new source of high purity iron carrier was heavily contaminated with Th. Repeated extraction of iron from 7N HCl into isopropyl ether eventually reduced the contamination. Similarly, the WHOI author of this manuscript discovered that some batches of ion-exchange resin contain large amounts of Th, and that some labware adsorbs Th irreversibly, leading to low chemical yields. Those experiences are described in greater detail in a companion manuscript (Auro et al. 2012). The lesson here is that the clean-up procedure that may be acceptable for one batch of Fe carrier or resin may be inadequate for another.

As challenging as the contamination issue has proven to be, it can be (and has been) addressed through systematic testing of individual steps in the collection and analysis of seawater samples. A more perplexing problem that was discovered during the seawater intercalibration involves poor reproducibility among results from a single lab. Sometimes this is manifest as low concentrations that cannot be explained by contamination (e.g., see ${ }^{230} \mathrm{Th}$ results from Labs 2 and 5 in Fig. 7C), and sometimes as high concentrations for ${ }^{231} \mathrm{~Pa}$ in samples where no corresponding contamination of Th was evident (e.g., see ${ }^{231} \mathrm{~Pa}$ results for Labs 2 and 8 in Fig. 7B). The source(s) of this variability is (are) still under investigation.

Analysis of surface waters (from a depth of between 5 and $15 \mathrm{~m}$ ) is more challenging because of the much lower concentrations of dissolved ${ }^{230} \mathrm{Th}$ and ${ }^{231} \mathrm{~Pa}$ (compare Fig. 7 panels $\mathrm{B}$ and $\mathrm{C}$ with Fig. 8 panels $\mathrm{B}$ and $\mathrm{C}$ ). Despite the lower levels, surface ${ }^{230} \mathrm{Th}$ concentrations were within reported uncertainty of $0.08 \mathrm{dpm} \mathrm{m}^{-3}$ (Fig. 8C), except for the circled values from Lab 7, indicating results that were flagged by the investigators as likely suffering from contamination, as well as results from Lab 8, which also reported high ${ }^{232}$ Th blanks (see above). Similarly, surface concentrations of ${ }^{231} \mathrm{~Pa}$ are generally within reported error of $0.05 \mathrm{dpm} \mathrm{m}^{-3}$, except for Lab 2 and Lab 12 . Three labs reported surface concentrations of ${ }^{232} \mathrm{Th}$ within reported uncertainty of $0.02 \mathrm{dpm} \mathrm{m}^{-3}$, including the results by Lab 7 from samples collected in smaller sample containers (Fig. 8A). Concentrations of ${ }^{232}$ Th from Labs 2 and 4 are significantly higher, as are those flagged by Lab 7 as likely suffering from contamination (circled in Fig. 8A). Although results from only three labs are insufficient to conclude that 0.02 

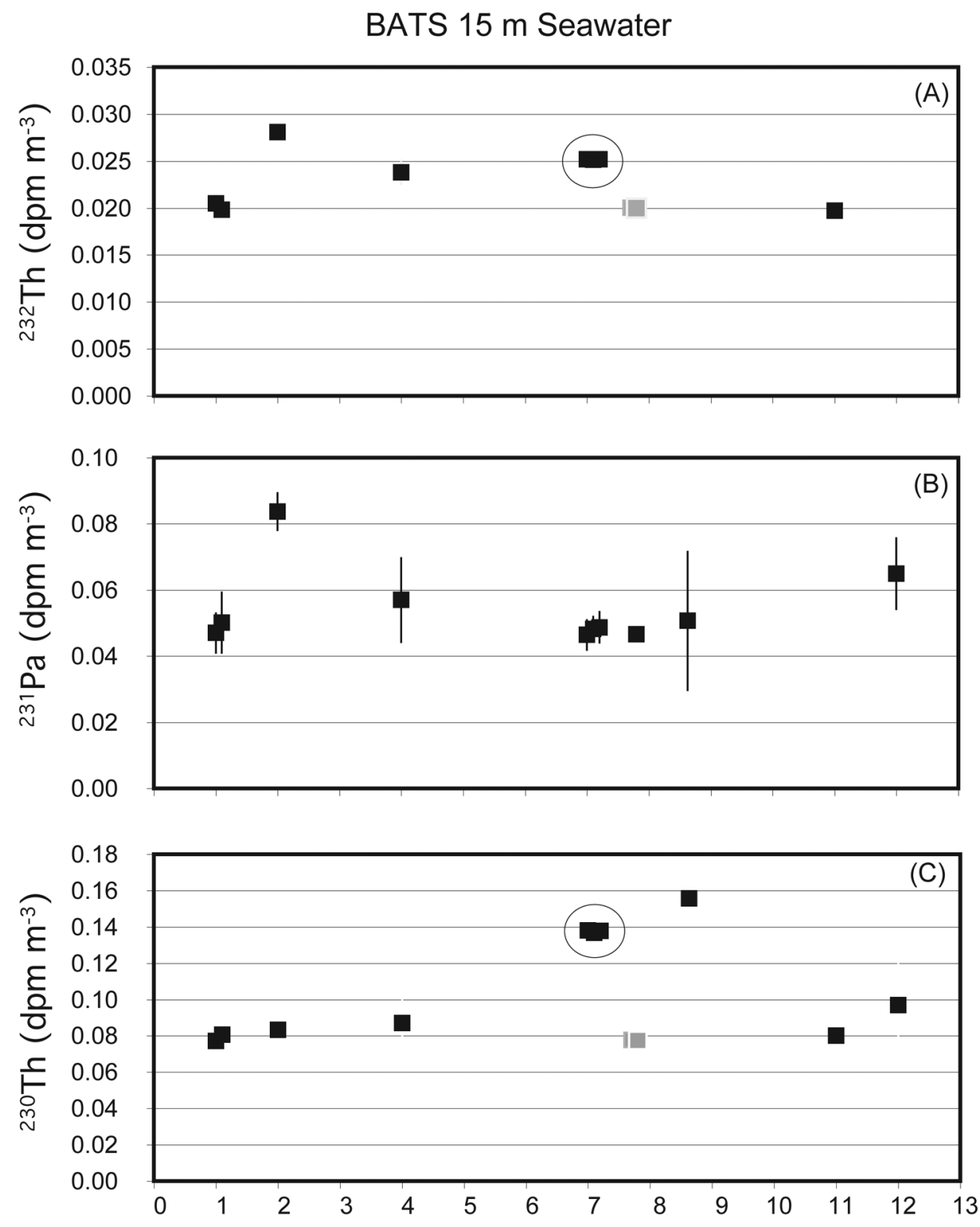

\section{Lab Number}

Fig. 8. Concentrations of $(A){ }^{232} \mathrm{Th},(B){ }^{231} \mathrm{~Pa}$, and $(\mathrm{C}){ }^{230} \mathrm{Th}$ determined by analyzing aliquots of homogenized seawater collected from a depth of $\sim 15$ $\mathrm{m}$ at the BATS site. Samples were drawn from the 1000-L SAFe tank system that had been filled using water pumped from a towed trace metal-clean fish (See "Collection of seawater"). Water was filtered as it was transferred from the fish to the tanks (See "Collection of seawater") and acidified to a pH 1.7. Circled results in (A) and (C) indicate values that were flagged by Lab 7 at the time of data submission as being contaminated. Gray points in (A) and (C) are from 1-L samples that were archived separately during the cruise and analyzed by Lab 7 subsequent to the discovery of Th contamination in their larger samples (see "Intercalibration-Seawater analyses"). Lab numbers are unchanged from Fig. 6.

$\mathrm{dpm} \mathrm{m} \mathrm{m}^{-3}$ is the correct value for the concentration of ${ }^{232} \mathrm{Th}$ in the near-surface BATS sample, the higher values from other labs would be consistent with the widespread contamination by extraneous sources of Th described above.

Evaluation and testing of Th blanks was still underway at the time of the second intercalibration cruise (KN195-8). Although the extensive efforts by participating labs in response to the results from the first cruise had improved their blank levels substantially, evidence for ${ }^{232} \mathrm{Th}$ contamination can still be found in the results from the second cruise. Lab 9 flagged high ${ }^{232} \mathrm{Th}$ values in duplicate analyses of one sample of water from $3000 \mathrm{~m}$ at the SAFe station (circled values in Fig. 9A) as having suffered from contamination. Reproducible results were obtained from duplicate analyses of this sample, indicating 
SAFe 3000 m Seawater
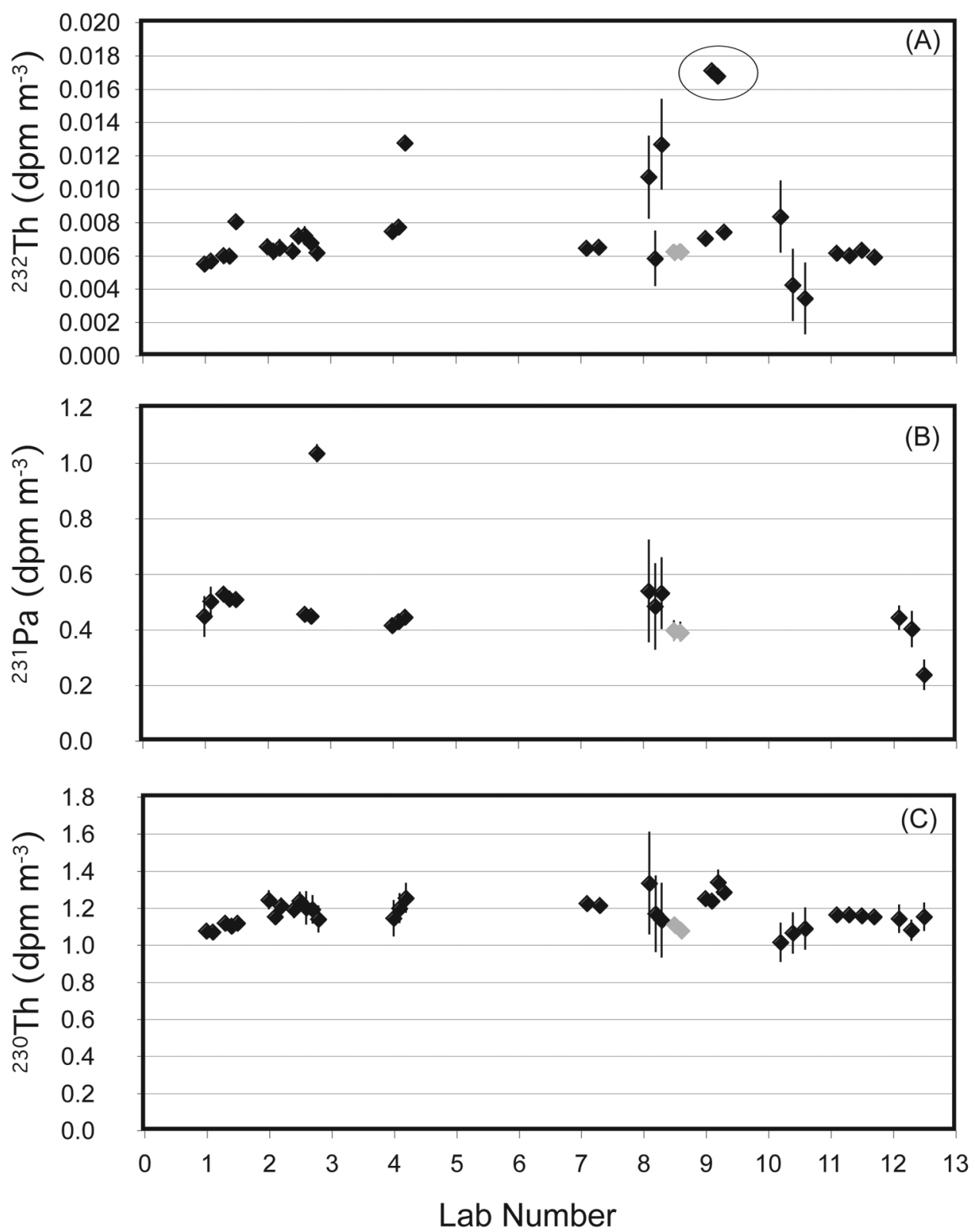

Fig. 9. Concentrations of $(A){ }^{232} \mathrm{Th},(B){ }^{231} \mathrm{~Pa}$, and $(C){ }^{230} \mathrm{Th}$ determined by analyzing aliquots of homogenized seawater collected from a depth of 3000 $\mathrm{m}$ at the SAFe site. Samples were drawn from the 1000-L SAFe tank system that had been filled using repeated casts of the U.S. GEOTRACES carousel, equipped with GO-Flo bottles. Water was filtered as it was transferred from the GO-Flo bottles to the tanks and acidified to a $\mathrm{pH} \sim 1.7$. Circled results in (A) indicate values that were flagged by Lab 9 at the time of data submission as being contaminated. Gray symbols represent results from a second set of samples that was distributed to Lab 8 after they reported that blanks had been improved substantially. Lab numbers are unchanged from Fig. 6 .

that the contamination likely occurred in the sample container rather than in the lab procedure. This is reminiscent of container contamination experienced by Lab 7 during the analysis of samples from KN193-6. The source of the container contamination is unknown. Containers were cleaned by each lab before each cruise and transported to the ship enclosed in double plastic bags. The SAFe tanks (see above), located inside a hangar aboard the ship, were enclosed within a temporary structure (tent) constructed of plastic sheeting to minimize contamination by aerosols. Sample containers were removed from their bags inside the tent, and then rinsed and filled inside the tent before rebagging for return to their final destinations. These procedures follow those adopted for collecting large numbers of replicate seawater samples for trace metal 


\section{SAFe 15 m Seawater}
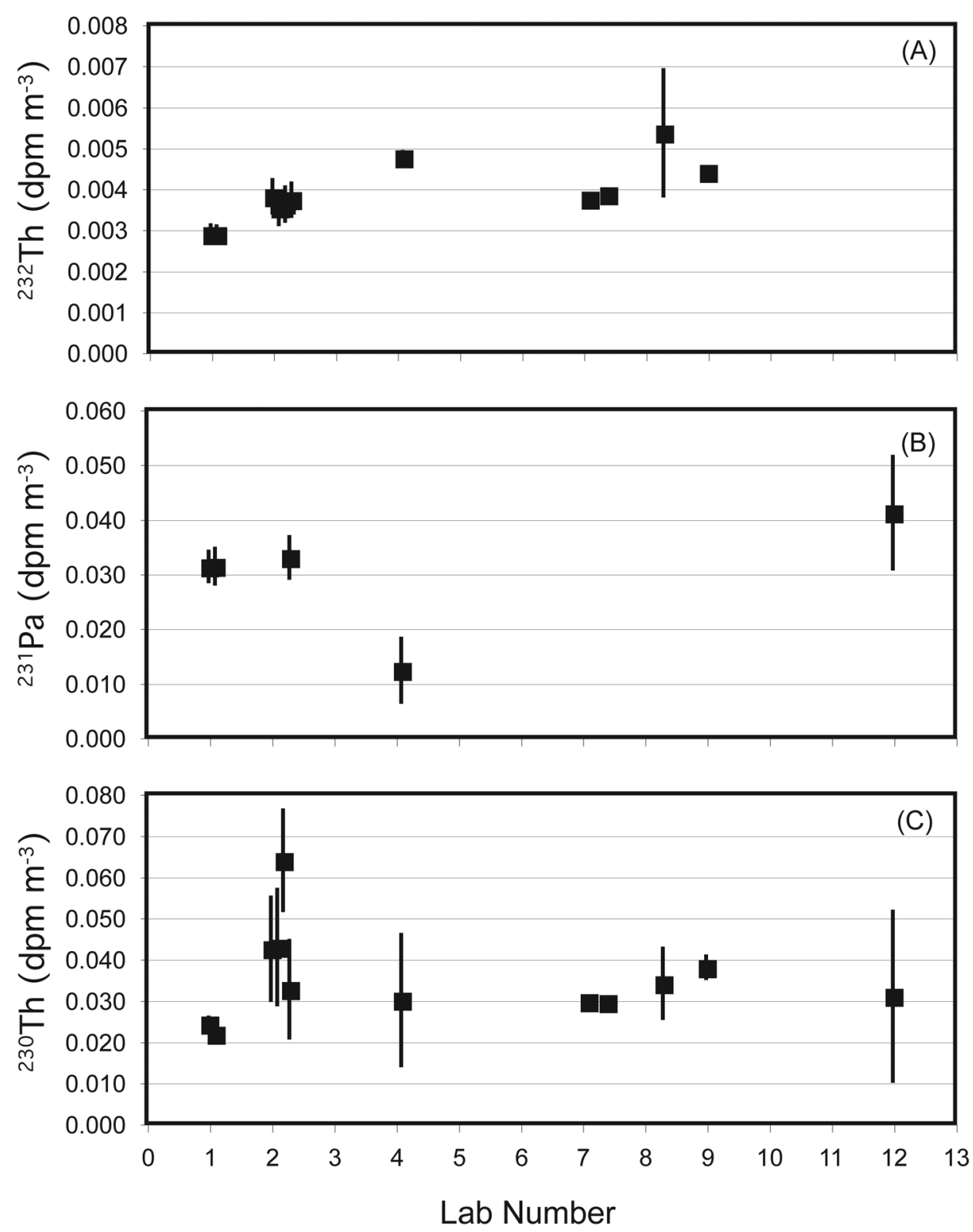

Fig. 10. Concentrations of $(A){ }^{232} \mathrm{Th},(B){ }^{231} \mathrm{~Pa}$, and $(\mathrm{C}){ }^{230} \mathrm{Th}$ determined by analyzing aliquots of homogenized seawater collected from a depth of $\sim 15$ $\mathrm{m}$ at the SAFe site. Samples were drawn from the 1000-L SAFe tank system that had been filled using water pumped from a towed trace metal-clean fish (See "Collection of seawater"). Water was filtered as it was transferred from the fish to the tanks and acidified to a pH $\sim 1.7$. Lab numbers are unchanged from Fig. 6.

analysis, which have been found to be free of trace metal contamination. Consequently, the source of Th contamination in containers remains undetermined. Lab 8 reported that they were still undertaking tests to reduce their Th blanks at the time these samples were analyzed, and the variability among the initial results for Lab 8 (Fig. 9A, black points) indicate that their efforts were initially only partially successful. Following further work to improve their blank, Lab 8 was sent two additional samples that had been archived at LDEO. Results from those samples (gray points in Fig. 9) are in good agreement with the results from other labs, consistent with the much lower blanks reported by Lab 8 at the time those samples were analyzed. Note that the results for ${ }^{231} \mathrm{~Pa}$ reported by Lab 8 also improved after their ${ }^{232} \mathrm{Th}$ blanks were lowered (gray points in Fig. 9B), reflecting in part the reduced uncertainty in the tail correction for ${ }^{232} \mathrm{Th}$ that remained in the $\mathrm{Pa}$ fraction. Lab 10 indicated that their ${ }^{232} \mathrm{Th}$ analyses required large blank corrections, adding to the variability among their results (Fig. 9A). 
Lab 10 has subsequently reported that their ongoing methods development, stimulated by their participation in the GEOTRACES intercalibration, has reduced their ${ }^{232} \mathrm{Th}$ blank level by about a factor of four since their SAFe samples were analyzed.

Determining concentrations of ${ }^{232} \mathrm{Th}$ in surface waters at the SAFe station was more challenging than at BATS because ${ }^{232} \mathrm{Th}$ concentrations in surface waters of the central Pacific are much lower than in the North Atlantic, reflecting the much lower fluxes of mineral aerosols (dust, the principal source of ${ }^{232} \mathrm{Th}$ in the open ocean) to the Pacific. The average concentration of ${ }^{232} \mathrm{Th}$ in the surface sample from the SAFe station $(0.0041 \pm$ $0.0009 \mathrm{dpm} \mathrm{m}^{-3}$; mean and standard deviation of lab mean values; Fig. 10A) is only about $20 \%$ of the average surface concentration at the BATS site $\left(0.022 \pm 0.004 \mathrm{dpm} \mathrm{m}^{-3}\right.$; mean and standard deviation of lab mean values excluding values flagged by Lab 7, Fig. 8A). Despite the low concentration of dissolved ${ }^{232} \mathrm{Th}$, high levels of contamination are not evident in any of the reported results. Labs 1 and 4 reported ${ }^{232}$ Th concentrations below and above the group mean, respectively, by more than their reported uncertainties. Blank corrections were reported to be substantial for each of these labs, so the departures from the mean ${ }^{232} \mathrm{Th}$ concentration may reflect an inaccurate evaluation of the blank (i.e., an overestimation and an underestimation of the blank correction by Labs 1 and 4, respectively, with a corresponding underestimation of the propagated uncertainty).

Results from the analysis of deep $(3000 \mathrm{~m})$ water from the SAFe station indicate an improvement for ${ }^{230} \mathrm{Th}$ and for ${ }^{231} \mathrm{~Pa}$ relative to results from the first cruise (compare Fig. 7 panels $\mathrm{B}$ and $\mathrm{C}$ with Fig. 9 panels $\mathrm{B}$ and $\mathrm{C}$ ). The mean and standard deviation of lab mean values for ${ }^{230} \mathrm{Th}$ at $3000 \mathrm{~m}$ (Fig. 9C) is $1.16 \pm 0.07 \mathrm{dpm} \mathrm{m} \mathrm{m}^{-3}$ (6\% one sigma). Labs 1, 9, and 10 reported average ${ }^{230} \mathrm{Th}$ concentrations that deviated from the group mean by more than their reported analytical uncertainties, with average values for Labs 1 and 10 falling below the group mean and for Lab 9 falling above. Despite these differences, without eliminating any data the average ${ }^{230} \mathrm{Th}$ concentration reported by each lab fell within two sigma of the mean of all labs that reported results for the SAFe 3000-m sample. For ${ }^{231} \mathrm{~Pa}$ the situation is nearly as good, where the mean and standard deviation of lab mean values is $0.44 \pm 0.03 \mathrm{dpm} \mathrm{m}^{-3}$ (7\% one sigma) if one anomalously high value from Lab 2 and one low value from Lab 12 are excluded (Fig. 9B).

As noted above, the analysis of surface waters for ${ }^{230} \mathrm{Th}$ and ${ }^{231} \mathrm{~Pa}$ is challenging because of the low concentrations that are present, and this is reflected in the variability of ${ }^{230} \mathrm{Th}$ concentrations reported by Lab 2 and by the low ${ }^{231} \mathrm{~Pa}$ concentration reported by Lab 4 (Fig. 10). Nevertheless, excluding these results the generally good agreement among labs indicates that the low concentrations of these nuclides can be measured in surface waters and thereby contribute to the study of trace element scavenging and removal at shallow depths. Collection and analysis of replicate samples from surface waters are recommended to improve confidence in the results.

\section{Intercalibration-Particle analyses}

Determining concentrations of particulate ${ }^{230} \mathrm{Th},{ }^{232} \mathrm{Th}$, and ${ }^{231} \mathrm{~Pa}$ requires volumes of seawater greater than can be obtained using standard Niskin bottles because only a small fraction of the total $\mathrm{Th}$ and $\mathrm{Pa}$ in the open-ocean water column is associated with particulate material (compare results for filtered and unfiltered samples in Fig. 4). Consequently, as described above, particles were collected for intercalibration by filtering 400 to $600 \mathrm{~L}$ water using in situ pumps. Two locations were selected: (1) a depth of $2000 \mathrm{~m}$ at the BATS site, which allows particulate results to be compared with those from the intercalibration of dissolved nuclides (Fig. 7), and (2) at a depth of $80 \mathrm{~m}$ at a station on the continental slope $\left(37^{\circ}\right.$ $01.68^{\prime} \mathrm{N}, 74^{\circ} 23.88^{\prime} \mathrm{W}$ ). The slope site provided an opportunity for intercalibration in shallow water, where concentrations of dissolved ${ }^{230} \mathrm{Th}$ and ${ }^{231} \mathrm{~Pa}$ are much lower than in the deep open ocean, but where concentrations of particulate material are also likely to be much greater than in the open ocean. A one-quarter section of a filter from each site was distributed to each participating lab, along with a dip blank (see "Collection of particles" above for methods) from each site.

Five labs reported concentrations of particulate Th for the 2000-m samples at the BATS site while six labs reported concentrations of particulate ${ }^{231} \mathrm{~Pa}$ (Fig. 11). The average particulate ${ }^{230} \mathrm{Th}$ concentration at $2000 \mathrm{~m}$ is $\sim 0.05 \mathrm{dpm} \mathrm{m}^{-3}$ for four of the five reporting labs with Lab 9 reporting a substantially lower value (Fig. 11A). Investigators at the Woods Hole Oceanographic Institution determined that variability among subsamples from Supor filters deployed on different pumps, but sampling at the same time and location on the pump rosette, can be as large as 20\% (see "Collection of particles" above and Maiti et al. 2012). Therefore, the low value reported by Lab 9 may reflect a real difference between filter subsamples rather than an analytical bias. Setting aside the result from Lab 9, the average concentration of particulate ${ }^{230} \mathrm{Th}$ at $2000 \mathrm{~m}$ (Fig. 11A) is about $10 \%$ to $12 \%$ of the average concentration of dissolved ${ }^{230} \mathrm{Th}$ (Fig. 7C). This is somewhat less than, but nevertheless consistent with, the difference between filtered and unfiltered samples from $2000 \mathrm{~m}$ analyzed as part of the comparison of different filter membranes (estimated as $18 \%$ of ${ }^{230} \mathrm{Th}$ associated with filterable particles; see "Test of different filters" and Fig. 5C).

Given the potential for substantial heterogeneity within and between filters, it is informative to examine particulate ${ }^{232} \mathrm{Th} /{ }^{230} \mathrm{Th}$ ratios, which should be relatively insensitive to the heterogeneous distribution of particulate material over the surface of a filter provided that there is no fractionation by particle composition created by heterogeneous particle deposition on the membrane surface. We are aware of no evidence that such fractionation occurs. Here, in contrast to particulate Th concentrations, where the value reported by Lab 9 was low (Fig. 11A), we find that the ${ }^{232} \mathrm{Th} /{ }^{230} \mathrm{Th}$ ratio reported by Lab 9 is consistent with the results from three other labs, allowing for the possibility that the amount of particulate material 


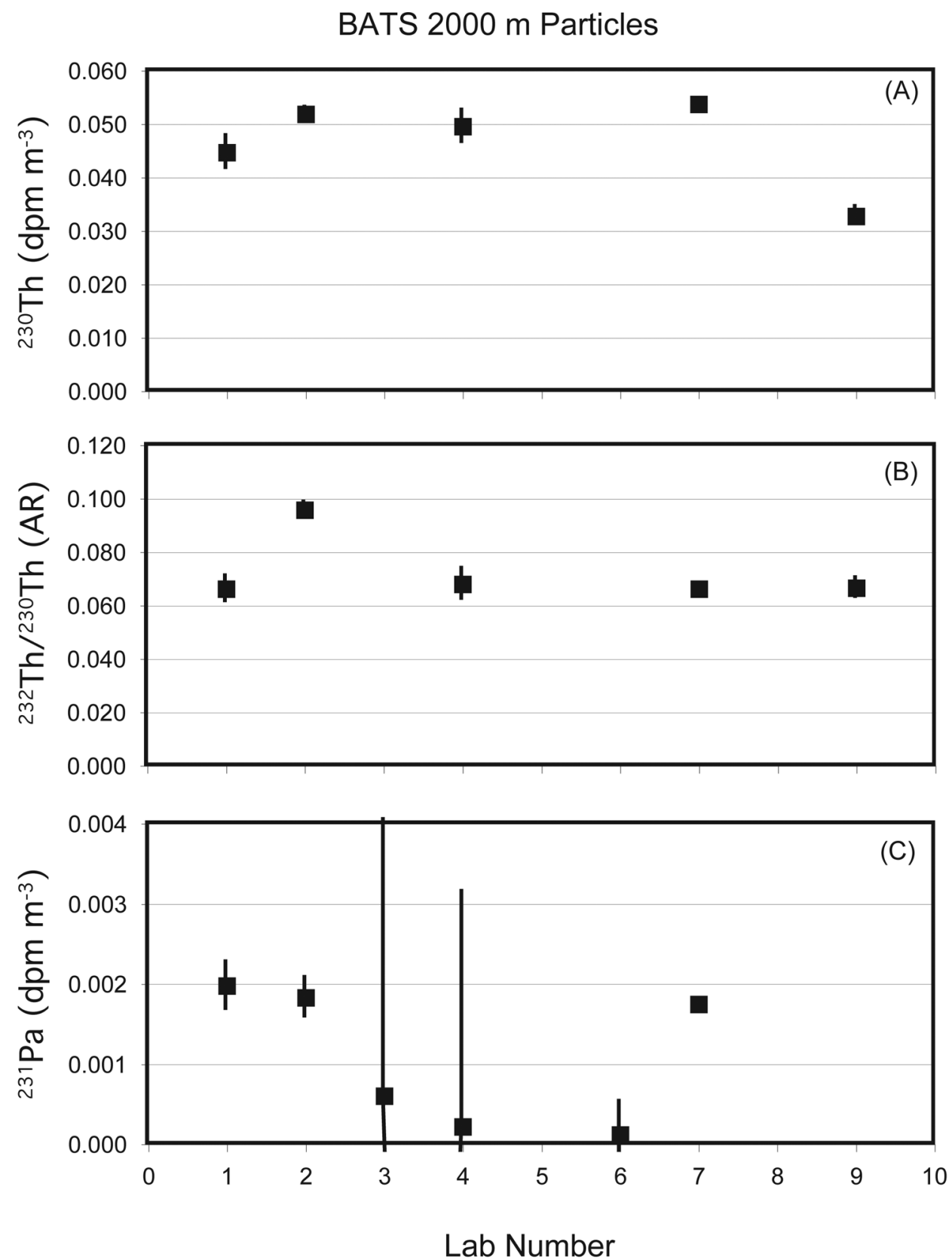

Fig. 11. Concentrations of $(A){ }^{230} \mathrm{Th}$ and $(\mathrm{C}){ }^{231} \mathrm{~Pa}$ in particulate matter collected by in situ filtration at a depth of $2000 \mathrm{~m}$ at the BATS site. (B) Particulate ${ }^{232} \mathrm{Th} /{ }^{230} \mathrm{Th}$ activity ratio. Lab numbers are unchanged from Fig. 6.

deposited on the section of filter analyzed by Lab 9 was below average. By contrast, the ${ }^{232} \mathrm{Th} /{ }^{230} \mathrm{Th}$ ratio reported by Lab 2 is greater than for the other four labs (Fig. 11B). Whether this reflects an unrecognized ${ }^{232} \mathrm{Th}$ blank or another factor affecting the results from Lab 2 is unknown.

Concentrations of particulate ${ }^{231} \mathrm{~Pa}$ were below the limit of detection for three of the six labs that reported results (Fig. 11C). Results from the remaining three labs are internally consistent and indicate a concentration of particulate
${ }^{231} \mathrm{~Pa}$ of about $0.0018 \mathrm{dpm} \mathrm{m} \mathrm{m}^{-3}$ (Fig. 11C), or about $1 \%$ of the concentration of dissolved ${ }^{231} \mathrm{~Pa}$ at the same location (Fig. 7C). These results are consistent with previous studies, which have determined that the fractionation factor representing the preferential scavenging of Th from seawater relative to $\mathrm{Pa}$, defined (Anderson et al. 1983b) as the ratio (particulate ${ }^{230} \mathrm{Th} /$ particulate $\left.{ }^{231} \mathrm{~Pa}\right) /\left(\right.$ dissolved ${ }^{230} \mathrm{Th} /$ dissolved ${ }^{231} \mathrm{~Pa}$ ), has a value of about 10 at $2000 \mathrm{~m}$ at the BATS site (Moran et al. 2002). 

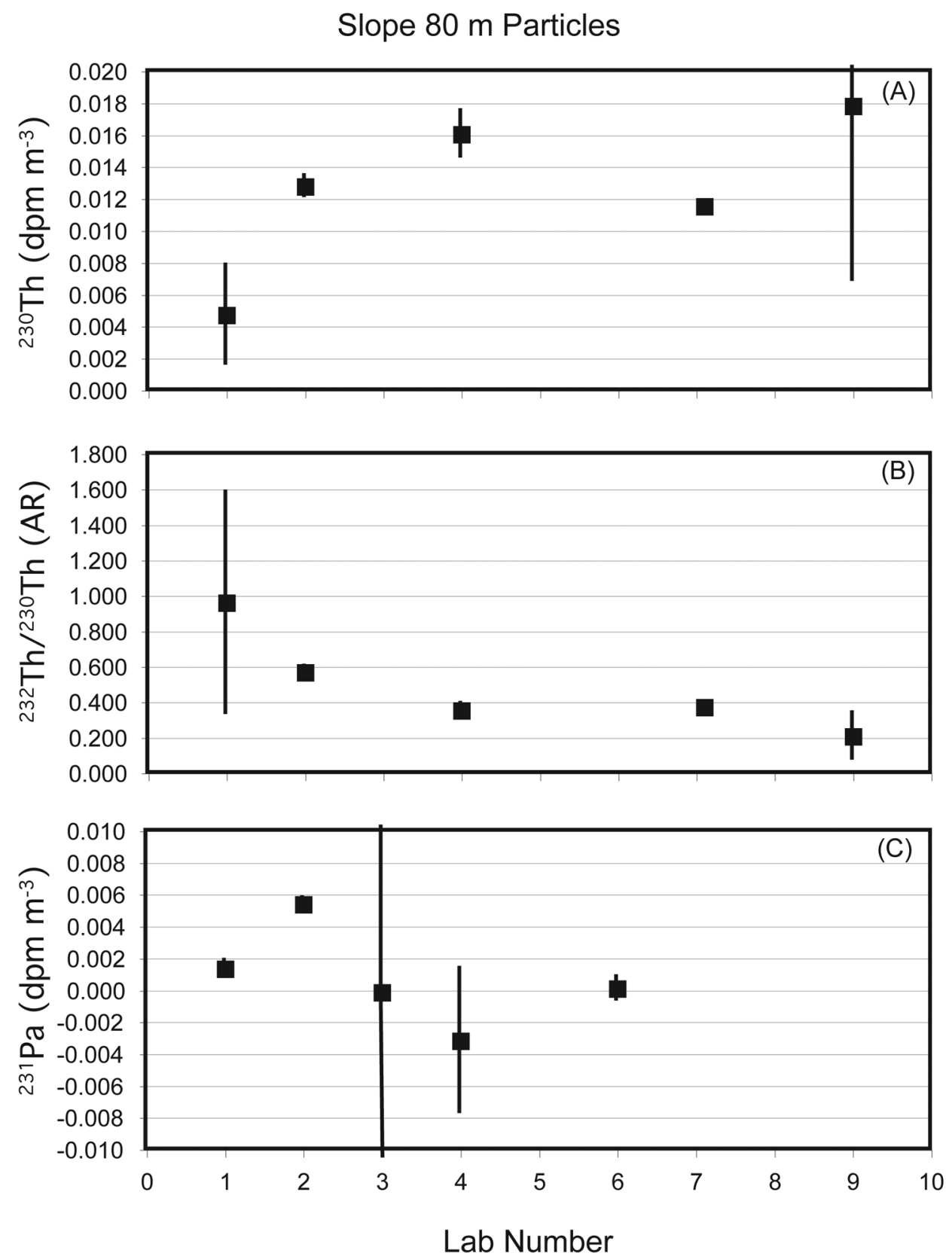

Fig. 12. Concentrations of $(A){ }^{230} \mathrm{Th}$ and $(C){ }^{231} \mathrm{~Pa}$ in particulate matter collected by in situ filtration at a depth of $80 \mathrm{~m}$ at a site over the continental slope off the east coast of the U.S. $\left(37^{\circ} 2.0^{\prime} \mathrm{N}, 74^{\circ} 23.87^{\prime} \mathrm{W}\right)$. (B) Particulate ${ }^{232} \mathrm{Th} /{ }^{230} \mathrm{Th}$ activity ratio. Note the position of "zero" on the concentration axis in (C). Lab numbers are unchanged from Fig. 6.

Concentrations of particulate ${ }^{230} \mathrm{Th}$ in surface waters at the slope station (Fig. 12A) are only about one quarter of the average reported for $2000 \mathrm{~m}$ at the BATS site (Fig. 11A), and the lower concentrations may contribute to the poorer reproducibility there among labs. In particular, the particulate ${ }^{230} \mathrm{Th}$ concentration reported by Lab 1 was only about one third of the average concentration reported by the other four labs, departing from the mean by more than can be attributed to heterogeneous particle collection ("Collection of particles").
The low particulate ${ }^{230} \mathrm{Th}$ concentration, together with the high particulate ${ }^{232} \mathrm{Th} /{ }^{230} \mathrm{Th}$ ratio reported by Lab 1 (Fig. 12B), suggests a problem with the ${ }^{230} \mathrm{Th}$. Five labs reported concentrations of particulate ${ }^{231} \mathrm{~Pa}$ for the shallow slope site. For three of five labs, the particulate ${ }^{231} \mathrm{~Pa}$ concentration was below their detection limit (note the position of zero concentration on the y-axis in Fig. 12C). For the two labs that reported particulate ${ }^{231} \mathrm{~Pa}$ concentrations significantly greater than their detection limits (Labs 1 and 2), the reported values differ by about a fac- 


\section{SW Standard 2010-1}
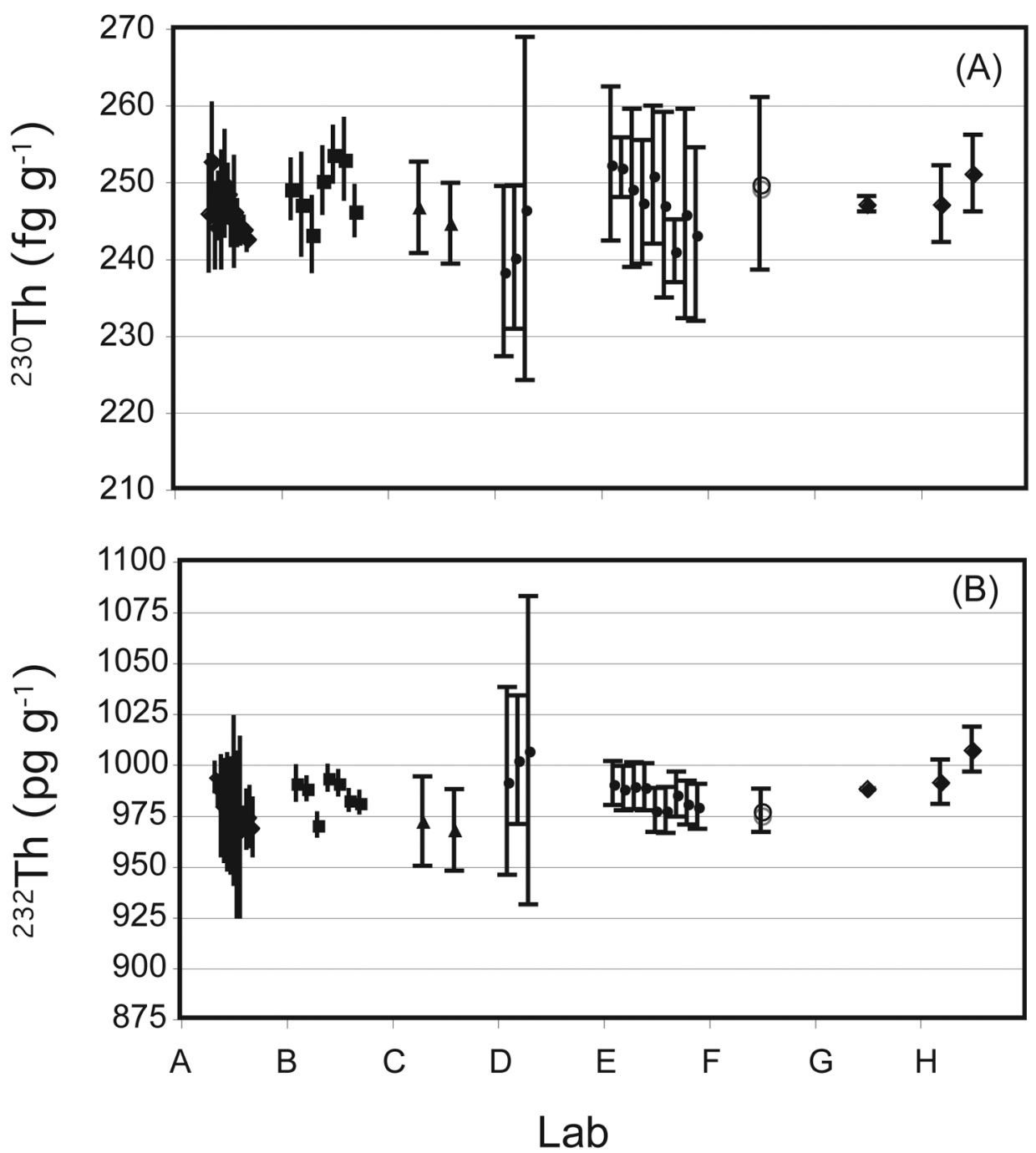

Fig. 13. Concentrations of (A) ${ }^{230}$ Th and (B) ${ }^{232}$ Th determined by analyzing aliquots of SW STD 2010-1 (see "Intercalibration-working standards") as an unknown. Units have been changed here to reflect the units used by participants. The following conversion factors are used at LDEO: ${ }^{232} \mathrm{Th}: 2.43438 \times$ $10^{-7} \mathrm{dpm} / \mathrm{pg} ;{ }^{230} \mathrm{Th} 0.04558 \mathrm{dpm} / \mathrm{pg}$. An insufficient number of results for ${ }^{231} \mathrm{~Pa}$ concentration have been reported to present them here. Labs are renamed here, and the sequence of labs differs from that in previous figures.

tor of three, too large to be attributable to sample heterogeneity. Determining concentrations of particulate ${ }^{230} \mathrm{Th}$ and ${ }^{231} \mathrm{~Pa}$ in surface waters is clearly challenging, and further work is needed to develop methods for the reliable determination of these concentrations at the low levels that occur in surface waters.

\section{Intercalibration-working standards}

Following discussion of the issues identified above, the participants determined that it would be beneficial to prepare and distribute a working solution that could be analyzed repeatedly to monitor the performance of each lab while also providing a further intercalibration of lab methods. A mixed solution was prepared at LDEO containing ${ }^{232} \mathrm{Th},{ }^{230} \mathrm{Th}$, and ${ }^{231} \mathrm{~Pa}$ in quantities such that $0.5 \mathrm{~g}$ of the solution contained approximately the same amount of each nuclide as would be present in $10 \mathrm{~L}$ seawater recovered from $2000 \mathrm{~m}$ at the BATS site. The solution was then split into two aliquots, and ${ }^{229} \mathrm{Th}$ was added to one of the two portions. The portion without ${ }^{229} \mathrm{Th}$, labeled SW STD 2010-1, was to be treated as an unknown and processed as for any other intercalibration. Each lab was asked to add its own yield monitors $\left({ }^{233} \mathrm{~Pa}\right.$ and $\left.{ }^{229} \mathrm{Th}\right)$ and process the standard through normal chemical procedures. Comparison of results was expected to yield information about overall consistency among labs, including blanks and the calibration of yield monitors. Participants were asked to process the portion containing ${ }^{229} \mathrm{Th}$ (labeled SW STD 2010-2) without adding 
SW Standard 2010-2 Atom Ratios
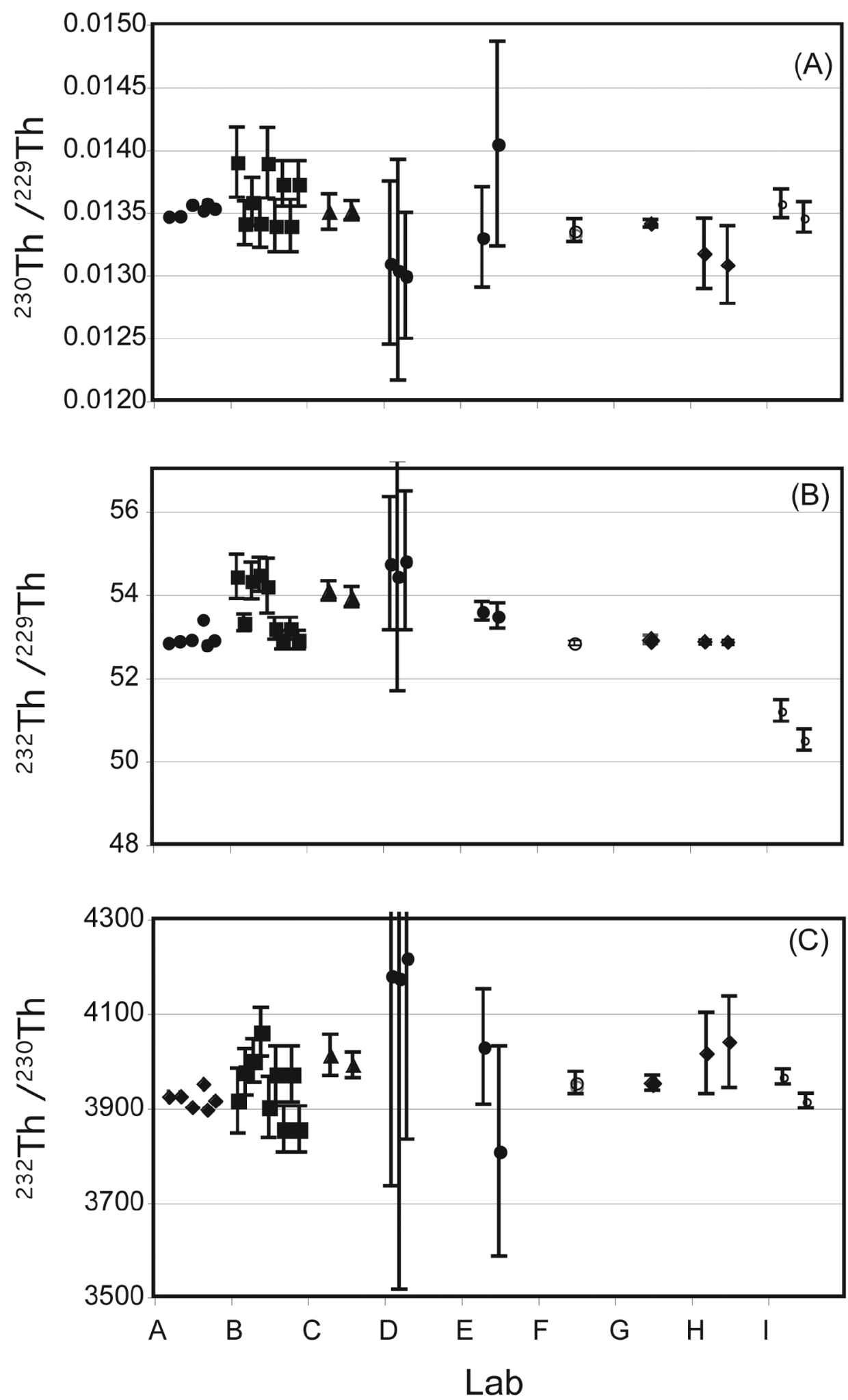

Fig. 14. Atom ratios reported by participating labs for SW STD 2010-2, the solution that had been spiked with ${ }^{229}$ Th at LDEO (see "Intercalibration-working standards"). Labs identifications (A-H) are the same as in Fig. 13. Lab I reported Th atom ratios but not Th concentrations (Fig. 13). 
yield monitors to determine Th atom ratios in the solution as a measure primarily of the mass biases inherent in mass spectrometers, although blanks would affect these ratios as well.

Different procedures were used among the labs that have submitted results for the working solutions. At one extreme, SW STD 2010-1 was added to several liters of distilled water that was then processed as a full seawater sample. At the other extreme, the analyst simply spiked a weighed aliquot of the SW STD 2010-1 solution, which was then evaporated, reconstituted and injected directly into the mass spectrometer. Results are reported in Fig. 13 without distinction among the types of procedures applied. Also, participating labs have been renamed here. That is, Lab A in the results for the working solutions is not Lab 1 in the results for seawater, sediments, and particles, and so forth.

Among the eight labs that have submitted results to date for SW STD 2010-1, concentrations of ${ }^{230} \mathrm{Th}$ agreed within the uncertainties reported by each lab (Fig. 13A). The same is true for ${ }^{232} \mathrm{Th}$ (Fig. 13B) although it would be desirable to have more results from each lab to assess the overall internal consistency. For ${ }^{230} \mathrm{Th}$, the average and standard deviation of the lab mean values is $247 \pm 3 \mathrm{pg} / \mathrm{g}$, from which it can be inferred that the calibration of yield monitors for Th is internally consistent among labs to within about $1 \%$. In principle, if other factors (e.g., blanks) can be controlled, then it should be possible to constrain global variability in the concentration of ${ }^{230} \mathrm{Th}$ at nearly this level of precision.

In light of the good agreement among labs that reported concentrations of ${ }^{230} \mathrm{Th}$ and ${ }^{232} \mathrm{Th}$ for SW STD 2010-1, it is surprising that labs differ by as much as they do in results for SW
STD 2010-2 (pre-spiked with ${ }^{229} \mathrm{Th}$ ). The largest reported uncertainties were experienced for ${ }^{230} \mathrm{Th} /{ }^{229} \mathrm{Th}$ (Fig. 14A) and ${ }^{232} \mathrm{Th} /{ }^{230} \mathrm{Th}$ (Fig. 14C) ratios because ${ }^{230} \mathrm{Th}$ was present in the lowest abundance among the three Th isotopes. Differences between labs for these ratios are just at the limit of exceeding reported uncertainties, so it is difficult to establish their significance. Nevertheless, among the nine labs reporting results for SW STD 2010-2 the mean and standard deviation of lab mean ${ }^{230} \mathrm{Th} /{ }^{229} \mathrm{Th}$ atom ratios is $0.01338 \pm 0.00022(1.6 \% 1$ sigma). Finding a slightly poorer reproducibility here than for the determination of the ${ }^{230}$ Th concentration in SW STD 20101 (247 $\pm 3 \mathrm{pg} / \mathrm{g}$, see above) points to blanks and instrumental errors rather than spike calibration as the current factor limiting interlab reproducibility in determining the concentration of ${ }^{230} \mathrm{Th}$.

By contrast, ratios reported for the two more abundant isotopes $\left({ }^{232} \mathrm{Th} /{ }^{229} \mathrm{Th}\right)$ differ by more than the reported uncertainties (Fig. 14B). It appears as if there are two clusters of ${ }^{232} \mathrm{Th} /{ }^{229} \mathrm{Th}$ ratios, with results from Labs $\mathrm{A}, \mathrm{F}, \mathrm{G}$, and $\mathrm{H}$ falling about the lower value and those from Labs B, C, and D falling about the higher value. Lab I reported ${ }^{232} \mathrm{Th} /{ }^{229} \mathrm{Th}$ ratios significantly below the range of values reported by other labs, even though the ${ }^{230} \mathrm{Th} /{ }^{229} \mathrm{Th}$ and ${ }^{232} \mathrm{Th} /{ }^{230} \mathrm{Th}$ ratios from Lab I are consistent with those from other labs. The source of the disagreement remains undetermined. Lab B reported a large number of results, among which the ${ }^{232} \mathrm{Th} /{ }^{229} \mathrm{Th}$ ratios seem to fall within one or the other of the clusters defined by seven of the other labs (excluding Lab I). Keeping in mind that each lab was running the same solution with the same ${ }^{232} \mathrm{Th} /{ }^{229} \mathrm{Th}$ ratio, the differences reported here are interpreted to indicate

\section{BATS 2000 m Seawater}

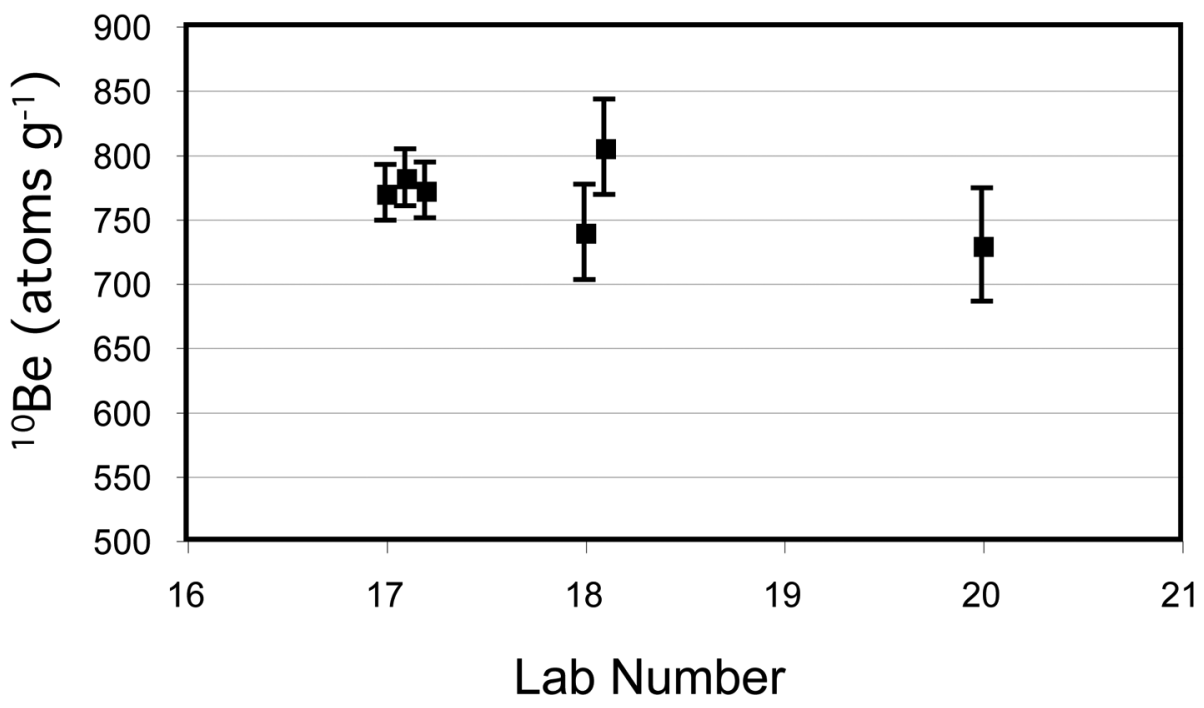

Fig. 15. Concentrations of ${ }^{10} \mathrm{Be}$ determined by analyzing aliquots of homogenized seawater collected from a depth of $2000 \mathrm{~m}$ at the BATS site. Samples were drawn from the 1000-L SAFe tank system that had been filled using repeated casts of the U.S. GEOTRACES carousel, equipped with GO-Flo bottles. Water was filtered as it was transferred from the GO-Flo bottles to the tanks and acidified to a pH 1.7. 
instrumental bias in the reported atom ratios. The differences appear to be too large to have been caused by a mass bias issue, but may reflect a problem with the calibration of the efficiency of the ion counter relative to the Faraday cups on certain instruments. There is no correlation with the type of mass spectrometer employed (TIMS versus ICP-MS). Whatever its origin, the consistency among labs for the Th concentrations reported for SW STD 2010-1 (Fig. 13) suggests that any instrument bias in atom ratios cancels out when the same bias is first applied to calibration of the ${ }^{229} \mathrm{Th}$ yield monitor used by each lab, and later the yield monitor is used to determine the concentrations of ${ }^{232} \mathrm{Th}$ and ${ }^{230} \mathrm{Th}$ in an unknown.

We note that the ${ }^{229} \mathrm{Th}$ content of SW STD 2010-2 was greater than the amount that some labs typically use when spiking seawater samples. Consequently, some instruments may have had trouble with the large range in intensities compared with normal efforts for a well-matched ${ }^{230} \mathrm{Th} /{ }^{229} \mathrm{Th}$ ratio in spiked samples.

\section{Intercalibration $-{ }^{10} \mathrm{Be}$ in seawater}

Beryllium-10 was included with this group for intercalibration because, historically, ${ }^{10} \mathrm{Be}$ concentrations in seawater and in sediments have been determined by many of the same labs that determine concentrations of $\mathrm{Th}$ and $\mathrm{Pa}$. Furthermore, it is hoped that it will be possible at some point in the future to use the spatial variability in the ratio of dissolved ${ }^{10} \mathrm{Be}$ (cosmogenic) to ${ }^{9} \mathrm{Be}$ (lithogenic) to constrain rates of mixing in the ocean. In light of this anticipated application that will be of interest to the GEOTRACES community, we present intercalibration results for dissolved ${ }^{10} \mathrm{Be}$ even though only three labs have reported. Seawater from $2000 \mathrm{~m}$ at the BATS site was distributed for intercalibration of ${ }^{10} \mathrm{Be}$. Results to date are internally consistent within the uncertainties reported by each lab
(Fig. 15). The fraction of ${ }^{10} \mathrm{Be}$ in seawater that is associated with filterable particles is expected to be much less than for ${ }^{231} \mathrm{~Pa}$ (i.e., $<<1 \%$; see "Discussion of issues"), so no attempt was made to measure ${ }^{10} \mathrm{Be}$ concentrations in particulate material recovered by in situ filtration.

\section{Baseline station-BATS}

One of the objectives of the GEOTRACES intercalibration initiative is to establish "baseline stations" where concentration profiles of trace elements and their isotopes will be determined by several laboratories repeatedly throughout the duration of the program. Repeated analyses at these stations provide an ongoing measure of intercalibration. Baseline stations also provide a location where any lab can collect samples and expect that the concentrations of trace elements or isotopes are known reasonably well, at least in the deep ocean where concentrations are not expected to change significantly on decadal time scales. Here we illustrate this principle using concentrations of ${ }^{230} \mathrm{Th}$ and of ${ }^{232} \mathrm{Th}$ measured at the BATS site. In the next section, we describe evidence from the Pacific to illustrate some caveats concerning the baseline station concept.

Three of the labs participating in the intercalibration of Th and $\mathrm{Pa}$ have generated concentration profiles of ${ }^{230} \mathrm{Th}$ and of ${ }^{232} \mathrm{Th}$ at the BATS site over a period of 16 years. Duplicate profiles (i.e., beginning with separate Niskin bottles and processing samples independently) collected during KN193-6 (July 2008) and analyzed at LDEO were filtered at the time of collection through $0.45 \mu \mathrm{m}$ pore size Supor filters, like those used in the filtration tests (e.g., Fig. 4). The profiles analyzed by the other two labs were processed without filtration, so this difference in processing must be considered when comparing results. Two-liter samples of unfiltered seawater collected on 7 July 1993 during the R/V Weatherbird cruise 93-06 were ana-
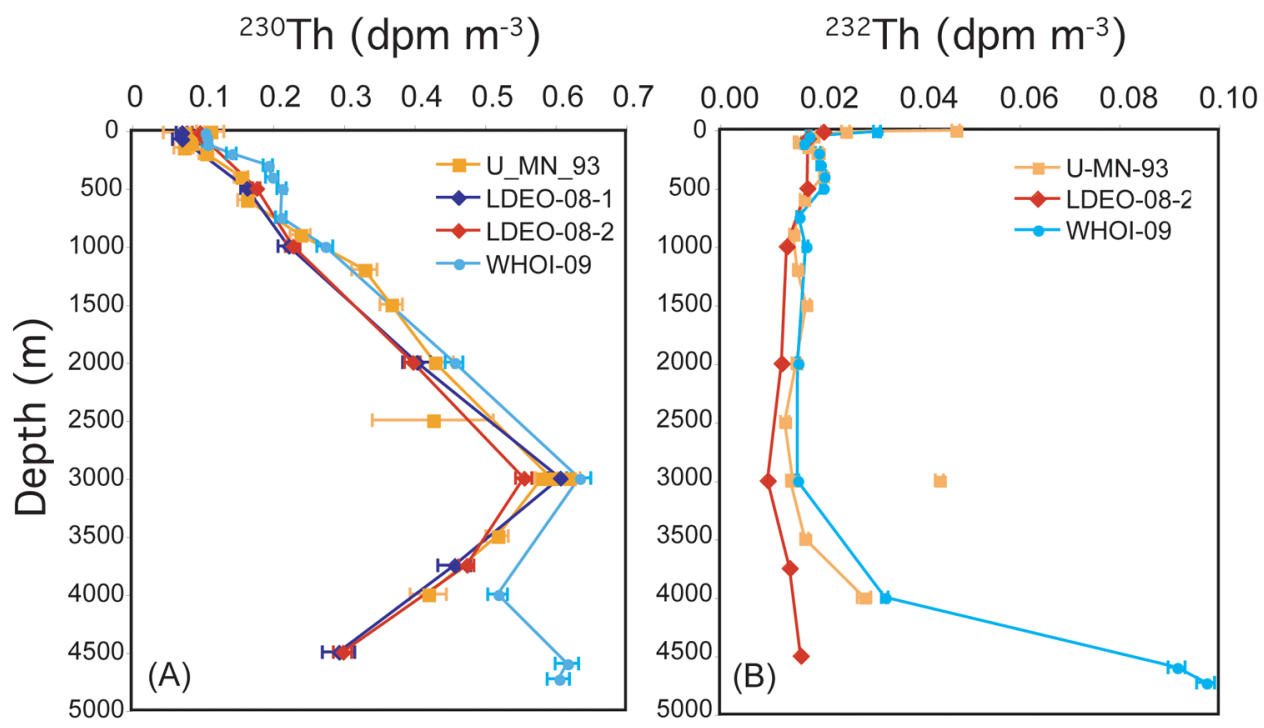

Fig. 16. Baseline concentration profiles for ${ }^{230} \mathrm{Th}(\mathrm{A})$ and ${ }^{232} \mathrm{Th}(\mathrm{B})$ at the BATS site (roughly $75 \mathrm{~km}$ southeast of Bermuda at $31^{\circ} 50^{\prime} \mathrm{N}, 64^{\circ} 10^{\prime} \mathrm{W}$ ). Unfiltered samples collected in 1993 were analyzed at the University of Minnesota (see "Minnesota chemical procedures"). Unfiltered samples collected in 2009 were analyzed at the Woods Hole Oceanographic Institution following methods described in Auro et al. (2012). Duplicate samples of filtered seawater were collected during KN193-6 in 2008 aboard the first GEOTRACES intercalibration cruise and analyzed at LDEO (see "Collection of seawater"). 
lyzed shortly after collection at the University of Minnesota using TIMS. Samples from $>200 \mathrm{~m}$ were collected in $10 \mathrm{~L}$ Niskin bottles using a CTD/Rosette deployed on a steel hydrowire. Samples from < $200 \mathrm{~m}$ were collected either using GO-Flo bottles suspended on a stainless steel wire or on a separate cast using trace metal clean bottles suspended on a Kevlar line provided by Dr. Jim Moffet (WHOI). The second set of unfiltered samples was collected in September 2009 by Ken Buesseler on cruise AE0908 of the R/V Atlantic Explorer. Samples were collected via Masterflex Tygon tubing directly from the Niskins into cubitainers. Each 10-L cubitainer was rinsed with $\sim 50 \mathrm{~mL}$ seawater then filled. Optima grade $\mathrm{HCl}(1 \mathrm{~mL}$ concentrated $\mathrm{HCl}$ per liter seawater) was added to each sample in a clean hood.

In each profile, the concentration of ${ }^{230} \mathrm{Th}$ increases with depth from the surface to $\sim 3000 \mathrm{~m}$. Below $\sim 3000 \mathrm{~m}$, the concentration of dissolved ${ }^{230} \mathrm{Th}$ decreases toward the bottom (Fig. 16A), as is often observed in the North and South Atlantic Ocean (Luo et al. 2010; Moran et al. 2002). Concentrations of total ${ }^{230} \mathrm{Th}$ in the two sets of unfiltered samples (U-MN and WHOI) are in good agreement above $3000 \mathrm{~m}$ despite the 16 years between collection of the two sets of samples. This agreement illustrates the principle that baseline stations can be used to provide access to seawater with known concentrations of trace elements and their isotopes in stable regions of the ocean interior (but see caveats below). Concentrations of dissolved ${ }^{230}$ Th (Fig. 16A; LDEO) exhibit good agreement between repli- cate profiles, and they are slightly but systematically lower than concentrations in the unfiltered samples (Fig. 16A; U-MN and WHOI). This is to be expected given that about $10 \%$ of the total ${ }^{230} \mathrm{Th}$ in mid-depth waters resides on particles that can be removed by filtration (see "Test of different filters").

Concentrations of total ${ }^{232} \mathrm{Th}$ agree well between the two sets of unfiltered samples (WHOI and U-MN), and the total ${ }^{232} \mathrm{Th}$ concentration is uniformly greater than the dissolved $\left(<0.45 \mu \mathrm{m}\right.$; LDEO) ${ }^{232} \mathrm{Th}$ concentration throughout the water column (Fig. 16B). The first set of replicate samples from KN193-6 (LDEO) experienced high ${ }^{232}$ Th blanks, so the results are not reported here. Below $3000 \mathrm{~m}$, the profiles of filtered and unfiltered samples diverge, more so for ${ }^{232} \mathrm{Th}$ than for ${ }^{230} \mathrm{Th}$ (Fig. 16). We interpret this to indicate the presence of resuspended sediment particles throughout the lower $\sim 2 \mathrm{~km}$ of the water column, which increases the concentration of total Th relative to mid-water column depths. The presence of resuspended sediments is supported by transmissometer profiles collected during KN193-6, which show beam transmission decreasing below $\sim 3740 \mathrm{~m}$ at the BATS site (not shown). The abundance of resuspended sediments may vary with time, a factor that should be taken into account when interpreting Th concentrations in near-bottom waters from the BATS site.

Concentrations of ${ }^{230} \mathrm{Th}$ and of ${ }^{232} \mathrm{Th}$ measured as part of the baseline profile, both with and without filtration (Fig. 16), are consistent with the concentrations reported for filtered and unfiltered samples as part of the test of different filter

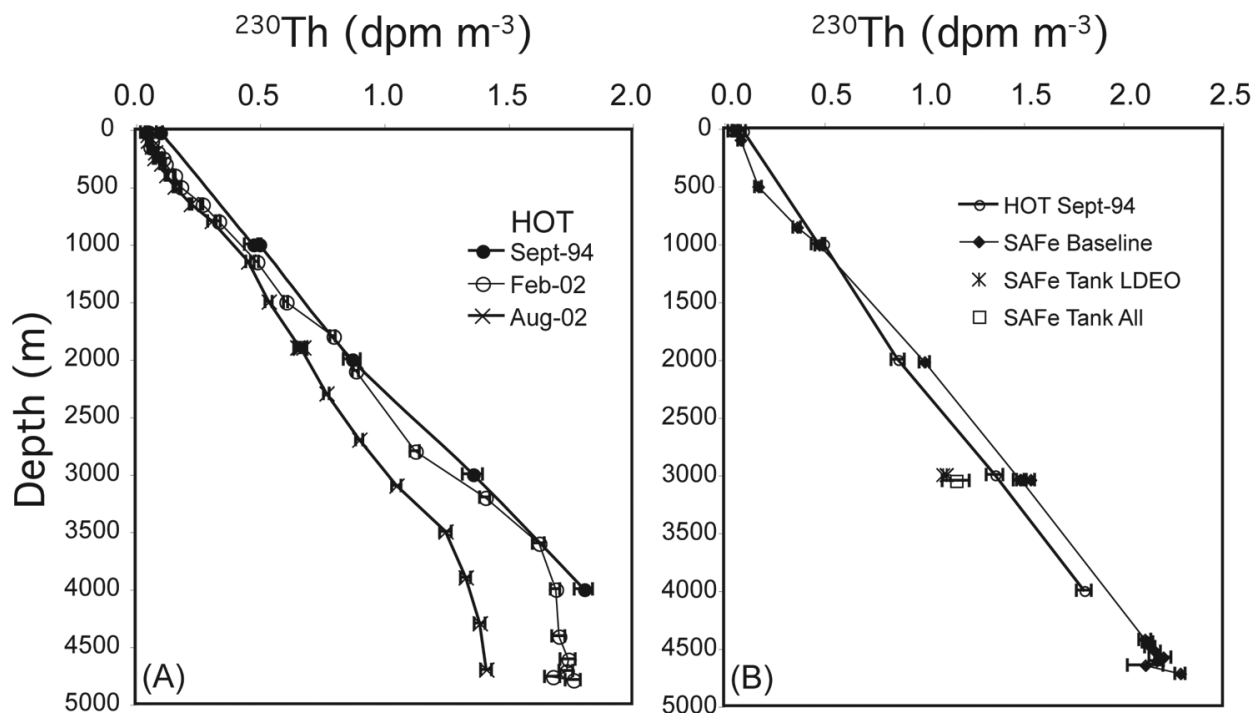

Fig. 17. (A) Concentration profiles for ${ }^{230} \mathrm{Th}$ collected at the Hawaii Ocean Time Series (HOT) Aloha station $\left(22^{\circ} 45^{\prime} \mathrm{N}, 158^{\circ} \mathrm{W}\right)$. Samples collected in 1994 were acidified ( $\sim 2 \mathrm{~mL}$ doubly distilled $\mathrm{HCl}$ per liter of seawater) without filtration (Roy-Barman et al. 1996). Samples collected in 2002 were processed as described by Choi et al. (2001). (B) Results for ${ }^{230} \mathrm{Th}$ at the SAFe Station $\left(40^{\circ} \mathrm{N}, 140^{\circ} \mathrm{W}\right)$, based on samples collected during the second GEOTRACES intercalibration cruise, are compared with the September 1994 results from the HOT site. SAFe baseline profile samples were collected in Niskin bottles and gravity filtered through Pall Acropak ${ }^{\mathrm{TM}} 500$ filters, which contain a paired, pleated filter arrangement containing a $0.8 \mu \mathrm{m}$ pre-filter followed by a $0.45 \mu \mathrm{m}$ filter. Filtered samples were acidified with $4 \mathrm{~mL} / \mathrm{L}$ of ultraclean $6 \mathrm{~N} \mathrm{HCl}$ to bring the solution to a $\mathrm{pH}$ of $\sim 1.7$. SAFe tank results are from Fig. 9. Triplicate results from SAFe tank samples processed at LDEO are shown as individual points. Results from all other labs (Fig. 9) are presented as a grand average (SAFe Tank All) and one standard deviation of the mean values reported by labs that reported results. The depth of the grand average is offset from the LDEO results for clarity. Triplicate results from the baseline profile at $3042 \mathrm{~m}$ represent the full procedural analysis of three individual samples. 
membranes (Fig. 4). They are also consistent with the concentrations of dissolved (Fig. 7C) and particulate (Fig. 11A) ${ }^{230} \mathrm{Th}$ determined through the intercalibration exercise, with the caveat that there was substantial variability among participating labs in the results that were reported. Based on the internal consistency among results from different labs and from samples collected at different times, we conclude that middepth water at the BATS site can serve as a baseline station for ongoing intercalibration of ${ }^{230} \mathrm{Th}$ and ${ }^{232} \mathrm{Th}$.

\section{Baseline station-Pacific}

Results from two locations in the central north Pacific Ocean suggest that the assumption of constant or known nuclide concentrations in deep waters may not always be valid. Results from samples collected in 1994 at the Hawaii Ocean Time Series (HOT) Station Aloha $\left(22^{\circ} 45^{\prime} \mathrm{N}, 158^{\circ} \mathrm{W}\right)$ show the concentration of ${ }^{230} \mathrm{Th}$ increasing linearly with depth (Fig. 17A, Roy-Barman et al. 1996), as expected for removal by reversible scavenging to sinking particles (Bacon and Anderson 1982). Concentrations of dissolved ${ }^{230} \mathrm{Th}$ derived from samples collected at the HOT site in February 2002 agree well with the results from samples collected in 1994 (Fig. 17A, Francois 2007). However, the concentration profile of ${ }^{230} \mathrm{Th}$ determined from samples collected during a reoccupation of Station Aloha 6 months later, analyzed by the same lab, and using the same methods as for the February 2002 samples depart significantly from results obtained during two previous occupations (Fig. 17A, R. Francois unpubl. data). Surprisingly, whereas concentrations of ${ }^{230} \mathrm{Th}$ in the upper $1000 \mathrm{~m}$ of the water column agree within error for the two occupations in 2002 , results diverge with increasing water depth, differing by nearly $20 \%$ for the deepest samples. It is unlikely that nearly $20 \%$ of the ${ }^{230} \mathrm{Th}$ in deep water could have been removed by scavenging over an interval of about 6 months, as this would imply removal of ${ }^{230} \mathrm{Th}$ much faster than is inferred from its overall residence time of several decades. Whether the divergence of deepwater results between the February and August 2002 occupations reflects the movement of a deep water mass with lower ${ }^{230} \mathrm{Th}$ concentration into the vicinity of the Aloha station or some other factor remains to be determined.

A similarly disconcerting finding is evident in results from the second GEOTRACES intercalibration cruise at the SAFe site. There, the concentration of dissolved ${ }^{230} \mathrm{Th}$ increases linearly with depth, much as in the 1994 results from the Aloha station (Fig. 17B). However, concentrations of dissolved ${ }^{230} \mathrm{Th}$ determined using samples from the 3000-m SAFe tank (Fig. 9) fall between $20 \%$ and $25 \%$ below the value from an equivalent depth in the profile obtained using Niskin bottles (Fig. 17B). Triplicate samples from the SAFe tank $(3000 \mathrm{~m})$ and triplicate Niskin samples (3042 m) were each processed at LDEO using the same spike and the same analytical procedure. Internal consistency among each set of samples indicates that random contamination was not responsible for the systematic difference. Although the SAFe tank samples were processed through filters with a smaller effective pore diameter $(0.22 \mu \mathrm{m})$ than the Niskin samples $(0.45 \mu \mathrm{m})$, tests at the BATS site indicate that differences this large would not be expected for the different filtration procedures (Fig. 4). Niskin-profile and SAFetank samples were collected within a few days of one another at the same location. Therefore, neither scavenging nor displacement of deep water masses can be invoked to explain the divergent results. The cause of this difference remains unresolved. Future tests of filter pore diameter should be conducted to establish the abundance of colloidal $(<0.45 \mu \mathrm{m}) \mathrm{Th}$ and the sensitivity of dissolved ${ }^{230} \mathrm{Th}$ concentrations to filter pore size.

Good agreement between results from samples collected in 1994 and in February 2002 at the HOT site provides reason for optimism that different labs, using different methods, can obtain consistent results over a period of more than a decade. However, results from the two occupations of the HOT site in 2002 indicate that concentration of ${ }^{230} \mathrm{Th}$ in the deep ocean may vary on subannual time scales by nearly $20 \%$. Results from the SAFe site also indicate that factors still to be identified may have a substantial (20\% to $25 \%$ ) effect on reported ${ }^{230}$ Th concentrations. Additional tests will be required to determine whether these factors involve unknown analytical artifacts. Whether or not such artifacts exist, the results from the HOT site offer reason to suspect that nature may not be as stable as thought; that is, that concentrations of nuclides in the deep ocean cannot be assumed to remain constant over multidecadal time scales in some locations. If so, then another strategy for sustained intercalibration must be sought. Further discussion among the community making these measurements will determine whether archived aliquots of homogenized seawater samples, such as those collected from the SAFe tank (see above), or artificial seawater standards (Fig. 13), are best suited for this purpose.

\section{Prior intercalibration efforts}

Before GEOTRACES effort, the author of this manuscript from the Alfred Wegener Institute (Bremerhaven, Germany) initiated an intercalibration of labs that measure $\mathrm{Th}$ and $\mathrm{Pa}$ in seawater. Two sets of samples were distributed. The first set was collected aboard the R/V Polarstern from $2000 \mathrm{~m}$ depth in the Angola Basin on 9 Nov 2005 at station PS69/021 $\left(11^{\circ} 51.80^{\prime} \mathrm{S}, 2^{\circ} 30.72^{\prime} \mathrm{W}\right)$. Unfiltered water, collected with a standard rosette, was acidified with $1 \mathrm{~mL} / \mathrm{L}$ quartz-distilled $\mathrm{HCl}$. A second set of samples was collected from a depth of $2000 \mathrm{~m}$ on 2 Sep 2007 aboard the R/V Polarstern during cruise ARK XXII/2 in the Makarov Basin (Arctic Ocean). Two subsets of Arctic samples were collected, one using the standard ship's rosette and one using the trace metal-clean sampling system of the Netherlands Institute of Sea Research (De Baar et al. 2008). Unfiltered Arctic samples were acidified with $1 \mathrm{~mL} / \mathrm{L}$ Seastar concentrated $\mathrm{HNO}_{3}$.

Results from both sets of samples are reported together in Fig. 18 because of the relatively small number of data. Reporting the results together is not meant to imply that concentrations of Th and $\mathrm{Pa}$ in the Angola Basin are expected to be the 


\section{Angola (Atlantic) and Makarov (Arctic) Basin Seawater}
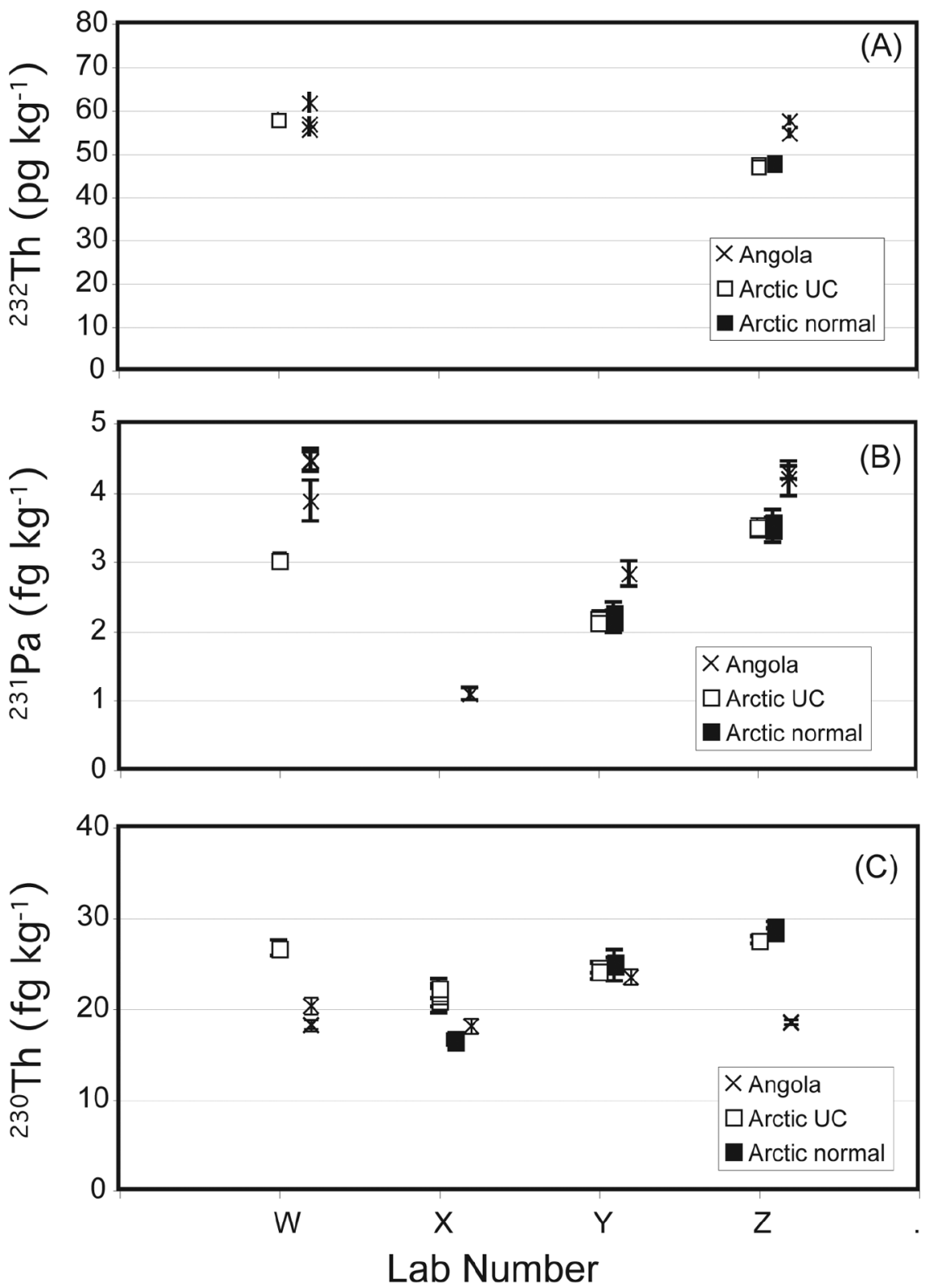

Fig. 18. Concentrations of $(A){ }^{232} \mathrm{Th},(B){ }^{231} \mathrm{~Pa}$, and $(\mathrm{C}){ }^{230} \mathrm{Th}$ determined by analyzing samples of seawater collected from the Angola Basin and from the Arctic Ocean (see "Prior intercalibration efforts" for collection details). Unfiltered samples were acidified at sea. Samples from the Angola Basin and those labeled "Arctic normal" were collected using a standard rosette. Samples labeled "Arctic UC" were collected using the trace metal-clean sampling system of the Netherlands Institute of Sea Research (De Baar et al. 2008). Lab identifications in this figure differ from those in previous figures, but Labs W-Z here are represented among Labs 1-12 in Figs. 6-9.

same as in the Arctic Ocean. Only two labs (Labs W and Z) reported concentrations of ${ }^{232} \mathrm{Th}$ (Fig. 18A). Values reported by Labs $\mathrm{W}$ and $\mathrm{Z}$ are in agreement for the Angola Basin samples, indicating that there was no bias associated with spike calibration. Therefore, it is unclear why the concentration of ${ }^{232} \mathrm{Th}$ reported by Lab $\mathrm{Z}$ for the Arctic Ocean is significantly less than that reported by Lab W. In light of the widespread but variable contamination problems encountered for ${ }^{232} \mathrm{Th}$ during the GEOTRACES intercalibration (see "Intercalibration-Seawater analyses"), contamination may have been a factor. The average concentration of ${ }^{230} \mathrm{Th}$ reported for the Angola Basin is $19.7 \mathrm{fg} / \mathrm{kg}$ (Fig. 18C). Only Lab W reported values within their reported uncertainty of the overall average. Concentrations reported by other labs differed from the mean by $10 \%$ to $15 \%$. 
Concentrations of ${ }^{231} \mathrm{~Pa}$ reported for Angola Basin samples range over a factor of four (Fig. 18B), indicating a problem with the determination of this nuclide. Concentrations reported by Labs $\mathrm{W}$ and $\mathrm{Z}$ agree to within reported uncertainty while values from Labs $\mathrm{X}$ and $\mathrm{Y}$ are much lower than those reported by Labs $\mathrm{W}$ and $\mathrm{Z}$.

As for the Angola Basin, Labs $\mathrm{W}$ and $\mathrm{Z}$ reported concentrations of ${ }^{230} \mathrm{Th}$ and ${ }^{231} \mathrm{~Pa}$ for the Arctic samples that either agree within the reported uncertainties, or differ by slightly more than the reported uncertainties. Concentrations of ${ }^{230} \mathrm{Th}$ and ${ }^{231} \mathrm{~Pa}$ reported by Lab Y were lower than values reported by Labs $\mathrm{W}$ and $\mathrm{Z}$. The difference was slightly greater than the uncertainty reported by Lab Y for ${ }^{230} \mathrm{Th}$ (Fig. 18C), but much greater for ${ }^{231} \mathrm{~Pa}$ (Fig. 18B). Lab $\mathrm{X}$ did not report a ${ }^{231} \mathrm{~Pa}$ result for the Arctic samples and reported a concentration of ${ }^{230} \mathrm{Th}$ below those of Labs W, Y, and Z.

These results indicate that problems existed among some labs measuring Th and $\mathrm{Pa}$ in seawater before the GEOTRACES intercalibration was implemented. One encouraging result from the pre-GEOTRACES intercalibration is that with one exception ( $\mathrm{Lab} \mathrm{X},{ }^{230} \mathrm{Th}$ ), there is no significant difference between results from the standard rosette and those from the trace metal-clean system where samples from the two systems were analyzed by the same lab. This is consistent with the conclusion from the GEOTRACES intercalibration that seawater can be collected without contamination for Th and $\mathrm{Pa}$ using standard rosettes (see "Niskin vs. GO-Flo").

\section{Discussion}

The GEOTRACES intercalibration brought together many members of the international community of investigators who determine the concentrations of long-lived radionuclides in marine samples, many of whom had had no prior interaction. This community-building effort will facilitate future collaboration in methods development as well as in research on marine processes. Results from the analysis of marine sediments (Fig. 6) represent the largest effort to date to establish the internal consistency among labs that determine ${ }^{232} \mathrm{Th}$, ${ }^{230} \mathrm{Th}$, and ${ }^{231} \mathrm{~Pa}$ concentrations in marine samples. Despite the evidence for some heterogeneity of the sediment, with a few exceptions the results indicated good agreement among the participating labs.

Results from the first GEOTRACES intercalibration cruise (Figs. 7, 8, 11, 12) indicated that some labs were unable to determine concentrations of dissolved and particulate ${ }^{232} \mathrm{Th}$, ${ }^{230} \mathrm{Th}$, and ${ }^{231} \mathrm{~Pa}$ with sufficient accuracy and precision to undertake a global survey of the distribution of these nuclides in the ocean. Poor reproducibility was also evident in the results from a previous intercalibration among four of the labs that later participated in the GEOTRACES intercalibration (Fig. 18).

A substantial improvement in the performance of the participating labs was evident in the results reported over the course of the GEOTRACES intercalibration initiative, illustrating the benefits of such intercalibrations to the marine geo- chemistry community. By the time of the second intercalibration cruise, many labs had made progress in reducing their ${ }^{232}$ Th blanks, although others were still working on the problem. Results from the second cruise demonstrated that participating labs can determine concentrations of dissolved ${ }^{230} \mathrm{Th}$ and ${ }^{231} \mathrm{~Pa}$ in the deep sea that are internally consistent to within $7 \%$ of the mean value (Fig. 9). Results from eight labs that analyzed a solution containing unknown concentrations of ${ }^{232} \mathrm{Th}$ and ${ }^{230} \mathrm{Th}$ indicate that calibration of Th isotope yield monitors is consistent among the labs to within about $1 \%$ (Fig. 13). Therefore, much of the variability observed in the results from seawater analysis must be attributed to factors other than errors in the calibration of isotope yield monitors.

Numerous sources of ${ }^{232} \mathrm{Th}$ contamination were encountered by participants, including sample containers, reagents, and labware. These sources of contamination were identified and eliminated or reduced (see above as well as accompanying papers in this volume by Andersson and Schöberg 2012; Auro et al. 2012).

Special systems designed to collect samples for the analysis of contamination-prone trace metals are not necessary when sampling seawater to determine concentrations of dissolved ${ }^{232} \mathrm{Th}$, ${ }^{230} \mathrm{Th}$ and ${ }^{231} \mathrm{~Pa}$ (see "Niskin vs. GO-Flo" and Fig. 2). Standard Niskin bottles can be used, although precautions should be taken to minimize chances for contamination during sampling.

Concentrations of dissolved ${ }^{232} \mathrm{Th},{ }^{230} \mathrm{Th}$, and ${ }^{231} \mathrm{~Pa}$ can be determined by filtering with either Nuclepore or Supor membranes (Figs. 4, 5). No significant differences in dissolved concentrations of ${ }^{232} \mathrm{Th},{ }^{230} \mathrm{Th}$, or ${ }^{231} \mathrm{~Pa}$ were detected in using filter membranes with pore diameters ranging between 0.4 and $1.0 \mu \mathrm{m}$. A single test suggested that QMA (quartz) filters may be used as well, although this finding needs verification, especially for ${ }^{231} \mathrm{~Pa}$.

Isotope yield monitors need not be added to samples at sea. There was no detectable loss of dissolved ${ }^{232} \mathrm{Th},{ }^{230} \mathrm{Th}$, or ${ }^{231} \mathrm{~Pa}$ during storage for up to 3 years from seawater that had been filtered and acidified at sea (Fig. 2). Isotope yield monitors equilibrate rapidly with Th dissolved in seawater (too rapidly to establish a rate constant using the data presented here) whereas spike equilibration requires about a day for U (Fig. 3).

Sampling at the BATS site on three occasions over a period of 16 years, by three different labs, produced internally consistent concentration profiles of ${ }^{232} \mathrm{Th}$ and ${ }^{230} \mathrm{Th}$ (Fig. 16). Consistency among these results demonstrates the value of the BATS site as a GEOTRACES baseline station. Other labs can use the relatively unvarying concentrations of ${ }^{232} \mathrm{Th}$ and ${ }^{230} \mathrm{Th}$ at mid-depths for future intercalibration, with reasonable confidence that the concentrations of ${ }^{232} \mathrm{Th}$ and ${ }^{230} \mathrm{Th}$ will be close to those reported in this study. Concentrations in surface and bottom waters may change, however, and should not be used for intercalibration. Results from the Pacific (HOT site) indicate that concentrations of dissolved ${ }^{230} \mathrm{Th}$ may vary by up to $20 \%$ at subannual time scales even at mid-depths (Fig. 17), a possibility that warrants further investigation. 


\section{Recommendations}

Taking into account the lessons learned through the intercalibration initiative, we now consider steps to bring the overall consistency among labs that determine Th and Pa concentrations in seawater from its current best-case level $( \pm \sim 7 \%$, Fig. 9) toward the reproducibility demonstrated during the analysis of SW STD 2010-1 ( $\pm \sim 1 \%$; Fig. 13). First we note contributions to what we have identified as the three principal sources of error (sampling, chemical purification procedures, and instrumental analyses), and then we make specific recommendations for near-term testing and development, as well as general recommendations for procedures to be adopted during the remainder of the GEOTRACES program.

Sampling errors: Three sampling issues were identified during the intercalibration

1) The sensitivity of operationally defined concentrations of dissolved $\mathrm{Th}$ and $\mathrm{Pa}$ to filtration method remains incompletely resolved. Although there was no detectable difference among concentrations of dissolved Th and Pa determined by filtration through membranes of different composition and with pore diameters between 0.4 and 1.0 $\mu \mathrm{m}$ (Figs. 4 and 5), concentrations of Th were significantly lower in samples filtered through a membrane with a pore diameter of $0.22 \mu \mathrm{m}$ (Fig. 4). The extent to which $\mathrm{Th}$ in seawater resides on particles $<0.4 \mu \mathrm{m}$ in diameter should be tested more completely.

2) The possibility of filtration artifacts was also identified. Cartridges used to filter through a $0.22 \mu \mathrm{m}$ pore diameter membrane were much larger (1.6 square meters membrane surface area; > 1 L dead volume) than the individual membrane filters (47 or $90-\mathrm{mm}$ diameter) and Acropak capsules (500 $\mathrm{cm}^{2}$ membrane surface area; $30 \mathrm{~mL}$ dead volume) used to filter other samples. Whether the lower Th concentrations in samples filtered through $0.22 \mu \mathrm{m}$ pore diameter membranes is a filtration artifact, related to sorption of dissolved Th to cartridge and membrane surfaces, or a measure of the true amount of Th associated with particles with an effective diameter between 0.22 and $0.4 \mu \mathrm{m}$, remains unresolved.

Pall Acropak 500 capsule filters, or an equivalent product, were recommended for use on GEOTRACES cruises (see Sampling and Sample-handling Protocols for GEOTRACES Cruises available via the GEOTRACES web site at http://www.geotraces.org/libraries/documents/Intercalibration/Cookbook.pdf) based on their flow rates (resistance to clogging) and the ability to clean them against trace metal contamination. This recommendation was made before the filter tests shown in Figs. 4 and 5 were completed, and the Acropak capsules have never been tested directly against individual (47-mm or $90-\mathrm{mm}$ ) membrane filters or against filters with smaller effective pore diameters for the determination of dissolved $\mathrm{Th}$ and $\mathrm{Pa}$ concentrations. These tests need to be carried out together with further testing of large cartridges, such as the Osmonics cartridge used to fill the SAFe tanks.

3) Contamination of sample containers with Th was evident in a small number of cases during each intercalibration cruise (e.g., Fig. 7 Lab 7; Fig. 9 Lab 9). In some cases the contaminated containers were cleaned by procedures that left no detectable contamination in other containers of the same batch. Whether the observed contamination occurred in the shore-based labs or during sample collection at sea is unknown, but the experience emphasizes the need for precautions against contamination at all stages of sampling and sample handling.

Chemical purification: Both blanks and chemical yields are issues of concern

1) High chemical yields are crucial because, as noted above, the determination of dissolved and particulate $\mathrm{Th}$ and $\mathrm{Pa}$ concentrations in seawater in many cases is near the detection limit for these nuclides. In some cases (not described in this manuscript), participating labs reported low chemical yields. In some cases, the cause of low yields remains unknown, but in one case (Auro et al. 2012), it was traced to the irreversible sorption of Th by material used in the construction of ion exchange columns. Investigators are encouraged to report the cause of low yields when they are identified.

2) Reagent blanks can be large. As noted above, high purity iron carrier may contain large amounts of Th. Ion exchange resin (e.g., Auro et al. 2012) can also be a source of substantial Th contamination. Contamination can vary by a large amount from one batch of reagents to another. Each batch of reagents should be tested carefully and processed if possible (e.g., for iron carrier or for ion exchange resin) to reduce blanks to tolerable levels.

3) Isobaric interferences from molecular species were not discussed in this article, but they are a concern when measuring $\mathrm{Th}$ and $\mathrm{Pa}$ by mass spectrometry. Careful purification of Th and $\mathrm{Pa}$ will help reduce isobaric interferences.

\section{Instrumentation}

The three principal types of mass spectrometers used to measure concentrations of $\mathrm{Th}$ and $\mathrm{Pa}$ were identified in the Introduction. Some issues are unique to one type of instrument while other issues are common to all.

1) Filament blanks, applicable to TIMS, are contributed from the rhenium filament as well as from colloidal graphite applied to facilitate ionization of Th (e.g., see above as well as Andersson and Schöberg 2012; Edwards et al. 1987). The procedure followed at the University of Minnesota for quality control of filaments is described in "Mass spectrometry" as an example that may be followed by other labs.

2) Mass bias corrections can be large during analyses by ICPMS. For example, SRM 129 with a natural ${ }^{238} \mathrm{U} /{ }^{235} \mathrm{U}$ abundance of 137.88 is used at LDEO to determine mass bias. Measured 238/235 ratios accompanying seawater analyses 
at LDEO varied between 138 and 141. Mass bias must be measured frequently and incorporated into data reduction routines. It is generally assumed that mass bias for $\mathrm{U}$ is not significantly different from that for $\mathrm{Th}$ and $\mathrm{Pa}$, although most labs have not tested this assumption on their own instruments.

3) Isobaric interferences, mentioned above, can be controlled to some extent during ICP-MS analysis by membrane desolvation and by adjusting instrument settings (e.g., Choi et al. 2001). Investigators should take into account isobaric interferences as well as sensitivity, abundance sensitivity, and other factors when optimizing each instrument.

4) Variable gain among multiple detectors adds to the uncertainty in measured atom ratios. This includes the variability in gain between an ion counter and a Faraday cup as well as variability between multiple ion counters.

5) The linearity of detector response as a function of absolute signal intensity was generally assumed by participating labs not to be an issue, but this is a potential source of uncertainty that should be considered in future studies.

Many of the items listed above may have contributed to the observed variability of ${ }^{232} \mathrm{Th} /{ }^{229} \mathrm{Th}$ ratios during the analysis of SW STD 2010-2 (Fig. 14). Each of these topics warrants further examination as the community measuring concentrations of $\mathrm{Th}$ and $\mathrm{Pa}$ in seawater works toward improving internal consistency.

\section{General recommendations}

The following recommendations expand upon the specific issues identified above. They were developed in the context of improving results from future GEOTRACES cruises although they apply to other sampling programs as well.

1) Procedural blanks should be collected frequently at sea; for example, one seawater blank is collected at each station on U.S. GEOTRACES cruises by adding 2 to 3 L Milli-Q (or equivalent) water to a sample container and processing it as a sample. Improved characterization of blanks will better constrain overall uncertainties, even if the blanks themselves cannot be reduced significantly. High blanks should only affect detection limits, not accuracy, if the blank is well characterized statistically.

2) Concentrations of dissolved ${ }^{230} \mathrm{Th}$ and ${ }^{231} \mathrm{~Pa}$ in $\sim 10 \mathrm{~L}$ surface ocean water are close to the detection limits for many labs. Analysis of replicate samples from surface waters (the mixed layer) will improve the reliability of results.

3) Concentrations of particulate ${ }^{230} \mathrm{Th}$ and ${ }^{231} \mathrm{~Pa}$ in $\sim 100 \mathrm{~L}$ seawater are below the limit of detection for many labs. It is recommended that the community work together to either lower detection limits or increase sample sizes for particulate material. Alternatively, it may be necessary to designate a subset of labs with the best detection limits to analyze samples of particulate material for ${ }^{230} \mathrm{Th}$ and ${ }^{231} \mathrm{~Pa}$ collected during GEOTRACES cruises.

4) The distribution and analysis of the artificial seawater solutions (SW STD 2010-1 and 2010-2) helped constrain the internal consistency among the labs reporting results (Fig. 13). It is recommended that these solutions, or an equivalent, be analyzed routinely when determining the concentrations of dissolved ${ }^{230} \mathrm{Th},{ }^{232} \mathrm{Th}$, and ${ }^{231} \mathrm{~Pa}$ in seawater samples, much like the SAFe seawater standards that are analyzed routinely during the determination of trace metal concentrations in seawater (http://www. geotraces.org/science/intercalibration/322-standards-andreference-materials).

5) Results from the SW STD solutions (Figs. 13 and 14) also illustrate the large differences in the magnitude of the errors reported among participating labs. This difference is due in part to the different instruments used to measure atom ratios, and the different instrumental procedures (e.g., the number of atom ratios averaged), but it also reflects the differences among participating labs in the protocols used to evaluate and report errors. It was beyond the scope of this intercalibration to make a synthesis and evaluation of the different error reporting protocols, but a comparison of two of the participating labs illustrates the nature of the difference. As noted above, investigators at LDEO propagate one sigma errors in atom ratios measured by ICP-MS with uncertainties in background count rate, tail corrections, and spike calibration to report their analytical uncertainty. By contrast, investigators at WHOI propagate two standard errors (two sigma divided by the square root of the number of ratios) of the atom ratios determined by ICP-MS with uncertainties in background count rate and spike calibration to report analytical uncertainty (Auro et al. 2012). We recommend that a comparison be made of the methods used to report uncertainties and that the results of that synthesis be reported in a future publication.

6) A standard protocol for GEOTRACES cruises is to collect replicate samples to be analyzed by a lab other than the main lab(s) participating in the cruise. Although this recommendation is directed primarily to labs that have not participated in the GEOTRACES intercalibration exercise, it is good general advice under all circumstances. Having a second lab analyze replicate samples will identify inconsistencies and assure overall data quality. The authors recommend that replicate samples be collected for analysis by a collaborating lab as a standard protocol for future work on dissolved ${ }^{230} \mathrm{Th},{ }^{232} \mathrm{Th}$, and ${ }^{231} \mathrm{~Pa}$ in seawater.

\section{Acknowledgments}

The GEOTRACES intercalibration initiative has proven to be a valuable asset for the marine geochemistry community. It owes much of its success to the tireless efforts of Greg Cutter, who led the initiative from the initial planning through cruise coordination to the completion of this special volume. The intercalibration results described in this manuscript would not have been possible without contributions from many individuals. Katharina Pahnke, Tina van de Flierdt, Dennis Graham, 
and Tim Kenna helped with sample collection at sea. Chris Hayes helped track down sources of ${ }^{232} \mathrm{Th}$ blanks at LDEO. Geoffrey Smith designed and constructed the SAFe tank system, and he was responsible for filtering and acidifying the homogenized seawater that was sampled for distribution to the labs participating in this intercalibration. Samples of particulate material collected by in situ filtration were kindly provided by Ken Buesseler, Kanchan Maiti, and Steve Pike. Sample analysis, data reduction, and comments on the manuscript and/or on intercalibration procedures were generously contributed by Ayako Okubo, Zanna Chase, Celia Venchiarutti, Per Andersson, Hans Schöberg, Abel Guihou, Sylvain Pichat, Gi-Hoon Hong, Maureen Auro, Louisa Bradtmiller, Gideon Henderson, Hai Cheng, Xianfeng Wang, Walter Geibert, Catherine Jeandel, Franco Marcantonio, Hisao Nagai, Jan Scholten, François Thil, Esther Le Toquin, and Masatoshi Yamada. Guest editor Peter Croot and four anonymous referees are thanked for their constructive comments and recommendations. Funding for sample collection and for the coordination of this intercalibration of Th isotopes, ${ }^{231} \mathrm{~Pa}$ and ${ }^{10} \mathrm{Be}$ was provided by the U.S. NSF (to Anderson, Moran, and Edwards). Funding from NSF (to R. Francois and to K. Buesseler) also supported the collection of previously unpublished data that are presented here. This paper is part of the Intercalibration in Chemical Oceanography special issue of L\&O Methods that was supported by funding from the U.S. National Science Foundation, Chemical Oceanography Program (Grant OCE- 0927285 to G. Cutter).

\section{References}

Anderson, R. F., M. P. Bacon, and P. G. Brewer. 1983a. Removal of ${ }^{230} \mathrm{Th}$ and ${ }^{231} \mathrm{~Pa}$ at ocean margins. Earth Planet. Sci. Lett. 66:73-90 [doi:10.1016/0012-821X(83)90127-9].

- M. P. Bacon, and P. G. Brewer. 1983b. Removal of ${ }^{230} \mathrm{Th}$ and ${ }^{231} \mathrm{~Pa}$ from the open ocean. Earth Planet. Sci. Lett. 62:723 [doi:10.1016/0012-821X(83)90067-5].

Andersson, P. S., and H. Schöberg. In press. Determination of ${ }^{232} \mathrm{Th}$ and ${ }^{230} \mathrm{Th}$ in seawater using a chemical separation procedure and thermal ionization mass spectrometry. Limnol. Oceanogr. Methods.

Auro, M., L. F. Robinson, A. Burke, L. I. Bradtmiller, M. Q. Fleisher, and R. F. Anderson. In press. Intercal: Analysis of 232-thorium, 230-thorium and 231-protactinium in seawater: Lessons learned during GEOTRACES Intercalibration. Limnol. Oceanogr. Methods.

Bacon, M. P., and R. F. Anderson. 1982. Distribution of thorium isotopes between dissolved and particulate forms in the deep-sea. J. Geophys. Res. Oceans Atmos. 87:2045-2056 [doi:10.1029/JC087iC03p02045].

Chase, Z., R. F. Anderson, M. Q. Fleisher, and P. Kubik. 2002. The influence of particle composition on scavenging of Th, $\mathrm{Pa}$ and Be in the ocean. Earth Planet. Sci. Lett. 204:215-229 [doi:10.1016/S0012-821X(02)00984-6].

$\longrightarrow,-$, $\longrightarrow$, 2003. Scavenging of ${ }^{230} \mathrm{Th}$,
${ }^{231} \mathrm{~Pa}$, and ${ }^{10} \mathrm{Be}$ in the Southern Ocean (SW Pacific sector): The importance of particle flux and advection. Deep-Sea Res. II 50:739-768 [doi:10.1016/S0967-0645(02)00593-3].

Chen, J. H., R. L. Edwards, and G. J. Wasserburg. 1986. ${ }^{238} \mathrm{U}$, ${ }^{234} \mathrm{U}$ and ${ }^{232} \mathrm{Th}$ in seawater. Earth Planet. Sci. Lett. 80:241251 [doi:10.1016/0012-821X(86)90108-1].

Choi, M. S., and others. 2001. Rapid determination of ${ }^{230} \mathrm{Th}$ and ${ }^{231} \mathrm{~Pa}$ in seawater by desolvated micro-nebulization Inductively Coupled Plasma magnetic sector mass spectrometry. Mar. Chem. 76:99-112 [doi:/10.1016/S03044203(01)00050-0].

Cutter, G. A., and K. W. Bruland. In press. Rapid and non-contaminating sampling system for trace elements in global ocean surveys. Limnol. Oceanogr. Methods.

De Baar, H. J. W., and others. 2008. Titan: A new facility for ultraclean sampling of trace elements and isotopes in the deep oceans in the international Geotraces program. Mar. Chem. 111:4-21 [doi:10.1016/j.marchem.2007.07.009].

Edwards, R. L., J. H. Chen, and G. J. Wasserburg. 1987. ${ }^{238} \mathrm{U}-$ ${ }^{234} \mathrm{U}-230 \mathrm{Th}-{ }^{232} \mathrm{Th}$ systematics and the precise measurement of time over the past 500000 years. Earth Planet. Sci. Lett. 81:175-192 [doi:/10.1016/0012-821X(87)90154-3].

Fleisher, M. Q., and R. F. Anderson. 2003. Assessing the collection efficiency of Ross Sea sediment traps using ${ }^{230} \mathrm{Th}$ and ${ }^{231} \mathrm{~Pa}$. Deep-Sea Res. II 50:693-712 [doi:10.1016/S09670645(02)00591-X].

Francois, R. 2007. Paleoflux and paleocirculation from Sediment ${ }^{230} \mathrm{Th}$ and ${ }^{231} \mathrm{~Pa} /{ }^{230} \mathrm{Th}$, p. 681-716. In C. Hillaire-Marcel and A. de Vernal [eds.], Proxies in late cenozoic paleoceanography. Elsevier.

Goldberg, E. D., R. H. Smith, M. Koide, and R. A. Schmitt. 1963. Rare-earth distributions in the marine environment. J. Geophys. Res. 68:4209-4217.

Henderson, G. M., and R. F. Anderson. 2003. The U-series toolbox for paleoceanography. Rev. Mineral. Geochem. 52:493529 [doi:10.2113/0520493].

Huh, C. A., and T. M. Beasley. 1987. Profiles of dissolved and particulate thorium isotopes in the water column of coastal Southern-California. Earth Planet. Sci. Lett. 85:1-10 [doi:10.1016/0012-821X(87)90016-1].

Luo, Y., R. Francois, and S. E. Allen. 2010. Sediment ${ }^{231} \mathrm{~Pa} /{ }^{230} \mathrm{Th}$ as a recorder of the rate of the Atlantic meridional overturning circulation: insights from a 2-D model. Ocean Sci. 6:381-400 [doi:10.5194/os-6-381-2010].

Maiti, K., and others. In press. Intercalibration studies of short-lived Thorium-234 in the water column and marine particles. Limnol. Oceanogr. Methods.

Moore, W. S., and W. M. Sackett. 1964. Uranium and thorium series inequilibrium in seawater. J. Geophys. Res. 69:54015405 [doi:10.1029/JZ069i024p05401].

Moran, S. B., M. A. Charette, J. A. Hoff, R. L. Edwards, and W. M. Landing. 1997. Distribution of ${ }^{230} \mathrm{Th}$ in the Labrador Sea and its relation to ventilation. Earth Planet. Sci. Lett. 150:151-160 [doi:10.1016/S0012-821X(97)00081-2]. 
and others. 2001. Constraints on deep water age and particle flux in the Equatorial and South Atlantic Ocean based on seawater ${ }^{231} \mathrm{~Pa}$ and ${ }^{230} \mathrm{Th}$ data. Geophys. Res. Lett. 28:3437-3440.

, C. C. Shen, H. N. Edmonds, S. E. Weinstein, J. N. Smith, and R. L. Edwards. 2002. Dissolved and particulate ${ }^{231} \mathrm{~Pa}$ and ${ }^{230} \mathrm{Th}$ in the Atlantic Ocean: constraints on intermediate/deep water age, boundary scavenging, and ${ }^{231} \mathrm{~Pa} /{ }^{230} \mathrm{Th}$ fractionation. Earth Planet. Sci. Lett. 203:9991014 [doi:10.1016/S0012-821X(02)00928-7].

Nozaki, Y., Y. Horibe, and H. Tsubota. 1981. The water column distribution of thorium isotopes in the western North Pacific. Earth Planet. Sci. Lett. 54:203-216 [doi:10.1016/ 0012-821X(81)90004-2].

, and Y. Horibe. 1983. Alpha-emitting thorium isotopes in northwest Pacific deep waters. Earth Planet. Sci. Lett. 65:39-50 [doi:10.1016/0012-821X(83)90188-7].

—, and T. Nakanishi. 1985. ${ }^{231} \mathrm{~Pa}$ and ${ }^{230} \mathrm{Th}$ profiles in the open ocean water column. Deep-Sea Res. A 32:1209-1220.

Owens, S. A., K. O. Buesseler, and K. W. W. Sims. 2011. Re-evaluating the ${ }^{238} \mathrm{U}$-salinity relationship in seawater: Implications for the ${ }^{238} \mathrm{U}-{ }^{234} \mathrm{Th}$ disequilibrium method. Mar. Chem. 127:31-39 [doi:10.1016/j.marchem.2011.07.005].

Robinson, L. F., N. S. Belshaw, and G. M. Henderson. 2004. U and Th concentrations and isotope ratios in modern carbonates and waters from the Bahamas. Geochim. Cos- mochim. Acta 68:1777-1789 [doi:10.1016/j.gca.2003.10.005]. , J. H. Chen, and G. J. Wasserburg. 1996. ${ }^{230} \mathrm{Th}-{ }^{232}$ Th systematics in the central Pacific Ocean: The sources and the fates of thorium. Earth Planet. Sci. Lett. 139:351-363 [doi:10.1016/0012-821X(96)00017-9].

Rutgers Van Der Loeff, M. M., and W. Geibert. 2008. U- and Th-series nuclides as tracers of particle dynamics, scavenging and biogeochemical cycles in the oceans, p. 227-268. In S. Krishnaswami and J. K. Cochran [eds.], U/Th series radionuclides in aquatic systems. Radioactivity in the environment. Elsevier.

Shen, C. C., and others. 2002. Uranium and thorium isotopic and concentration measurements by magnetic sector inductively coupled plasma mass spectrometry. Chem. Geol. 185:165-178 [doi:10.1016/S0009-2541(01)00404-1].

, and others. 2003. Measurement of attogram quantities of ${ }^{231} \mathrm{~Pa}$ in dissolved and particulate fractions of seawater by isotope dilution thermal ionization mass spectroscopy. Anal. Chem. 75:1075-1079 [doi:10.1021/ac026247r].

Wei, C. L., and C. C. Hung. 1993. The effect of isotopic equilibration time on the determination of ${ }^{234} \mathrm{Th}$ in seawater. J. Radioanal. Nucl. Chem. Lett. 175:155-159 [doi:10.1007/ BF02164114].

Submitted 7 August 2011 Revised 7 February 2012 Accepted 14 February 2012 